\title{
Non-Directed, Copper Catalyzed Benzylic C-H Amination Avoiding Sub- strate Excess
}

\author{
Anqi Wang, ${ }^{\dagger}$ Cristina C. DeOliveira, ${ }^{\dagger}$ and Marion H. Emmert ${ }^{\dagger *}$ \\ †Department of Chemistry and Biochemistry, Worcester Polytechnic Institute, 100 Institute Road, Worcester, MA 01609, \\ U.S.A. \\ †Current Address: Process Research \& Development, MRL, Merck \& Co. Inc, 770 Sumneytown Pike, West Point, PA, \\ 19486, U.S.A.
}

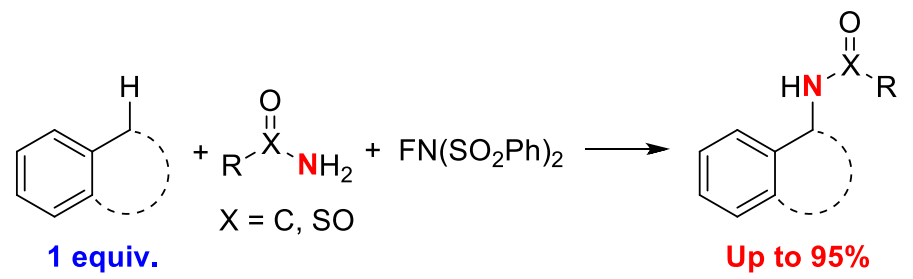

\begin{abstract}
We report the development of a benzylic C-H amination protocol that addresses two common drawbacks in nondirected, intermolecular benzylic C-H aminations: (i) the need to use an excess of substrate and (ii) the limitation to only introduce one type of nitrogen source. Key to this discovery is the use of the strong oxidant $N$-fluorobenzenesulfonimide (NFSI) in combination with a $\mathrm{Cu}$ /diimine ligand catalyst system. The established conditions allow to lower the $\mathrm{C}-\mathrm{H}$ substrate loading to 1.0 equivalent and provide up to $95 \%$ yield of $\mathrm{C}-\mathrm{H}$ amination product. Furthermore, sulfonamides and benzamides can be employed as nitrogen sources, resulting in access to a diverse product scope.
\end{abstract}

Efficiently converting $\mathrm{C}-\mathrm{H}$ bonds into other functional groups in organic molecules has been of prime interest to the synthetic community in the last decades. ${ }^{1-6}$ The most prominent approach to achieve this goal, so-called directed or chelate-assisted $\mathrm{C}-\mathrm{H}$ functionalization, has established the use of directing groups that lead a catalyst to a specific $\mathrm{C}-\mathrm{H}$ bond. ${ }^{7-9}$ This directed $\mathrm{C}-\mathrm{H}$ bond attack provides access to many sites within complex targets that are otherwise difficult to modify at late stages of a synthesis.

In comparison to the vast knowledge that has been created for these directed reactions, non-chelate-assisted C-H functionalizations are underdeveloped. ${ }^{10,11}$ This is likely due to several fundamental barriers to access such reactivity: (i) The non-directed cleavage of $\mathrm{C}-\mathrm{H}$ bonds is often kinetically challenging. Therefore, such transformations frequently require an excess of substrate, making them less synthetically useful. (ii) Due to the significant strength and high $\mathrm{pK}_{\mathrm{a}}$ 's of $\mathrm{C}-\mathrm{H}$ bonds, many reagents used in oxidative $\mathrm{C}-\mathrm{H}$ functionalizations are highly reactive and can thus lead to side reactions. In combination with slow $\mathrm{C}-\mathrm{H}$ bond cleavage, this can cause low selectivities, therefore also favoring conditions with high substrate loadings.

Due to these challenges, it is not surprising that many benzylic C-H amination protocols that do not proceed through chelate-assistance require an excess of substrate. ${ }^{12-17}$ Among those examples is a recent protocol developed in our group that employs a $\mathrm{Cu}$-based catalyst system and protected hydroxylamines as reagents. ${ }^{18}$ Based on detailed mechanistic studies, we speculated that departure from the hydroxylamine scaffold towards a combination of oxidant and $\mathrm{N}$ source should be effective with the same catalyst system. Examples of such systems have been reported by Warren and coworkers, using ${ }^{t} \mathrm{BuOO}^{t} \mathrm{Bu}$ as oxidant. ${ }^{13,15}$ We were further inspired by an example of benzylic C-H cyanation employing $N$-fluorobenzenesulfonimide (NFSI) published by Stahl, Liu, and co-workers. ${ }^{19}$ NFSI has further been employed as oxidant and $\mathrm{N}$ source in a $\mathrm{Cu}$ catalyzed $\mathrm{C}-\mathrm{H}$ amination that is selective for primary benzylic $\mathrm{C}-\mathrm{H}$ bonds and introduces the $-\mathrm{N}\left(\mathrm{SO}_{2} \mathrm{PH}\right)_{2}$ group (Scheme $\left.1 \mathrm{~B}\right){ }^{20}$

A. ${ }^{t} \mathrm{BuOO}^{t} \mathrm{Bu}$ as Oxidant

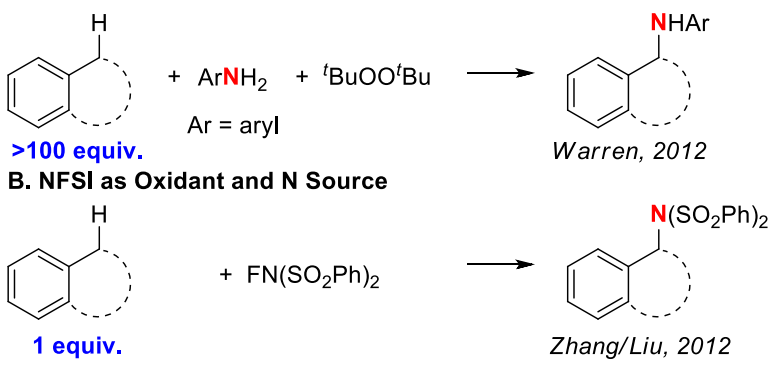

C. This Work: NFSI as Oxidant \& Variable N Source

1 equiv.

Up to $95 \%$

Scheme 1. Previous and herein reported Cu catalyzed, benzylic, non-directed $\mathrm{C}-\mathrm{H}$ aminations. 
As the latter example is strongly limited with regards to synthetic applications, we reasoned that the introduction of an external nucleophile in combination with NFSI may provide variability for the direct synthesis of protected benzylic amines (Scheme 1C). This manuscript describes the development and scope of the resulting methodology.

Optimizations with 1 equivalent of ethylbenzene as substrate (for all details, see the SI) led to the identification of conditions, which afforded the amination product $\mathrm{PhCHNHTsCH}_{3}$ in excellent yield (95\%; Table 1, entry 1). In contrast to the prior reactivity established with NFSI (Scheme 1B) ${ }^{20}$ it was crucial to perform the reaction under a nitrogen atmosphere; the presence of air resulted in a significantly diminished yield $(64 \%$; entry 2). Both the $\mathrm{Cu}$ catalyst and the oxidant were required for reactivity, as illustrated by $0 \%$ yield in their absences (entries 3 and 4). Interestingly, only slightly diminished loadings of NFSI or $\mathrm{TsNH}_{2}$ (2 equiv.) resulted in noticeably lower yields $(68 \%$ and $63 \%$, respectively; entries 5 and 6).

Table 1. Selected Optimization and Background Studies.

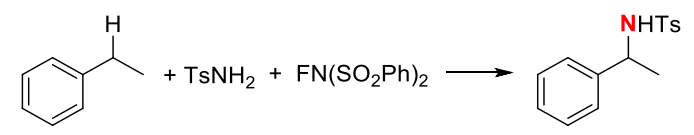

\begin{tabular}{lll}
\hline Entry & Changes to Standard Conditions ${ }^{\mathrm{a}}$ & Yield $^{\mathrm{b}}$ \\
\hline $\mathbf{1}$ & None & $\mathbf{9 5 \%}$ \\
2 & air atmosphere & $64 \%$ \\
3 & no Cu catalyst precursor & $0 \%$ \\
4 & No NFSI & $0 \%$ \\
5 & 2 equiv. NFSI & $68 \%$ \\
6 & 2 equiv. TsNH 2 & $63 \%$ \\
7 & MeCN as solvent & $57 \%$ \\
8 & C6 6 6 as solvent & $73 \%$ \\
9 & 1,2-dichloroethane as solvent & $92 \%$ \\
10 & Cu(OTf)2 instead of Cu(BF $)_{2} \cdot 6 \mathrm{H}_{2} \mathrm{O}$ & $7 \%$ \\
11 & Ligand $\mathbf{2}$ instead of $\mathbf{1}$ & $72 \%$ \\
12 & Ligand $\mathbf{3}$ instead of $\mathbf{1}$ & $76 \%$ \\
13 & Ligand $\mathbf{4}$ instead of $\mathbf{1}$ & $72 \%$ \\
14 & Ligand $\mathbf{5}$ instead of $\mathbf{1}$ & $57 \%$ \\
15 & Ligand $\mathbf{6}$ instead of $\mathbf{1}$ & $32 \%$ \\
16 & Ligand $\mathbf{7}$ instead of $\mathbf{1}$ & $62 \%$ \\
17 & Ligand $\mathbf{8}$ instead of $\mathbf{1}$ & $69 \%$ \\
18 & Ligand $\mathbf{9}$ instead of $\mathbf{1}$ & $82 \%$ \\
19 & No ligand & $75 \%$ \\
\hline
\end{tabular}

aStandard Conditions: $\mathrm{Cu}\left(\mathrm{BF}_{4}\right)_{2} \cdot 6 \mathrm{H}_{2} \mathrm{O}(4.6 \mathrm{mg}, 12.6 \mu \mathrm{mol}$, $10.0 \mathrm{~mol} \%)$, ligand 1 (6.0 mg, $12.6 \mu \mathrm{mol}, 10.0 \mathrm{~mol} \%), \mathrm{TsNH}_{2}$ (64.2 mg, 0.375 mmol, 3.00 equiv.), NFSI (118.3 mg, 0.375 mmol, 3.00 equiv.), ethylbenzene (16.0 $\mu \mathrm{L}, 13.3 \mathrm{mg}, 0.125$ mmol, 1.00 equiv.), $\mathrm{PhCF}_{3}(1.5 \mathrm{~mL}), 110{ }^{\circ} \mathrm{C}, 48 \mathrm{~h}, \mathrm{~N}_{2} .{ }^{\text {b }}$ Yields were determined by quantitative, crude ${ }^{1} \mathrm{H}$ NMR using $\mathrm{ClH}_{2} \mathrm{CCHCl}_{2}$ as internal standard. All yields are shown as averages of at least two catalytic runs.
Similarly, use of different solvents than trifluorotoluene also resulted in diminished yields (entries 7 to 9), with 1,2-dichloroethane performing best among the alternative solvents $(92 \%)$. Furthermore, the identity of the $\mathrm{Cu}$ catalyst precursor was shown to be important: Using $\mathrm{Cu}(\mathrm{OTf})_{2}$ instead of $\mathrm{Cu}\left(\mathrm{BF}_{4}\right)_{2} \cdot 6 \mathrm{H}_{2} \mathrm{O}$ reduced the product yield to only $7 \%$ (Table 1 , entry 10$)$.

Interestingly, $\mathrm{C}-\mathrm{H}$ amination reactivity was observed regardless of the identity of the used diimine ligand: Employing the electron-deficient ligands $\mathbf{2}, \mathbf{3}$, and $\mathbf{4}$ (Scheme 2) afforded 72 to $76 \%$ yield (entries 11 to 13 ). Using the $\mathrm{NO}_{2}$-substituted ligand $\mathbf{5}$, which has shown high activity in combination with $\mathrm{Cu}$ catalysts and Chloramine-T as oxidant, ${ }^{21}$ afforded only $57 \%$ yield (entry 14), while an electron-rich diimine ligand (6) resulted in even lower yield (32\%; entry 15) than the reaction in the absence of any added ligand (75\%, entry 19). These observations suggest that a relatively electron-poor $\mathrm{Cu}$ species is important to promote reactivity. In agreement with this hypothesis, electron-rich ligands $\mathbf{7}$ and $\mathbf{8}$ that are frequently used in the literature in combination with $\mathrm{Cu}$ catalyst precursors provide mediocre yields in $\mathrm{C}-\mathrm{H}$ amination $(62 \%$ and $69 \%$, respectively; entries $16 / 17)$. Interestingly, the BINAP-derivative 9 is the only other tested ligand than 1 that affords higher yields (82\%; entry 18) than the reaction in the absence of ligand.

Finally, a comparison of product yields at difference temperatures (Scheme 3) further suggests that the highest $\mathrm{C}-\mathrm{H}$ amination reactivities are obtained above $100{ }^{\circ} \mathrm{C}$.
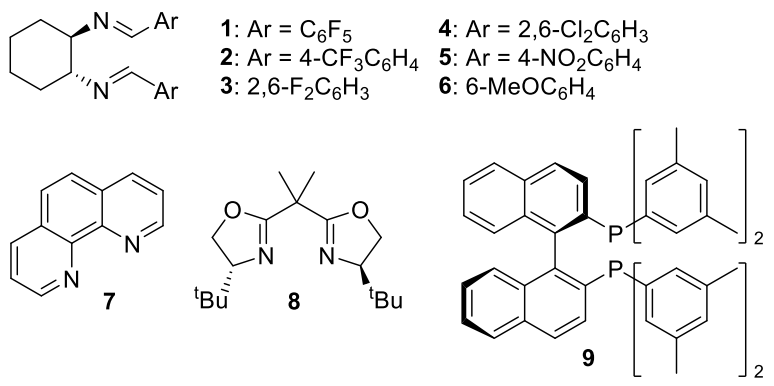

Scheme 2. Ligands Used in Reaction Optimization.
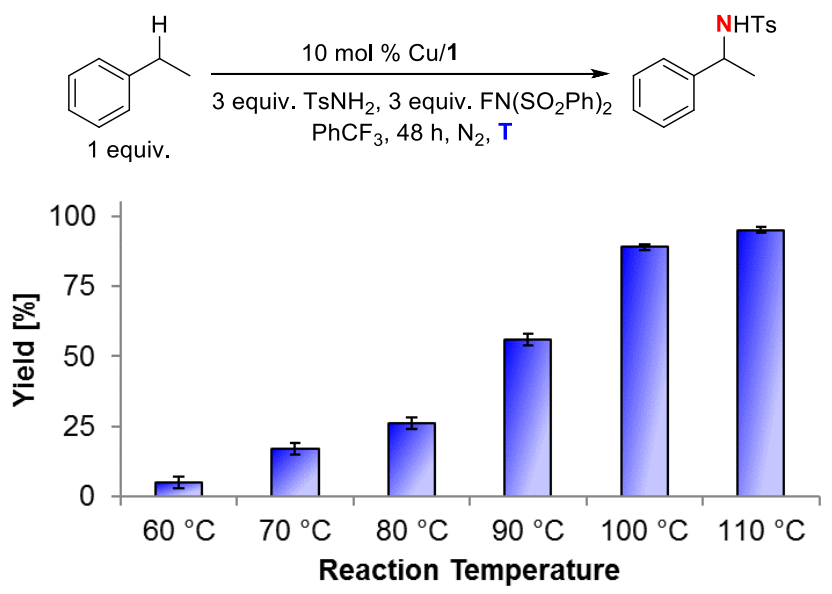

Scheme 3. Temperature Study. 
With the optimized conditions in hand, we turned our attention to exploring the scope of alkyl arenes (Scheme 4). Substrates with only primary or tertiary benzylic $\mathrm{C}-\mathrm{H}$ bonds provided lower reactivity: The $\mathrm{C}-\mathrm{H}$ amination of toluene only afforded 19\% of $\mathrm{C}-\mathrm{H}$ amination product (together with $24 \%$ of $\left.\mathrm{PhCH}_{2} \mathrm{~N}\left(\mathrm{SO}_{2} \mathrm{Ph}\right)_{2}\right)$, while no product was observed in reactions employing cumene as the substrate. $\mathrm{C}-\mathrm{H}$ amination of isobutyl benzene only proceeded in $20 \%$ yield. The latter observations with cumene and isobutyl benzene as substrate imply that the observed reactivity is not only governed by free radical stabilities but is also influenced by the sterics in the immediate surrounding of the benzylic $\mathrm{C}-\mathrm{H}$ bond.

$n$-Propyl benzene and $n$-butyl benzene both afforded mediocre yields of product (47\% and $39 \%$, respectively). Interestingly, 1-ethyl naphthalene showed much higher reactivity (61\%) than 2-ethyl naphthalene (29\%), which may be attributable to the different stabilities of the respective radical intermediates. Diphenylmethane underwent $\mathrm{C}-\mathrm{H}$ amination in high yield $(84 \%)$, further pointing towards the importance of stabilizing potential radical intermediates.

Finally, electron-withdrawing and electron-neutral substituents $\left(\mathrm{F}, \mathrm{Cl}, \mathrm{CF}_{3}\right)$ on the ethyl benzene core were tolerated, resulting in $93 \%, 73 \%$, and $39 \%$ yield of $\mathrm{C}-\mathrm{H}$ amination product. In contrast, 4-methoxyethylbenzene did not afford any distinguishable product under the standard reaction conditions. Generally, the isolated yields (shown in brackets in Scheme 4) are in the same range as the crude yields, but occasionally $\sim 20 \%$ lower due to challenges separating the product from $\left(\mathrm{PhSO}_{2}\right)_{2} \mathrm{NH}$, the side product of oxidation.

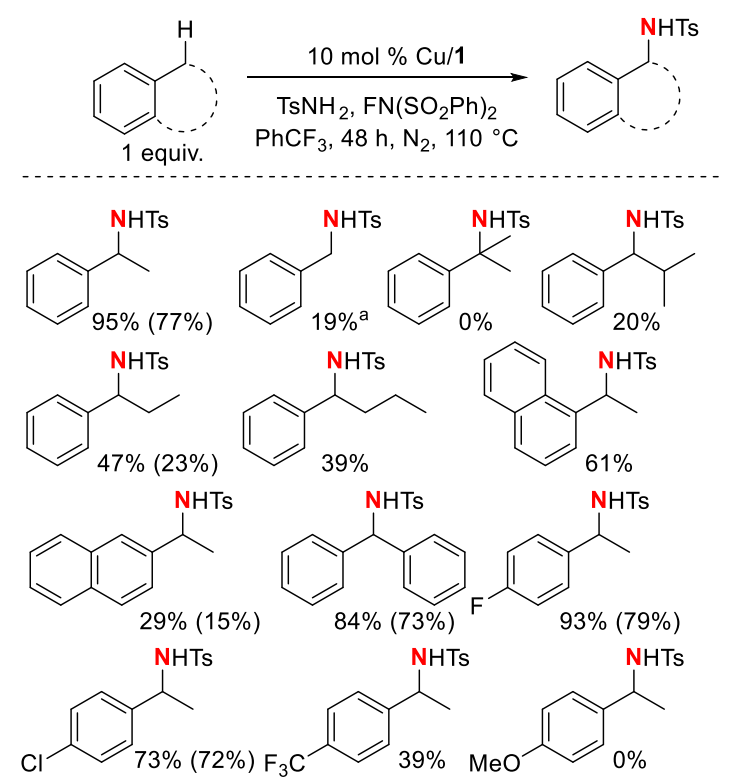

Scheme 4. Benzylic CH Substrate Scope. Yields were determined by quantitative, crude ${ }^{1} \mathrm{H} \mathrm{NMR}$ using $\mathrm{ClH}_{2} \mathrm{CCHCl}_{2}$ as internal standard. Yields are shown as averages of at least two catalytic runs. Isolated yields are shown in brackets. ${ }^{2} 24 \%$ of $\mathrm{PhCH}_{2} \mathrm{~N}\left(\mathrm{SO}_{2} \mathrm{Ph}\right)_{2}$ were also formed.

Motivated by the variability of yields, we focused on gaining a better understanding of limiting factors in the reaction. We thus performed a series of experiments geared at elucidating potential catalyst decomposition (Scheme 5). 1-Ethyl naphthalene was chosen as substrate, due to the mediocre (61\%) yields obtained under standard reaction conditions. To test for catalyst decomposition, we added another batch of catalyst into the mixture after $48 \mathrm{~h}$, followed by another $48 \mathrm{~h}$ of reaction time. Similarly, oxidant decomposition was tested by charging with a second batch of oxidant.

Surprisingly, both reactions afforded significantly lower yields $(22 \%$ and $8 \%$, respectively) than the original $61 \%$ after $48 \mathrm{~h}$; recharging both catalyst and NFSI resulted in $14 \%$ yield. These data imply that the product of $\mathrm{C}-\mathrm{H}$ amination is not stable under the standard reaction conditions. To test if product decomposition is a limiting factor, we subjected $\mathrm{PhCHNHTsCH}_{3}$ to standard reaction conditions (Scheme 6). Only $74 \%$ of $\mathrm{PhCHNHTsCH}_{3}$ were remaining after $48 \mathrm{~h}$, supporting that product decomposition occurs $110^{\circ} \mathrm{C}$. Gratifyingly, no decomposition was observed when the reaction was performed at 90 ${ }^{\circ} \mathrm{C}$ (>99\% $\mathrm{PhCHNHTsCH}_{3}$ after $\left.48 \mathrm{~h}\right)$.

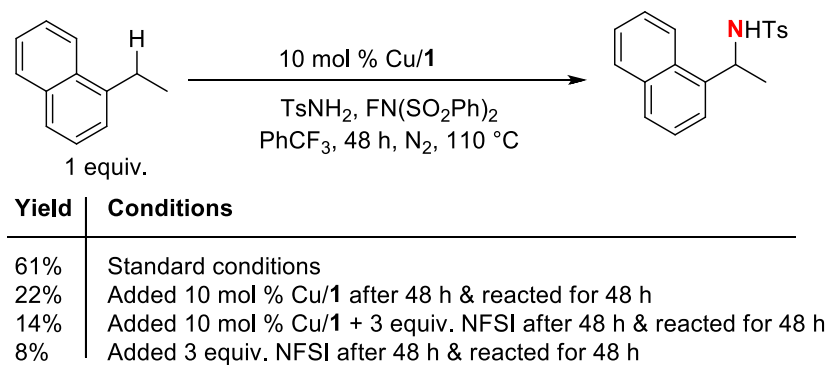

Scheme 5. Test for Catalyst or Oxidant Decomposition.

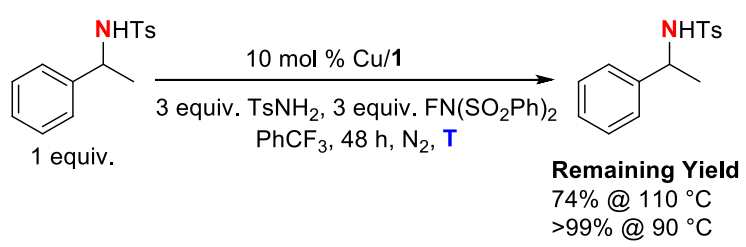

Scheme 6. Product Decomposition Study.

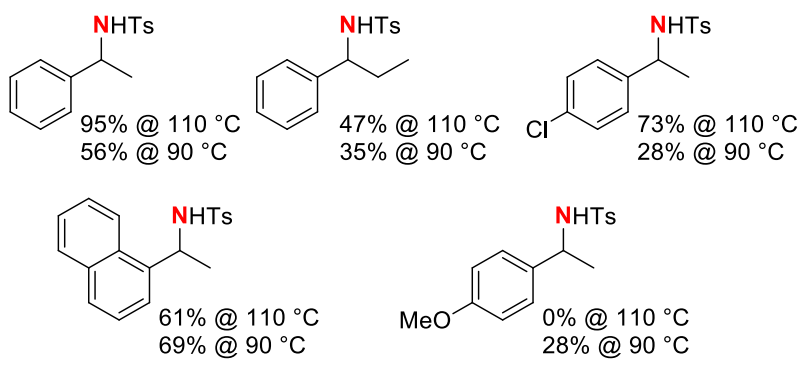

Scheme 7. Comparison of Selected Product Yields at 90 and $110{ }^{\circ} \mathrm{C}$.

Encouraged by this result, we performed C-H aminations of several benzylic substrates at $90{ }^{\circ} \mathrm{C}$; all chosen substrates had not shown quantitative yields under standard conditions (Scheme 7). The obtained yields suggest that product decomposition does not seem to be a major factor in limiting yields for most simple alkylbenzenes (ethyl benzene, n-propyl benzene, 
and 4-chloroethyl benzene shown in Scheme 7; for more examples, see the SI); all these substrates showed decreased yields at $90{ }^{\circ} \mathrm{C}$. In contrast, 1-ethyl naphthalene showed improved yields under these conditions (69\% vs. 61\%). Most excitingly, the yield with 4-methoxyethyl benzene was raised from $0 \%$ to $28 \%$ by lowering the temperature to $90{ }^{\circ} \mathrm{C}$. This suggests that $\mathrm{C}-\mathrm{H}$ amination occurs with 4 -methoxyethyl benzene at $110^{\circ} \mathrm{C}$, but that the product is unstable under standard reaction conditions and thus not observed.

We then investigated if different reaction temperatures influence the site selectivity of $\mathrm{C}-\mathrm{H}$ amination in addition to influencing product stability. To this end, we subjected 4-ethyltoluene to reaction conditions at $110{ }^{\circ} \mathrm{C}$ and at $90{ }^{\circ} \mathrm{C}$ (Scheme $\left.8 \mathrm{~A}\right)$. The reaction at the lower temperature provided both higher product yields (73\% vs. 52\%) and higher selectivity (1:4 vs. 1:3) compared to the reaction outcome at $110^{\circ} \mathrm{C}$. This suggests that (i) product decomposition is an important factor at $110^{\circ} \mathrm{C}$ and (ii) that lower temperature favors site selectivity for $\mathrm{C}-\mathrm{H}$ amination of secondary $\mathrm{C}-\mathrm{H}$ bonds with lower bond dissociation energies than primary $\mathrm{C}-\mathrm{H}$ bonds.

\section{A. Primary vs. Secondary C-H Amination.}

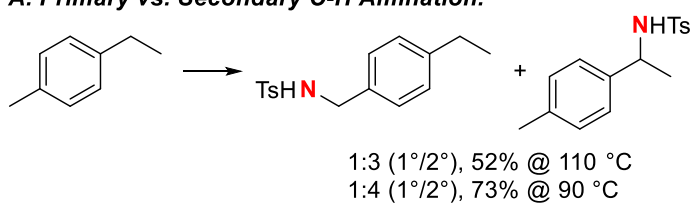

B. Secondary vs. Tertiary C-H Amination.

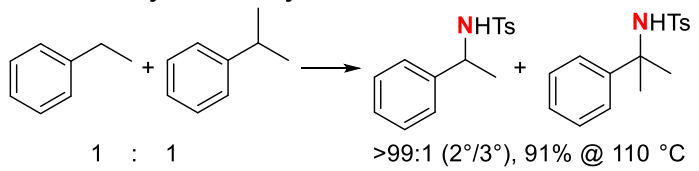

Scheme 8. Selectivity Studies. A. Primary vs. Secondary C-H Amination at 110 and $90{ }^{\circ} \mathrm{C}$. B. Secondary vs. Tertiary C-H Amination at $110^{\circ} \mathrm{C}$.

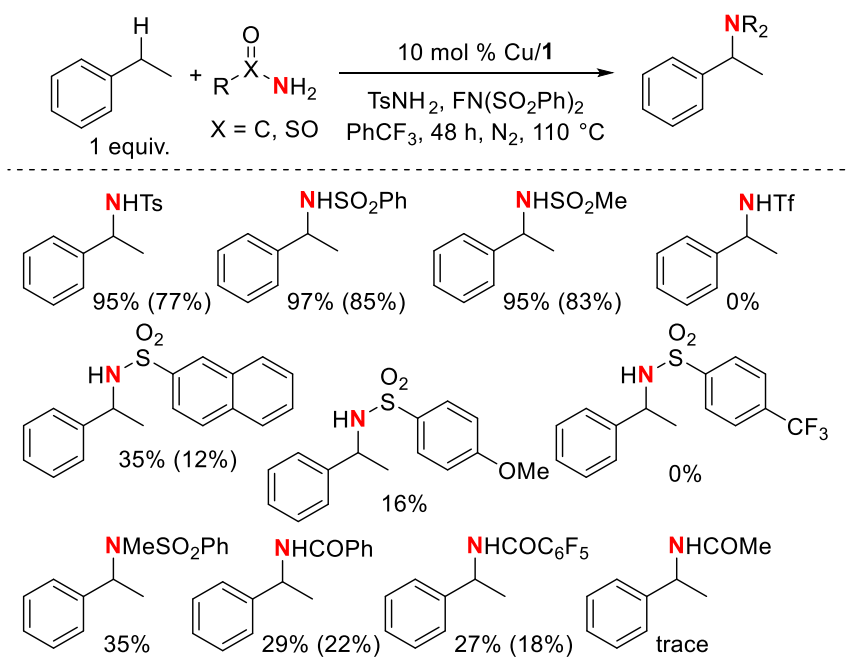

Scheme 9. Nitrogen Source Scope. Yields were determined by quantitative, crude ${ }^{1} \mathrm{H}$ NMR using $\mathrm{ClH}_{2} \mathrm{CCHCl}_{2}$ as internal standard. Yields are shown as averages of at least two catalytic runs. Isolated yields are shown in brackets.
In contrast to this effect on primary vs. secondary $\mathrm{C}-\mathrm{H}$ amination, amination is completely selective for transformation of secondary $\mathrm{C}-\mathrm{H}$ bonds vs. tertiary $\mathrm{C}-\mathrm{H}$ bonds (Scheme $8 \mathrm{~B}$ ). This implies that the reaction mechanism is unlikely to proceed through free radical intermediates, as at least small amounts of tertiary $\mathrm{C}-\mathrm{H}$ amination would be excepted in that case.

Next, we explored the scope of introducible $\mathrm{N}$ sources (Scheme 9). Generally, all reactions with electron-neutral $\left(\mathrm{H}_{2} \mathrm{NSO}_{2} \mathrm{R}, \mathrm{R}=p\right.$-tol, $\mathrm{Ph}$, naphthyl; 95\%, 97\%, 35\%) and electron-rich sulfonamides $\left(\mathrm{H}_{2} \mathrm{NSO}_{2} \mathrm{R}, \mathrm{R}=\mathrm{Me}, p-\mathrm{C}_{6} \mathrm{H}_{4} \mathrm{OMe} ; 95 \%\right.$, $16 \%$ ) formed the respective benzylic $\mathrm{C}-\mathrm{H}$ amination product, while electron-poor sulfonamides $\left(\mathrm{H}_{2} \mathrm{NSO}_{2} \mathrm{R}, \mathrm{R}=\mathrm{CF}_{3}\right.$, $p-\mathrm{C}_{6} \mathrm{H}_{4} \mathrm{CF}_{3}$ ) were unreactive. In contrast to previous work with this catalyst system, ${ }^{18}$ reactivity to the $\mathrm{C}-\mathrm{H}$ amination product was also observed with secondary sulfonamides $\left(\mathrm{HNMeSO}_{2} \mathrm{Ph}\right.$; $35 \%)$ and, most excitingly, benzamides as $\mathrm{N}$ source $\left(\mathrm{H}_{2} \mathrm{NCOPh}\right.$, $\left.29 \% ; \mathrm{H}_{2} \mathrm{NCOC}_{6} \mathrm{~F}_{5}, 27 \%\right)$. With acetamide, only traces $(<1 \%)$ of product were observed. Isolated yields generally aligned with the yields measured by quantitative ${ }^{1} \mathrm{H}$ NMR.

Several experimental studies were performed to gain insight into the reaction mechanism of the protocol. KIE studies with a $1: 1$ mixture of toluene and toluene- $\mathrm{d}_{8}$ as substrate resulted in an equimolar mixture of deuterated and non-deuterated $\mathrm{C}-\mathrm{H}$ amination product, implying a kinetic isotope effect (KIE) of 1.0. This suggests that $\mathrm{C}-\mathrm{H}$ bond scission in the developed reaction occurs after the rate-determining step and that $\mathrm{C}-\mathrm{H}$ bond cleavage is fast in comparison to other reaction steps. The obtained KIE value is further distinctly differentiated from KIEs observed in similar $\mathrm{Cu}$ catalyzed $\mathrm{C}-\mathrm{H}$ functionalizations, such as Stahl and Liu's C-H cyanation $(\mathrm{KIE}=3.5)$ via radical relay reactivity, ${ }^{19}$ Warren's $\mathrm{C}-\mathrm{H}$ amination employing a $\mathrm{Cu}$ (nacnac) catalyst system $(\mathrm{KIE}=70),{ }^{13}$ or Liu's Cu catalyzed $\mathrm{C}-\mathrm{H}$ amination with NFSI that is selective for primary benzylic $\mathrm{C}-\mathrm{H}$ amination $(\mathrm{KIE}=4.0){ }^{20}$

A series of radical trapping experiments with TEMPO, $\mathrm{BrCCl}_{3}$, and air did not provide further insight into the operating reaction mechanism (for details see the $\mathrm{SI}$ ). In all cases, substrate consumption seemed to be unaffected by the employed additives, while yields were lowered. As such, a radical relay as in Stahl and Liu's C-H cyanation ${ }^{19}$ seems unlikely, while other mechanisms could not be excluded based on the available data.

Overall, this manuscript summarizes the development of a reaction protocol that addresses two common drawbacks in nondirected, intermolecular benzylic $\mathrm{C}-\mathrm{H}$ aminations: (i) the need to use an excess of substrate and (ii) the limitation to only introduce one type of nitrogen source. The strong oxidant $\mathrm{N}$-fluorobenzenesulfonimide (NFSI) in combination with a $\mathrm{Cu} /$ diimine ligand catalyst system are important to achieve this reactivity and allow low substrate loadings of 1.0 equivalent. Under these conditions, up to $97 \%$ yield of $\mathrm{C}-\mathrm{H}$ amination product can be obtained. Excitingly, the use of both sulfonamides and benzamides as nitrogen sources is tolerated. The ability to introduce various protected amine groups suggests (in agreement with findings by Warren and co-workers $)^{12-15}$ that combinations of nitrogen sources and oxidants in $\mathrm{Cu}$ catalyzed benzylic $\mathrm{C}-\mathrm{H}$ aminations are synthetically more versatile with respect to their product scope than approaches employing electrophilic amination reagents, such as Chloramine-T, ${ }^{21-23}$ protected hydroxylamines, ${ }^{18}$ or reagents derived from iodosoarenes (e.g. PhI=NTs). 


\section{ASSOCIATED CONTENT}

Experimental procedures, spectroscopic data, and detailed optimization data (PDF) are attached to this manuscript.

\section{AUTHOR INFORMATION}

\section{Corresponding Author}

*mhemmert@wpi.edu; marion.emmert@merck.com; @EmmertLab

\section{Author Contributions}

The manuscript was written through contributions of all authors. All authors have given approval to the final version of the manuscript.

\section{ACKNOWLEDGMENT}

We acknowledge funding of this work by the NIH (National Institute of General Medical Sciences award 1R15GM107939-01A1).

\section{REFERENCES}

(1) C-H Bond Activation in Organic Synthesis, Li, J. J., Ed. CRC Press: 2015.

(2) Davies, H. M. L.; Du Bois, J.; Yu, J.-Q., C-H Functionalization in Organic Synthesis. Chem. Soc. Rec. 2011, 40, 1855-1856.

(3) Gutekunst, W. R.; Baran, P. S., C-H Functionalization Logic in Total Synthesis. Chem. Soc. Rev. 2011, 40, 1976-1991.

(4) Wencel-Delord, J.; Droge, T.; Liu, F.; Glorius, F., Towards Mild Metal-Catalyzed C-H Bond Activation. Chem. Soc. Rev. 2011, 40, 4740-4761.

(5) Giri, R.; Shi, B.-F.; Engle, K. M.; Maugel, N.; Yu, J.-Q., Transition Metal-Catalyzed C-H Activation Reactions: Diastereoselectivity and Enantioselectivity. Chem. Soc. Rev. 2009, 38, 3242-3272.

(6) Shilov, A. E.; Shul'pin, G. B., Activation of C-H Bonds by Metal Complexes. Chem. Rev. 1997, 97, 2879-2932.

(7) Emmert, M. H.; Legacy, C. J., Chelate-Assisted Arene C-H Bond Functionalization. In Arene chemistry: reaction mechanisms and methods for aromatic compounds, Mortier, J., Ed. Wiley: 2016; pp 647-674.

(8) Lyons, T. W.; Sanford, M. S., Palladium-Catalyzed Ligand-Directed C-H Functionalization Reactions. Chem. Rev. 2010, 110, 1147 1169.

(9) Ritleng, V.; Sirlin, C.; Pfeffer, M., Ru-, Rh-, and Pd-Catalyzed C-C Bond Formation Involving C-H Activation and Addition on Unsaturated Substrates: Reactions and Mechanistic Aspects. Chem. Rev. 2002, 102, 1731-1770.
(10) Kuhl, N.; Hopkinson, M. N.; Wencel-Delord, J.; Glorius, F., Beyond Directing Groups: Transition-Metal-Catalyzed C-H Activation of Simple Arenes. Angew. Chem. Int. Ed. 2012, 51, 10236-10254.

(11) Kalyani, D.; Marlier, E. E., Reactivity and Selectivity in Transition Metal-Catalyzed, Nondirected Arene Functionalizations. In Arene chemistry: reaction mechanisms and methods for aromatic compounds, Mortier, J., Ed. Wiley: 2016; pp 675-714.

(12) Wiese, S.; Badiei, Y. M.; Gephart, R. T.; Mossin, S.; Varonka, M. S.; Melzer, M. M.; Meyer, K.; Cundari, T. R.; Warren, T. H., Catalytic C-H Amination with Unactivated Amines through Copper(II) Amides. Angew. Chem. Int. Ed. 2010, 49, 8850-8855.

(13) Gephart, R. T.; Huang, D. L.; Aguila, M. J.; Schmidt, G.; Shahu, A.; Warren, T. H., Catalytic C-H Amination with Aromatic Amines. Angew. Chem. Int. Ed. 2012, 51, 6488-6492.

(14) Gephart, R. T.; Warren, T. H., Copper-Catalyzed sp ${ }^{3}$ C-H Amination. Organometallics 2012, 31, 7728-7752.

(15) Jang, E. S.; McMullin, C. L.; Kass, M.; Meyer, K.; Cundari, T. R.; Warren, T. H., Copper(II) Anilides in $\mathrm{sp}^{3} \mathrm{C}-\mathrm{H}$ Amination. J. Am. Chem. Soc. 2014, 136, 10930-10940.

(16) Zeng, H. T.; Huang, J. M., Copper-Catalyzed Ligand-Free Amidation of Benzylic Hydrocarbons and Inactive Aliphatic Alkanes. Org. Lett. 2015, 17, 4276-4279.

(17) Diaz-Requejo, M. M.; Perez, P. J., Coinage Metal Catalyzed CH Bond Functionalization of Hydrocarbons. Chem. Rev. 2008, 108, 3379-3394.

(18) Wang, A.; Venditto, N. J.; Darcy, J. W.; Emmert, M. H., Nondirected, $\mathrm{Cu}$-Catalyzed $\mathrm{sp}^{3} \mathrm{C}-\mathrm{H}$ Amination with Hydroxylamine-Based Amination Reagents: Catalytic and Mechanistic Studies. Organometallics 2017, 36, 1259-1268.

(19) Zhang, W.; Wang, F.; McCann, S. D.; Wang, D.; Chen, P.; Stahl, S. S.; Liu, G., Enantioselective Cyanation of Benzylic C-H Bonds via Copper-Catalyzed Radical Relay. Science 2016, 353, 10141018.

(20) Ni, Z.; Zhang, Q.; Xiong, T.; Zheng, Y.; Li, Y.; Zhang, H.; Zhang, J.; Liu, Q., Highly Regioselective Copper-Catalyzed Benzylic $\mathrm{C}-\mathrm{H}$ Amination by $\mathrm{N}$-fluorobenzenesulfonimide. Angew. Chem. Int Ed. 2012, 51, 1244-1247.

(21) Barman, D. N.; Nicholas, K. M., Ligand-Assisted, Copper-Catalyzed Enantioselective Benzylic Amination. Tetrahedron Lett. 2010, $51,1815-1818$

(22) Barman, D. N.; Liu, P.; Houk, K. N.; Nicholas, K. M., On the Mechanism of Ligand-Assisted, Copper-Catalyzed Benzylic Amination by Chloramine-T. Organometallics 2010, 29, 3404-3412.

(23) Bhuyan, R.; Nicholas, K. M., Efficient Copper-Catalyzed Benzylic Amidation with Anhydrous Chloramine-T. Org. Lett. 2007, 9, 3957-3959. 


\title{
Non-Directed, Copper Catalyzed Benzylic C-H Amination Avoiding Substrate Excess
}

\author{
Anqi Wang, ${ }^{\dagger}$ Cristina C. DeOliveira, ${ }^{\dagger}$ and Marion H. Emmert ${ }^{\dagger+*}$ \\ †Department of Chemistry and Biochemistry, Worcester Polytechnic Institute, 100 Institute Road, Worcester, MA \\ 01609, U.S.A. \\ †Current Address: Process Research \& Development, MRL, Merck \& Co. Inc, 770 Sumneytown Pike, West Point, \\ PA, 19486, U.S.A.
}




\section{Table of Contents}

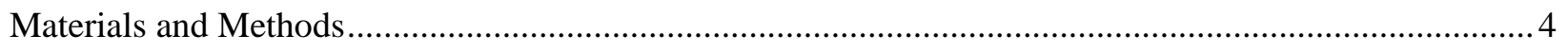

Representative Procedure for Catalytic Benzylic Amination Reactions ............................................... 5

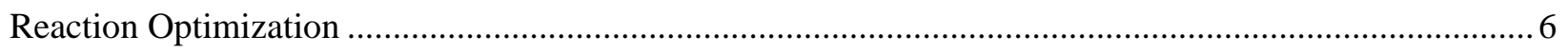

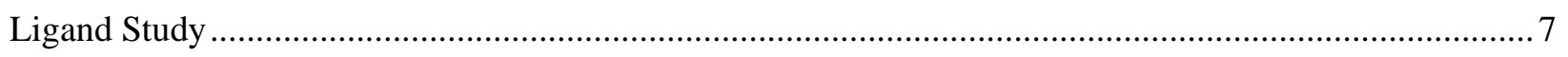

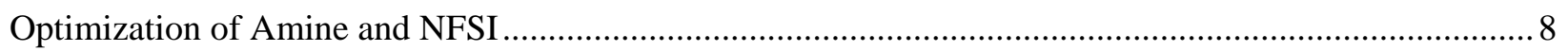

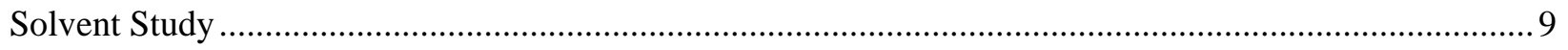

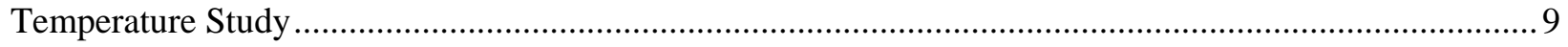

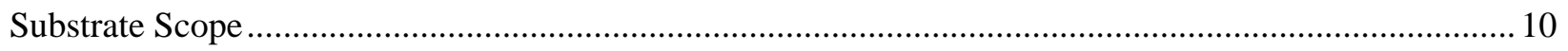

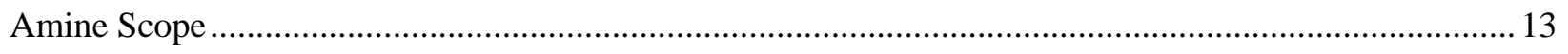

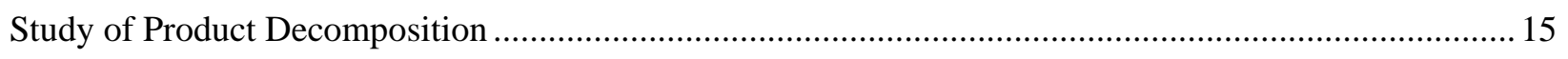

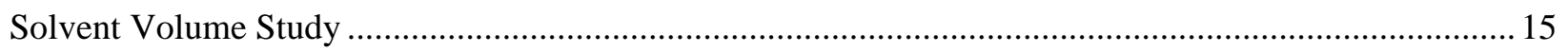

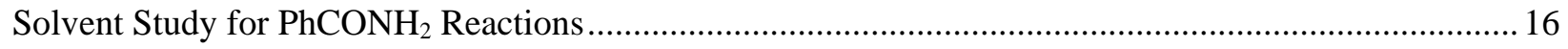

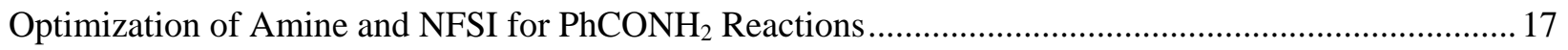

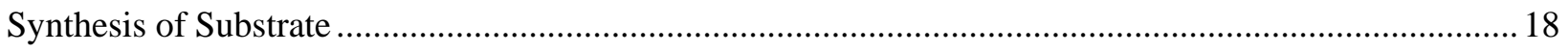

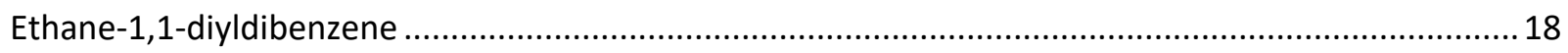

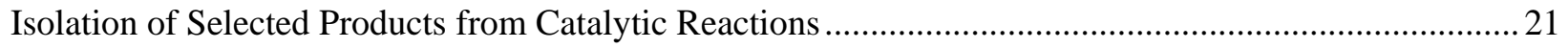

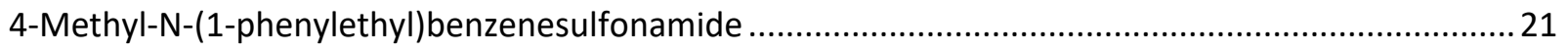

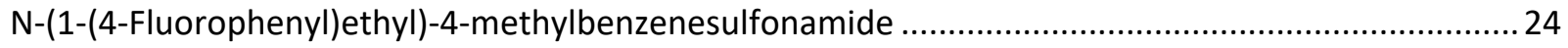

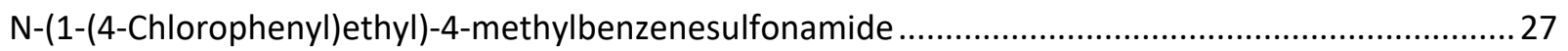

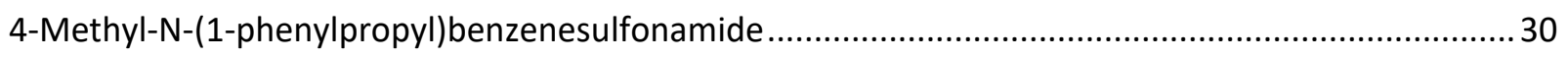

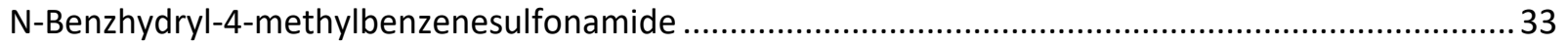

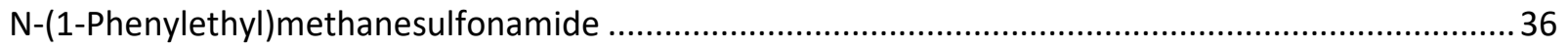

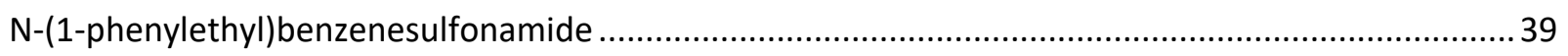

4-Methyl-N-(1-(naphthalen-2-yl)ethyl)benzenesulfonamide ......................................................... 42

N,4-Dimethyl-N-(1-phenylethyl)benzenesulfonamide .................................................................. 45

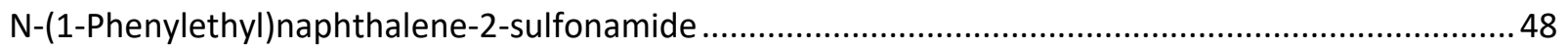

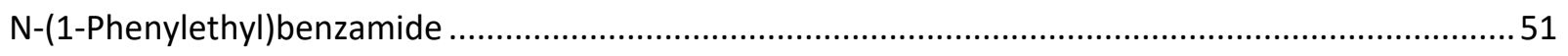

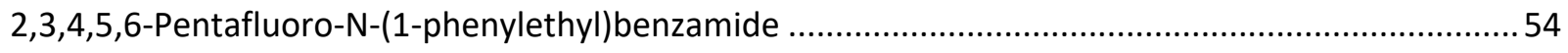

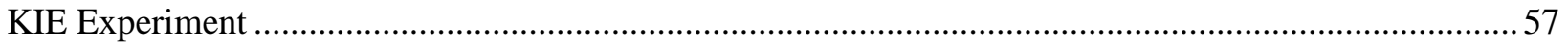

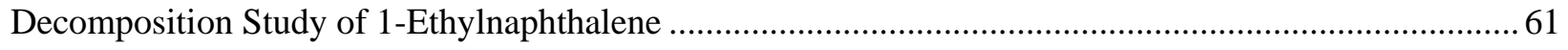




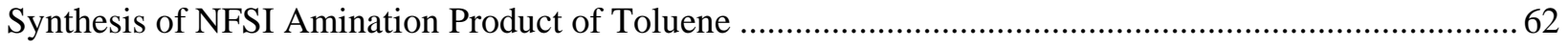

Catalytic reaction with NFSI Amination Product of Toluene $-\mathrm{PhCH}_{2}\left(\mathrm{NSO}_{2} \mathrm{Ph}\right)_{2} \ldots \ldots \ldots \ldots \ldots \ldots \ldots \ldots \ldots \ldots . . . . . . . . . . . . . . . . . .65$ Side Product Analysis .................................................................................. Error! Bookmark not defined.

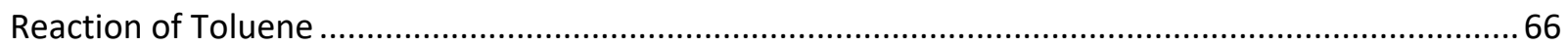

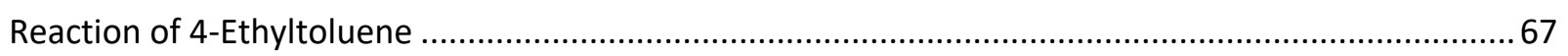

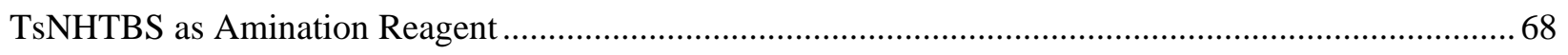

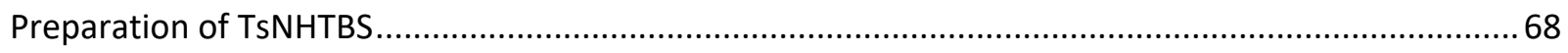

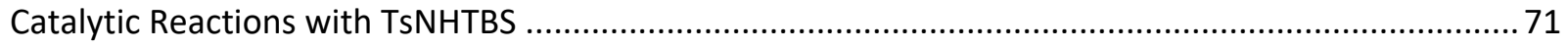

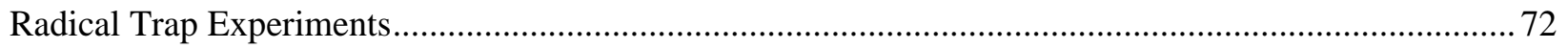

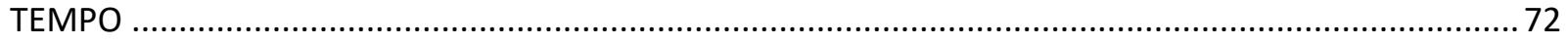

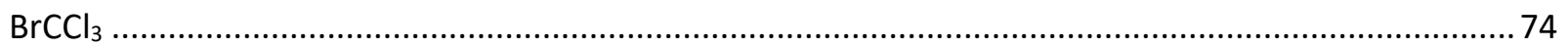

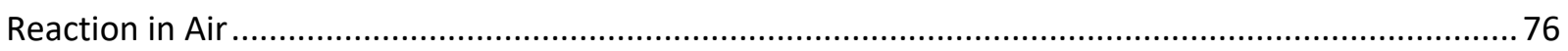

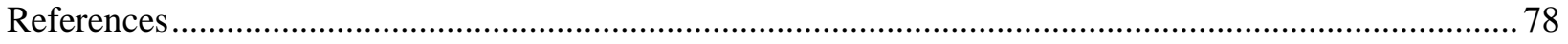




\section{Materials and Methods}

All reagents and solvents were used as received unless noted otherwise. All reagents were obtained commercially unless otherwise noted.

Stirbars used in catalytic reactions were cleaned with aqua regia for at least $3 \mathrm{~h}$ upon gentle stirring, rinsed with copious amounts of water, and dried in an oven at $120{ }^{\circ} \mathrm{C}$ prior to use. Yields are reported as average yields of at least 2 experiments. The reported error is the standard deviation of at least two replicate trials.

All catalytic experiments were set up in a Vigor glovebox kept under a dry nitrogen atmosphere unless otherwise noted. Upon sealing the reaction vials in the glovebox, catalytic reaction mixtures were removed from the glovebox and heated on pre-heated vial plates.

All NMR experiments were carried out on a Bruker BioSpin 500MHz Avance III Digital NMR spectrometer. All quantitative ${ }^{1} \mathrm{H}$ NMR measurements were performed using an adjusted method (15 s relaxation time, NS = 32) with 1,1,2-trichloroethane as internal standard. All NMR spectra were recorded at room temperature unless otherwise noted.

GC-MS analyses were performed on an Agilent 5975C instrument using a 19091S-433 (HP-5MS; $30 \mathrm{~m}, 0.25 \mathrm{~mm}$ i.d., $0.25 \mu \mathrm{m} \mathrm{df}$ ) column. The identities of all products were verified by comparison of the obtained data with NMR and GC-MS data of original samples. 


\section{Representative Procedure for Catalytic Benzylic Amination Reactions}

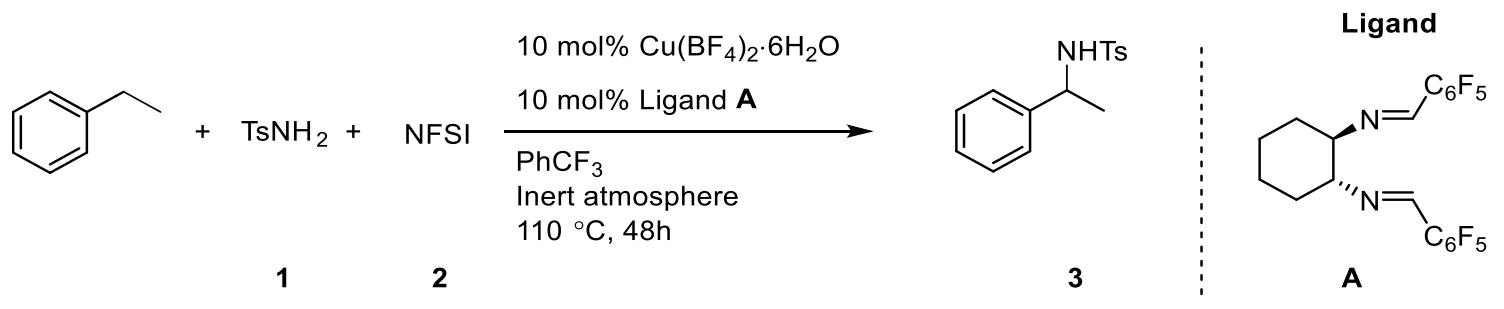

In a glovebox, $\mathrm{Cu}\left(\mathrm{BF}_{4}\right)_{2} \cdot 6 \mathrm{H}_{2} \mathrm{O}(4.6 \mathrm{mg}, 12.6 \mu \mathrm{mol}, 10.0 \mathrm{~mol} \%)$, ligand $\mathbf{A}(6.0 \mathrm{mg}, 12.6$ $\mu \mathrm{mol}, \quad 10.0 \mathrm{~mol} \%)$, p-Toluenesulfonamide $(64.2 \mathrm{mg}, 0.375 \mathrm{mmol}, 3.00$ equiv.), $N-$ Fluorobenzenesulfonimide ( $118.3 \mathrm{mg}, 0.375 \mathrm{mmol}, 3.00$ equiv.), ethylbenezene (16.0 $\mu \mathrm{L}, 13.3$ $\mathrm{mg}, 0.125 \mathrm{mmol}, 1.00$ equiv.) and $1.5 \mathrm{~mL}$ benzotrifluoride were added in this sequence to a $4 \mathrm{~mL}$ scintillation vial, equipped with a Teflon-coated stir bar. The vial was sealed with a Teflonlined vial cap and heated to $110{ }^{\circ} \mathrm{C}$ on a pre-heated vial plate under vigorous stirring (1500 rpm). After stirring the mixture vigorously for the dedicated reaction time, the vial was taken off the heating block and the mixture was allowed to cool to room temperature. The solvent was removed under reduced pressure (rotary evaporator). 1,1,2-trichloroethane $(20.0 \mu \mathrm{L}, 28.8 \mathrm{mg}$, $215.9 \mathrm{mmol}$ ) was added into the reaction vial as NMR standard following the addidtion of 1.0 $\mathrm{mL} \mathrm{CDCl}$. The resulting mixture was filtered through Celite. The filtrate was used directly for quantitative ${ }^{1} \mathrm{H}$ NMR measurements (as shown in Figure S1) to determine the yield of product 3 by measuring the ratio of integrals for the benzylic proton of $\mathbf{3}$ (peak at $4.53 \mathrm{ppm}$ ) and the proton of 1,1,2-trichloroethane at $5.79 \mathrm{ppm}$.

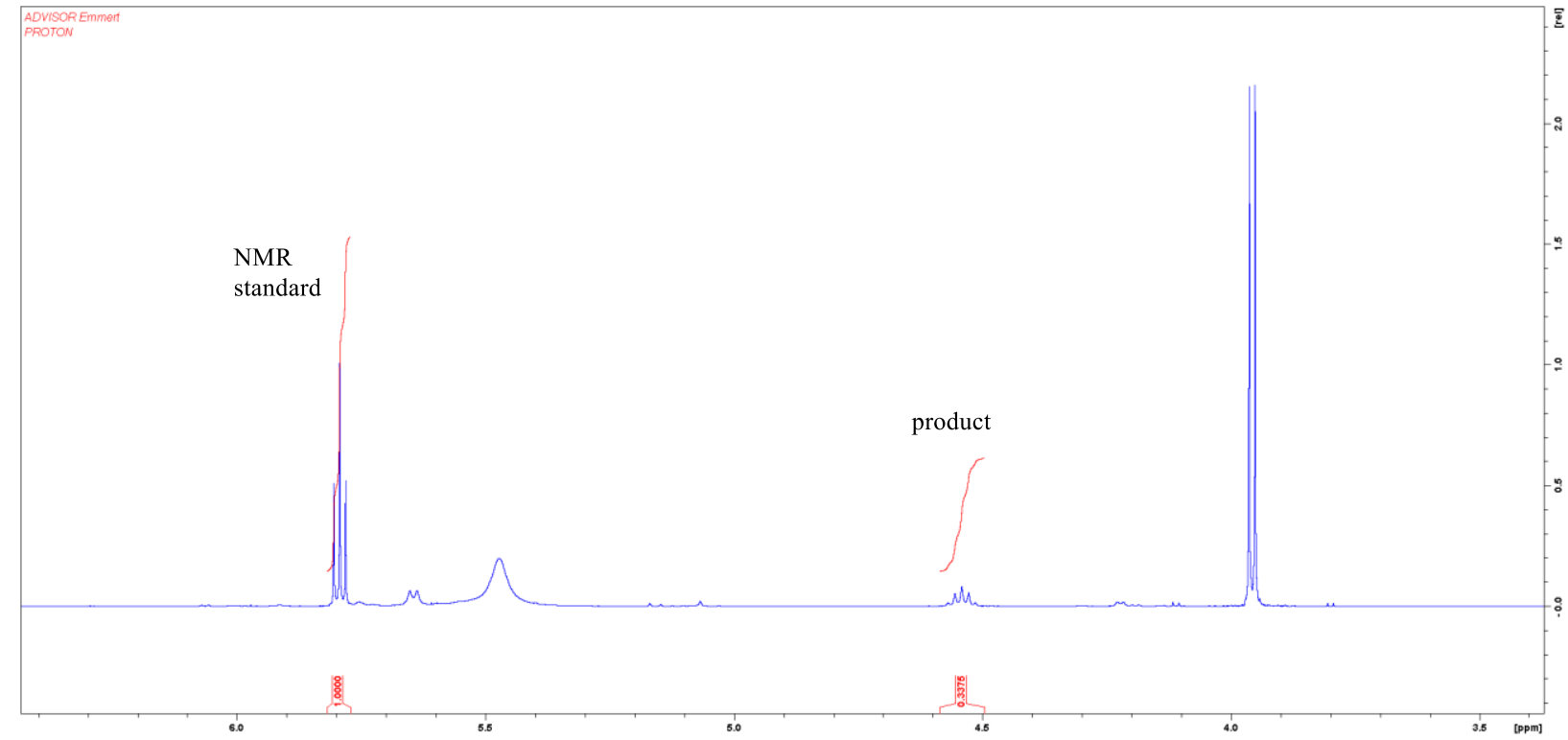

Figure S1. NMR Example for Yield Analysis of Product 


\section{Reaction Optimization}

Table S1. Reaction Optimization. Conditions: Ethylbenzene (16.0 $\mu \mathrm{L}, 13.3 \mathrm{mg}, 0.125 \mathrm{mmol}, 1.00$ equiv.), amination reagent 1 (p-Toluenesulfonamide, $64.2 \mathrm{mg}, 0.375 \mathrm{mmol}, 3.00$ equiv.), $N$-Fluorobenzenesulfonimide (118.3 mg, $0.375 \mathrm{mmol}, 3.00$ equiv.) and $1.5 \mathrm{~mL}$ benzotrifluoride $110{ }^{\circ} \mathrm{C}, 48 \mathrm{~h}$. The reactions were prepared according to the Representative Procedure for Catalytic Benzylic Amination Reactions.

\begin{tabular}{|c|c|c|c|c|c|}
\hline \multicolumn{4}{|c|}{$\begin{array}{l}\text { Cu Precatalyst } \\
\text { Ligand } \\
\mathrm{PhCF}_{3} \\
110^{\circ} \mathrm{C}, 48 \mathrm{~h}\end{array}$} & \multicolumn{2}{|c|}{$\underset{\mid}{\mathrm{NHT}}$} \\
\hline Entry & Copper precatalyst & Ligand & NFSI & Atmosphere & NMR Yield of $\mathbf{3}$ \\
\hline 1 & - & - & Yes & air & $0 \pm 0$ \\
\hline 2 & - & - & 3.00 equiv & nitrogen & $0 \pm 0$ \\
\hline 3 & $\mathrm{Cu}\left(\mathrm{BF}_{4}\right)_{2} \cdot 6 \mathrm{H}_{2} \mathrm{O}(10 \mathrm{~mol} \%)$ & - & 3.00 equiv & air & $51 \pm 2$ \\
\hline 4 & $\mathrm{Cu}\left(\mathrm{BF}_{4}\right)_{2} \cdot 6 \mathrm{H}_{2} \mathrm{O}(10 \mathrm{~mol} \%)$ & $\mathbf{A}(10 \mathrm{~mol} \%)$ & 3.00 equiv & air & $64 \pm 2$ \\
\hline 5 & - & - & No NFSI & nitrogen & $0 \pm 0$ \\
\hline 6 & $\mathrm{Cu}\left(\mathrm{BF}_{4}\right)_{2} \cdot 6 \mathrm{H}_{2} \mathrm{O}(10 \mathrm{~mol} \%)$ & - & 3.00 equiv & nitrogen & $75 \pm 1$ \\
\hline 7 & $\mathrm{CuCl}(10 \mathrm{~mol} \%)$ & - & 3.00 equiv & nitrogen & $65 \pm 2$ \\
\hline 8 & $\mathrm{CuBr}(10 \mathrm{~mol} \%)$ & - & 3.00 equiv & nitrogen & $0 \pm 0$ \\
\hline 9 & $\mathrm{CuCl}_{2}(10 \mathrm{~mol} \%)$ & - & 3.00 equiv & nitrogen & $68 \pm 1$ \\
\hline 10 & {$\left[\mathrm{Cu}(\mathrm{MeCN})_{4}\right] \mathrm{PF}_{6}(10 \mathrm{~mol} \%)$} & - & 3.00 equiv & nitrogen & $73 \pm 1$ \\
\hline 11 & $\mathrm{Cu}(\mathrm{OAc})_{2}(10 \mathrm{~mol} \%)$ & - & 3.00 equiv & nitrogen & $63 \pm 2$ \\
\hline 12 & $\mathrm{Cu}(\mathrm{OTf})_{2}(10 \mathrm{~mol} \%)$ & - & 3.00 equiv & nitrogen & $7 \pm 0$ \\
\hline 13 & CuOTf $\cdot 0.5$ toluene $(10 \mathrm{~mol} \%)$ & - & 3.00 equiv & nitrogen & $41 \pm 1$ \\
\hline 14 & $\mathrm{Cu}\left(\mathrm{BF}_{4}\right)_{2} \cdot 6 \mathrm{H}_{2} \mathrm{O}(10 \mathrm{~mol} \%)$ & $\mathbf{A}(10 \mathrm{~mol} \%)$ & 3.00 equiv & nitrogen & $95 \pm 2$ \\
\hline 15 & $\mathrm{Cu}\left(\mathrm{BF}_{4}\right)_{2} \cdot 6 \mathrm{H}_{2} \mathrm{O}(10 \mathrm{~mol} \%)$ & B (10 mol\%) & 3.00 equiv & nitrogen & $32 \pm 2$ \\
\hline 16 & $\mathrm{Cu}\left(\mathrm{BF}_{4}\right)_{2} \cdot 6 \mathrm{H}_{2} \mathrm{O}(10 \mathrm{~mol} \%)$ & $\mathbf{C}(10 \mathrm{~mol} \%)$ & 3.00 equiv & nitrogen & $76 \pm 2$ \\
\hline 17 & $\mathrm{Cu}\left(\mathrm{BF}_{4}\right)_{2} \cdot 6 \mathrm{H}_{2} \mathrm{O}(10 \mathrm{~mol} \%)$ & D (10 mol\%) & 3.00 equiv & nitrogen & $72 \pm 2$ \\
\hline 18 & $\mathrm{Cu}\left(\mathrm{BF}_{4}\right)_{2} \cdot 6 \mathrm{H}_{2} \mathrm{O}(10 \mathrm{~mol} \%)$ & $\mathbf{E}(10 \mathrm{~mol} \%)$ & 3.00 equiv & nitrogen & $57 \pm 1$ \\
\hline 19 & $\mathrm{Cu}\left(\mathrm{BF}_{4}\right)_{2} \cdot 6 \mathrm{H}_{2} \mathrm{O}(10 \mathrm{~mol} \%)$ & $\mathbf{F}(10 \mathrm{~mol} \%)$ & 3.00 equiv & nitrogen & $72 \pm 1$ \\
\hline
\end{tabular}<smiles>Fc1c(F)c(F)c(C=N[C@@H]2CCCC[C@@H]2/N=C/c2c(F)c(F)c(F)c(F)c2F)c(F)c1F</smiles>

A<smiles>Clc1cccc(Cl)c1C=N[C@H]1CCCC[C@H]1N=Cc1c(Cl)cccc1Cl</smiles>

D

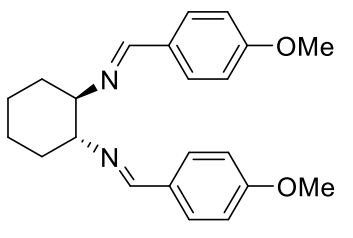

B

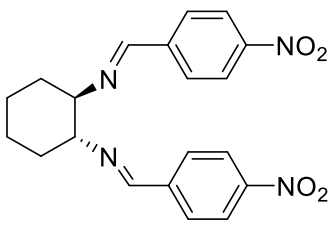

$\mathbf{E}$<smiles>Fc1cccc(F)c1C=N[C@H]1CCCC[C@H]1N=Cc1c(F)cccc1F</smiles>

C

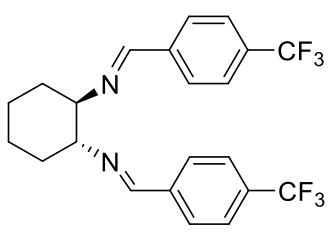




\section{Ligand Study}

Table S2. Ligand Study@ $90{ }^{\circ} \mathbf{C}$ and $110{ }^{\circ} \mathbf{C}$. Ethylbenzene $(16.0 \mu \mathrm{L}, 13.3 \mathrm{mg}, 0.125 \mathrm{mmol}, 1.00$ equiv.), $\mathrm{Cu}\left(\mathrm{BF}_{4}\right)_{2} \cdot 6 \mathrm{H}_{2} \mathrm{O}(4.6 \mathrm{mg}, 12.6 \mu \mathrm{mol}, 10.0 \mathrm{~mol} \%)$, ligand $(12.6 \mu \mathrm{mol}, 10.0 \mathrm{~mol} \%)$, amination reagent 1 (pToluenesulfonamide, $64.2 \mathrm{mg}, 0.375 \mathrm{mmol}, 3.00$ equiv.), $N$-Fluorobenzenesulfonimide ( $118.3 \mathrm{mg}, 0.375 \mathrm{mmol}, 3.00$ equiv.) and $1.5 \mathrm{~mL}$ benzotrifluoride $110{ }^{\circ} \mathrm{C}, 48 \mathrm{~h}$. The reactions were prepared according to the Representative Procedure for Catalytic Benzylic Amination Reactions.

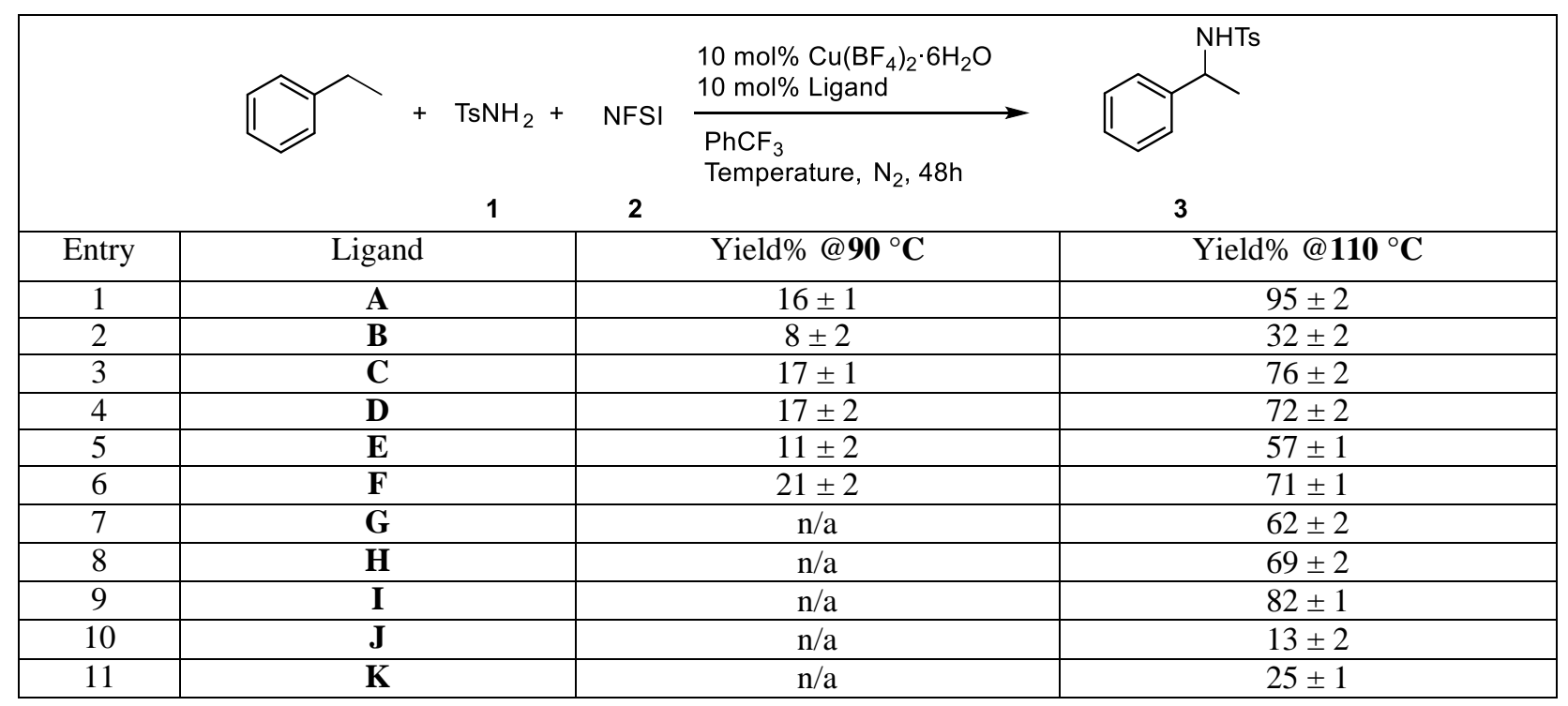



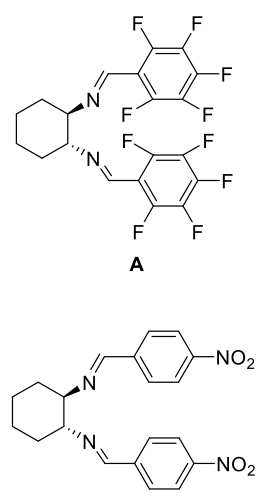

E

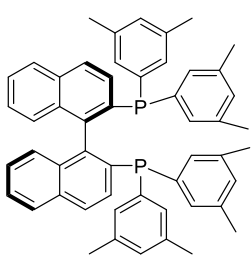

(R)-DM-BINAP
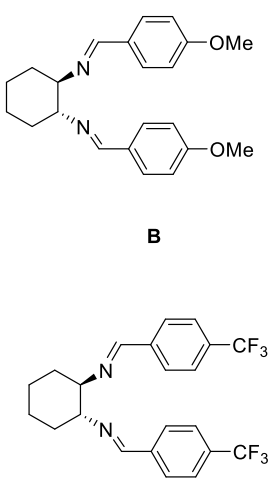

$\mathbf{F}$
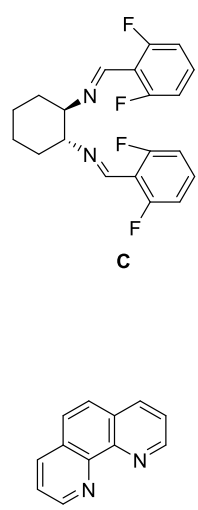

G<smiles>Pc1ccccc1-c1ccc2c(c1-c1c(-c3ccccc3)ccc3c1CCCC3)CCCC2</smiles>

(R)-H8-BINAP<smiles>Clc1ccccc1Cl</smiles>

D

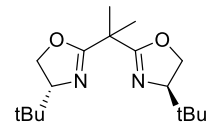

H

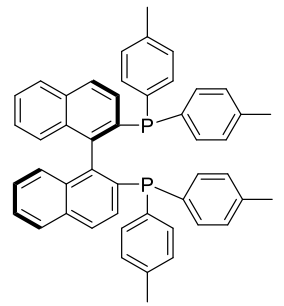

(R)-(-)-ToIBINAP

I

K

\section{Optimization of Amine and NFSI}

Table S3. Optimization of Amine and NFSI. Ethylbenzene ( $16.0 \mu \mathrm{L}, 13.3 \mathrm{mg}, 0.125 \mathrm{mmol}, 1.00$ equiv.), $\mathrm{Cu}\left(\mathrm{BF}_{4}\right)_{2} \cdot 6 \mathrm{H}_{2} \mathrm{O}(4.6 \mathrm{mg}, 12.6 \mu \mathrm{mol}, 10.0 \mathrm{~mol} \%)$, ligand $(12.6 \mu \mathrm{mol}, 10.0 \mathrm{~mol} \%)$, amination reagent $\mathbf{1}, \mathrm{N}$ -

Fluorobenzenesulfonimide and $1.5 \mathrm{~mL}$ benzotrifluoride $110^{\circ} \mathrm{C}, 48 \mathrm{~h}$. The reactions were prepared according to the Representative Procedure for Catalytic Benzylic Amination Reactions.

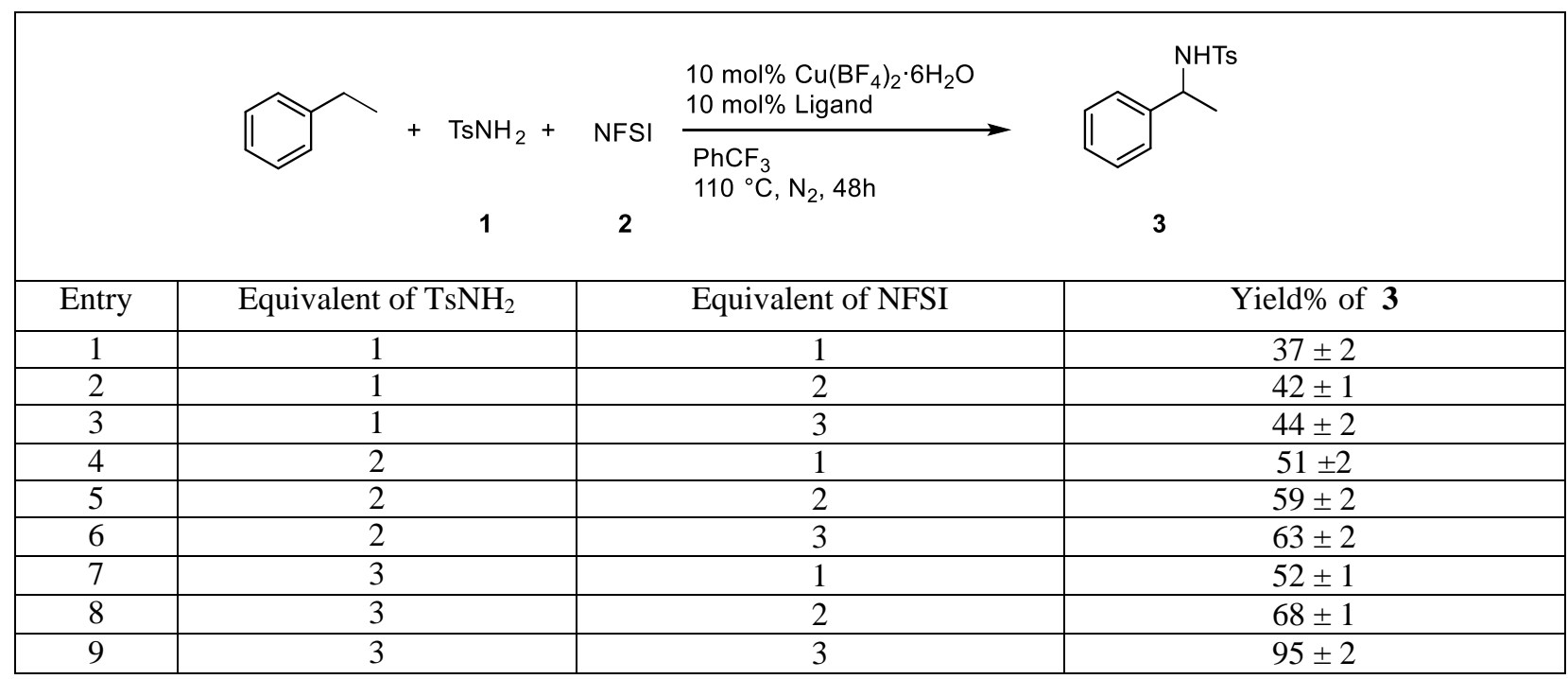




\section{Solvent Study}

Table S4. Solvent Study. Ethylbenzene ( $16.0 \mu \mathrm{L}, 13.3 \mathrm{mg}, 0.125 \mathrm{mmol}, 1.00$ equiv.), $\mathrm{Cu}\left(\mathrm{BF}_{4}\right)_{2} \cdot 6 \mathrm{H}_{2} \mathrm{O}(4.6 \mathrm{mg}, 12.6$ $\mu \mathrm{mol}, 10.0 \mathrm{~mol} \%)$, ligand A $(6.0 \mathrm{mg}, 12.6 \mu \mathrm{mol}, 10.0 \mathrm{~mol} \%)$, amination reagent $\mathbf{1}$ (p-Toluenesulfonamide, $64.2 \mathrm{mg}$, $0.375 \mathrm{mmol}, 3.00$ equiv.), $N$-Fluorobenzenesulfonimide ( $118.3 \mathrm{mg}, 0.375 \mathrm{mmol}, 3.00$ equiv.) and $1.5 \mathrm{~mL}$ solvent, $110{ }^{\circ} \mathrm{C}, 48 \mathrm{~h}$. The reactions were prepared according to the Representative Procedure for Catalytic Benzylic Amination Reactions.

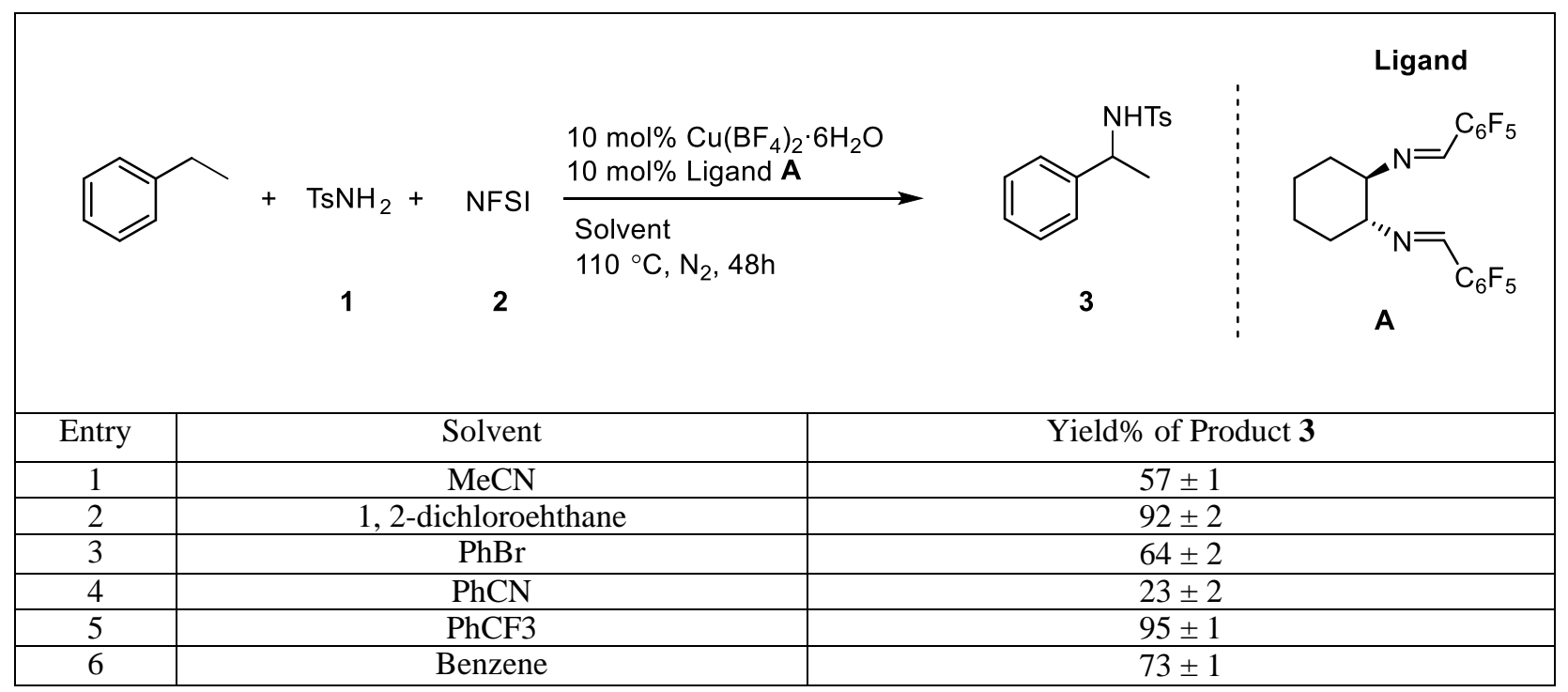

\section{Temperature Study}

Table S5. Temperature Study. Ethylbenzene $\left(16.0 \mu \mathrm{L}, 13.3 \mathrm{mg}, 0.125 \mathrm{mmol}, 1.00\right.$ equiv.), $\mathrm{Cu}\left(\mathrm{BF}_{4}\right)_{2} \cdot 6 \mathrm{H}_{2} \mathrm{O}(4.6$ $\mathrm{mg}, 12.6 \mu \mathrm{mol}, 10.0 \mathrm{~mol} \%)$, ligand $\mathbf{A}(6.0 \mathrm{mg}, 12.6 \mu \mathrm{mol}, 10.0 \mathrm{~mol} \%)$, amination reagent $\mathbf{1}$ (p-Toluenesulfonamide, $64.2 \mathrm{mg}, 0.375 \mathrm{mmol}, 3.00$ equiv.), $N$-Fluorobenzenesulfonimide (118.3 mg, $0.375 \mathrm{mmol}, 3.00$ equiv.) and $1.5 \mathrm{~mL}$ solvent, $48 \mathrm{~h}$. The reactions were prepared according to the Representative Procedure for Catalytic Benzylic Amination Reactions.

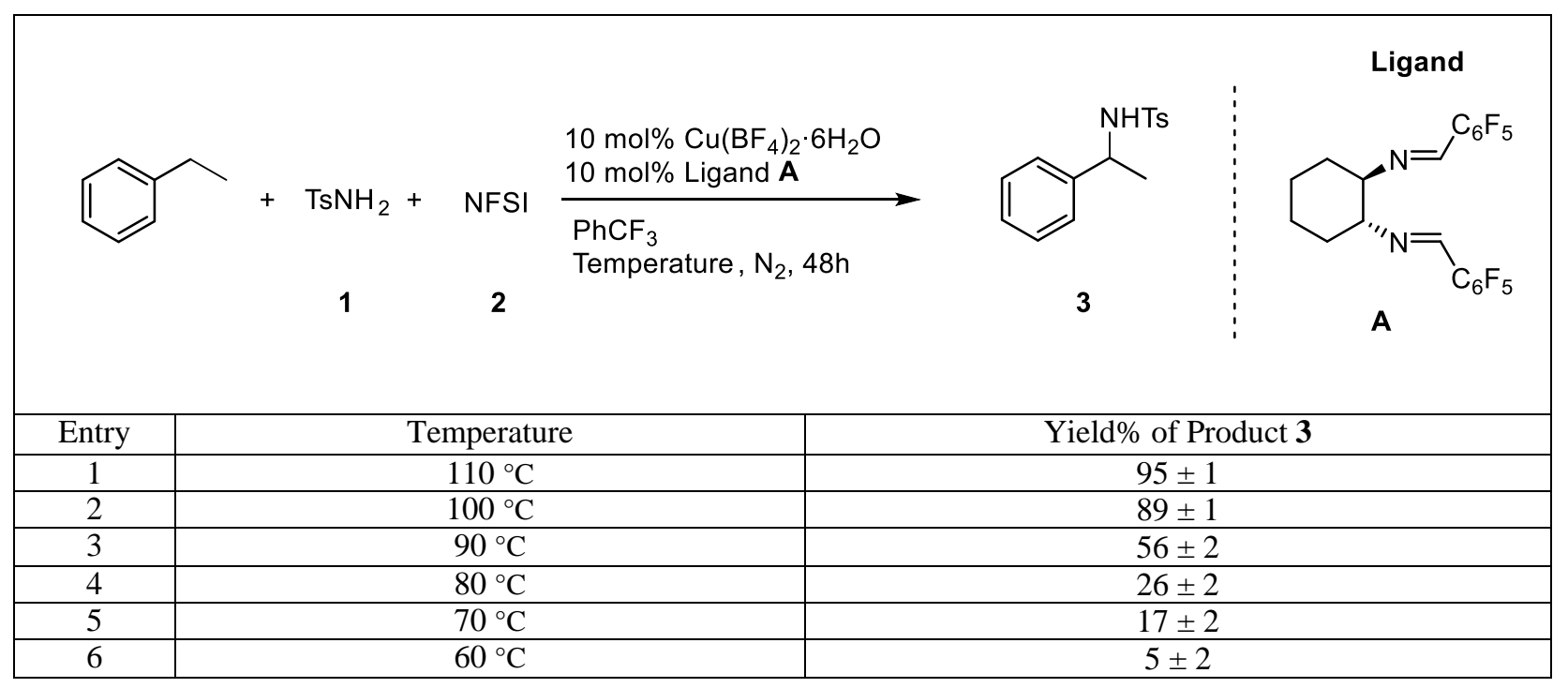




\section{Substrate Scope}

Table S6. Substrate Scope. Substrate $\left(0.125 \mathrm{mmol}, 1.00\right.$ equiv.), $\mathrm{Cu}\left(\mathrm{BF}_{4}\right)_{2} \cdot 6 \mathrm{H}_{2} \mathrm{O}(4.6 \mathrm{mg}, 12.6 \mu \mathrm{mol}, 10.0 \mathrm{~mol} \%)$, ligand $\mathbf{A}(6.0 \mathrm{mg}, 12.6 \mu \mathrm{mol}, 10.0 \mathrm{~mol} \%)$, amination reagent $\mathbf{1}$ (p-Toluenesulfonamide, $64.2 \mathrm{mg}, 0.375 \mathrm{mmol}, 3.00$ equiv.), $N$-Fluorobenzenesulfonimide (118.3 mg, $0.375 \mathrm{mmol}, 3.00$ equiv.) and $1.5 \mathrm{~mL} \mathrm{PhCF}_{3}, 48 \mathrm{~h}$. The reactions were prepared according to the Representative Procedure for Catalytic Benzylic Amination Reactions.

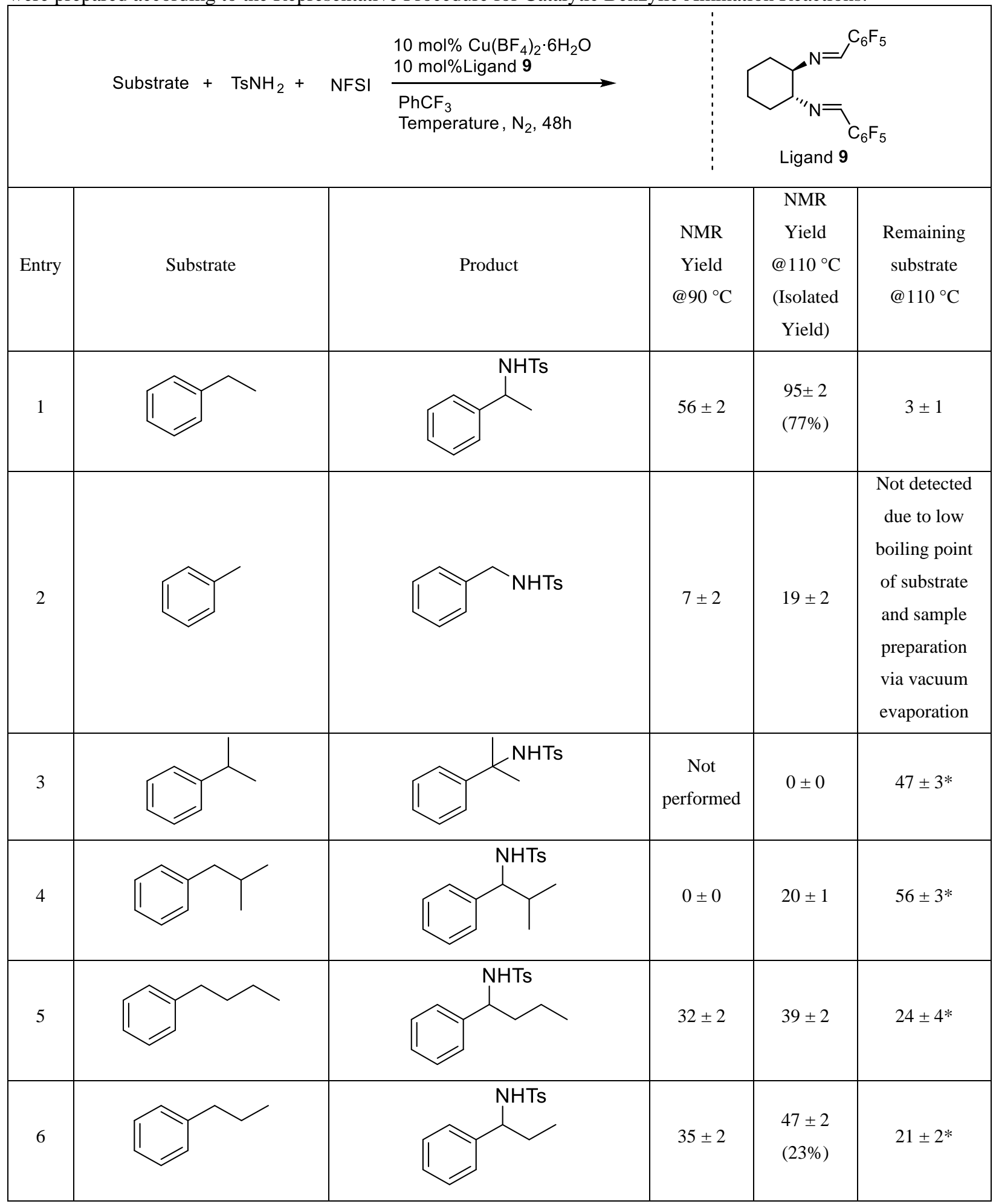




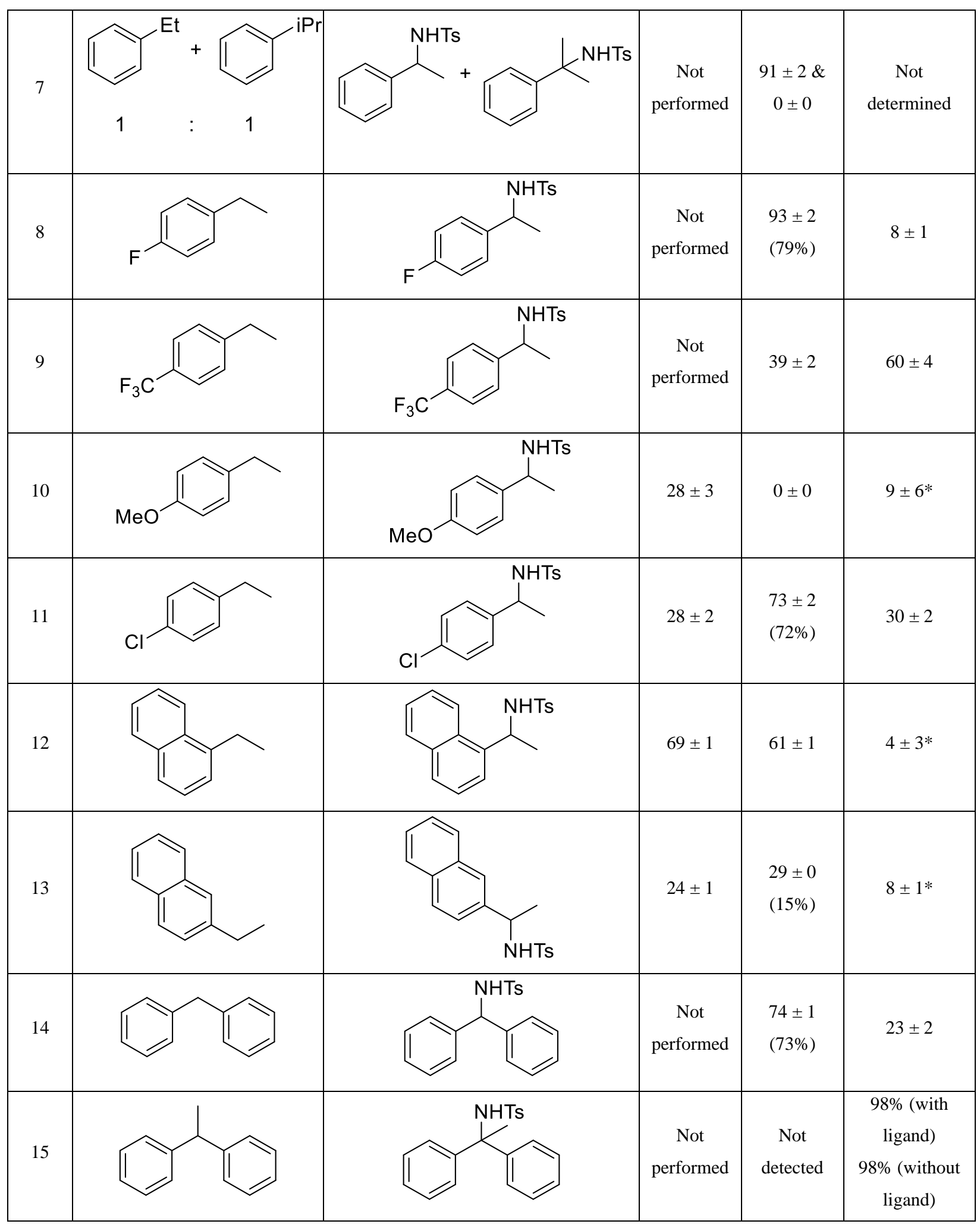




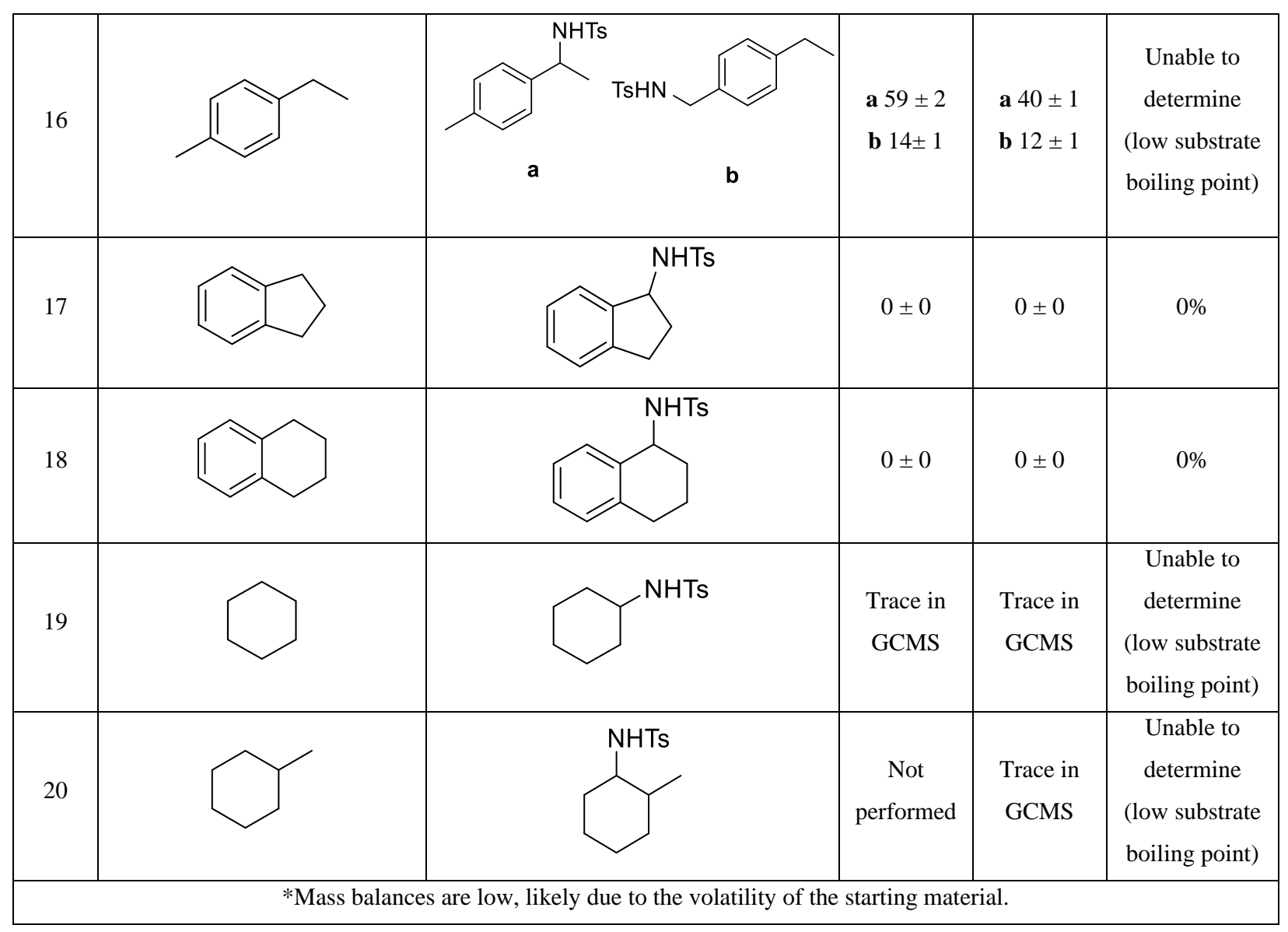




\section{Amine Scope}

Table S7. Amine Scope. Ethylbenzene ( $0.125 \mathrm{mmol}, 1.00$ equiv.), $\mathrm{Cu}\left(\mathrm{BF}_{4}\right)_{2} \cdot 6 \mathrm{H}_{2} \mathrm{O}(4.6 \mathrm{mg}, 12.6 \mu \mathrm{mol}, 10.0 \mathrm{~mol} \%)$, ligand A $(6.0 \mathrm{mg}, 12.6 \mu \mathrm{mol}, 10.0 \mathrm{~mol} \%)$, amine (p-Toluenesulfonamide, $64.2 \mathrm{mg}, 0.375 \mathrm{mmol}, 3.00$ equiv.), $\mathrm{N}$ Fluorobenzenesulfonimide (118.3 mg, $0.375 \mathrm{mmol}, 3.00$ equiv.) and $1.5 \mathrm{~mL} \mathrm{PhCF}_{3}, 48 \mathrm{~h}$. The reactions were prepared according to the Representative Procedure for Catalytic Benzylic Amination Reactions.

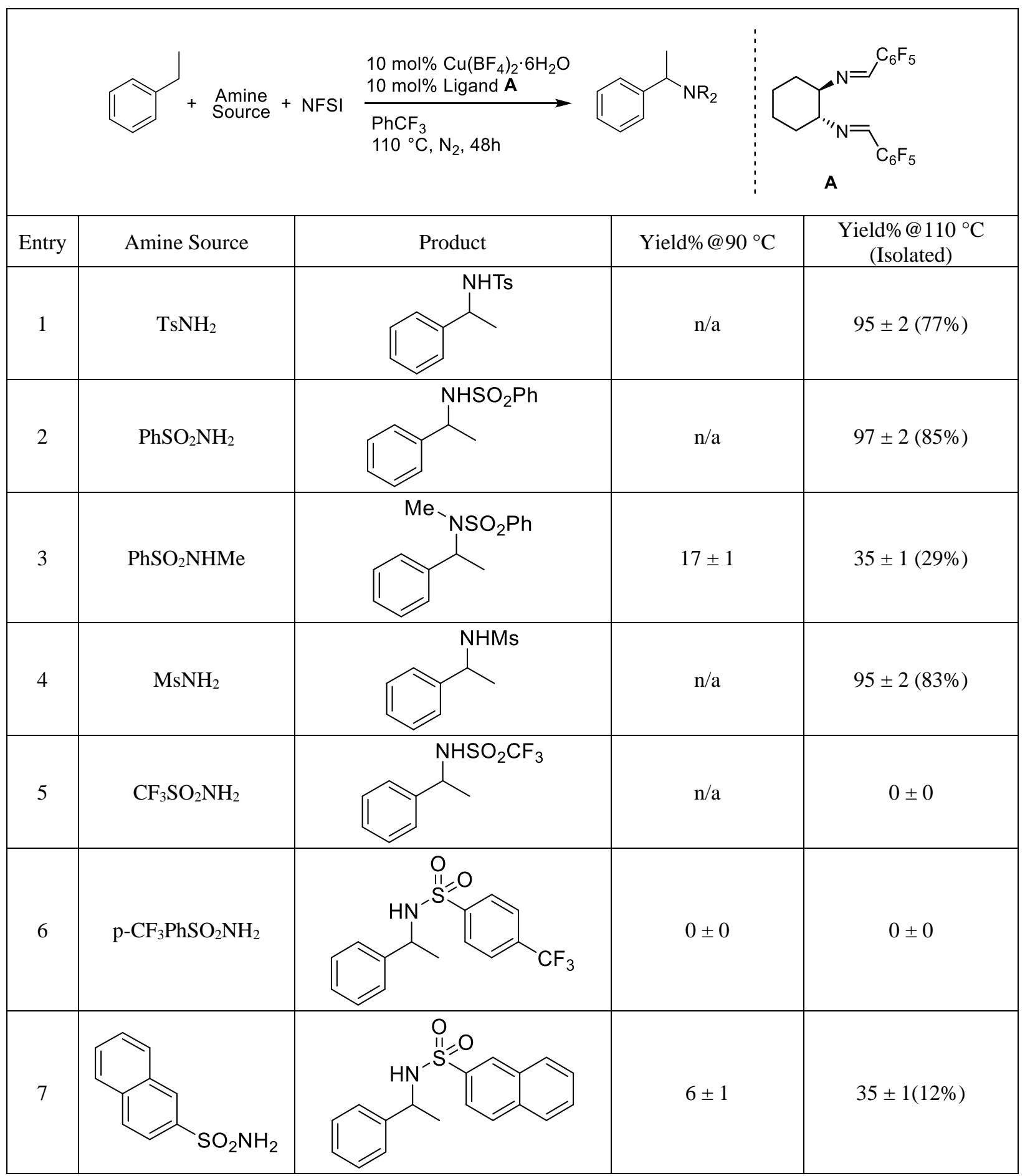




\begin{tabular}{|c|c|c|c|c|}
\hline 8 & p-MeO-PhSO ${ }_{2} \mathrm{NH}_{2}$ & 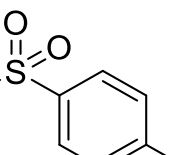 & $\mathrm{n} / \mathrm{a}$ & $16 \pm 1$ \\
\hline 9 & Urea & & $\mathrm{n} / \mathrm{a}$ & $0 \pm 0$ \\
\hline 10 & $\mathrm{PhCONH}_{2}$ & & $18 \pm 1$ & $29 \pm 2(22 \%)$ \\
\hline 11 & $\mathrm{C}_{6} \mathrm{~F}_{5} \mathrm{CONH}_{2}$ & & $18 \pm 1$ & $27 \pm 2(18 \%)$ \\
\hline 12 & $\mathrm{CH}_{3} \mathrm{CONH}_{2}$ & $\mathrm{NHCOCH}_{3}$ & $\mathrm{n} / \mathrm{a}$ & $\begin{array}{c}\text { Trace in GC-MS (But } \\
\text { difficult to quantify } \\
\text { by NMR due to low } \\
\text { quantity) }\end{array}$ \\
\hline 13 & $\mathrm{CF}_{3} \mathrm{CONH}_{2}$ & $\mathrm{NHCOCF}_{3}$ & $\mathrm{n} / \mathrm{a}$ & $0 \pm 0$ \\
\hline
\end{tabular}




\section{Study of Product Decomposition}

Table S8. Study of Product Decomposition. N-(1-phenylethyl)-p-toluenesulfonamide (34.4 mg, $0.125 \mathrm{mmol}, 1.00$ equiv.), $\mathrm{Cu}\left(\mathrm{BF}_{4}\right)_{2} \cdot 6 \mathrm{H}_{2} \mathrm{O}(4.6 \mathrm{mg}, 12.6 \mu \mathrm{mol}, 10.0 \mathrm{~mol} \%)$, ligand $\mathbf{A}(6.0 \mathrm{mg}, 12.6 \mu \mathrm{mol}, 10.0 \mathrm{~mol} \%)$, amination reagent 1 (p-Toluenesulfonamide, $64.2 \mathrm{mg}, 0.375 \mathrm{mmol}, 3.00$ equiv.), $N$-Fluorobenzenesulfonimide (118.3 $\mathrm{mg}$, $0.375 \mathrm{mmol}, 3.00$ equiv.) and $1.5 \mathrm{~mL} \mathrm{PhCF}_{3}, 48 \mathrm{~h}$. The reactions were prepared according to the Representative Procedure for Catalytic Benzylic Amination Reactions.

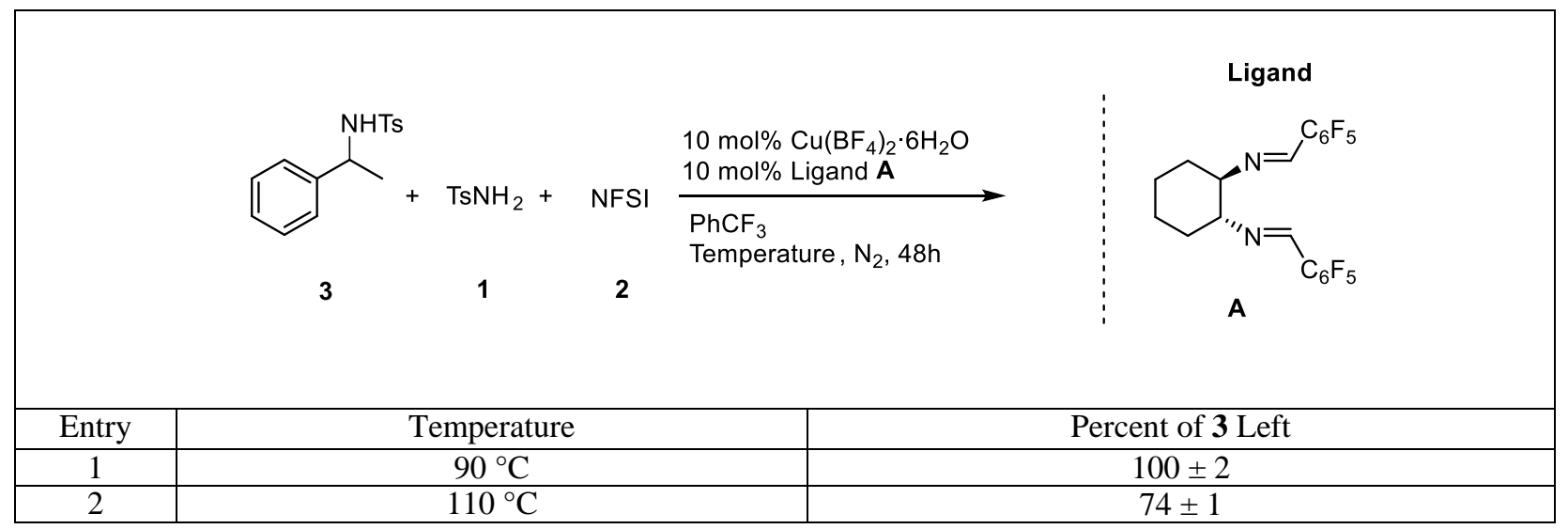

\section{Solvent Volume Study}

Table S9. Solvent Volume Study. Ethylbenzene ( $16.0 \mu \mathrm{L}, 13.3 \mathrm{mg}, 0.125 \mathrm{mmol}, 1.00$ equiv.), $\mathrm{Cu}\left(\mathrm{BF}_{4}\right)_{2} \cdot 6 \mathrm{H}_{2} \mathrm{O}(4.6$ $\mathrm{mg}, 12.6 \mu \mathrm{mol}, 10.0 \mathrm{~mol} \%)$, ligand $\mathbf{A}(6.0 \mathrm{mg}, 12.6 \mu \mathrm{mol}, 10.0 \mathrm{~mol} \%)$, amination reagent $\mathbf{1}$ (p-Toluenesulfonamide, $64.2 \mathrm{mg}, 0.375 \mathrm{mmol}, 3.00$ equiv.), $N$-Fluorobenzenesulfonimide (118.3 mg, $0.375 \mathrm{mmol}, 3.00 \mathrm{equiv}$.) and benzotrifluoride, $110^{\circ} \mathrm{C}, 48 \mathrm{~h}$. The reactions were prepared according to the Representative Procedure for Catalytic Benzylic Amination Reactions.

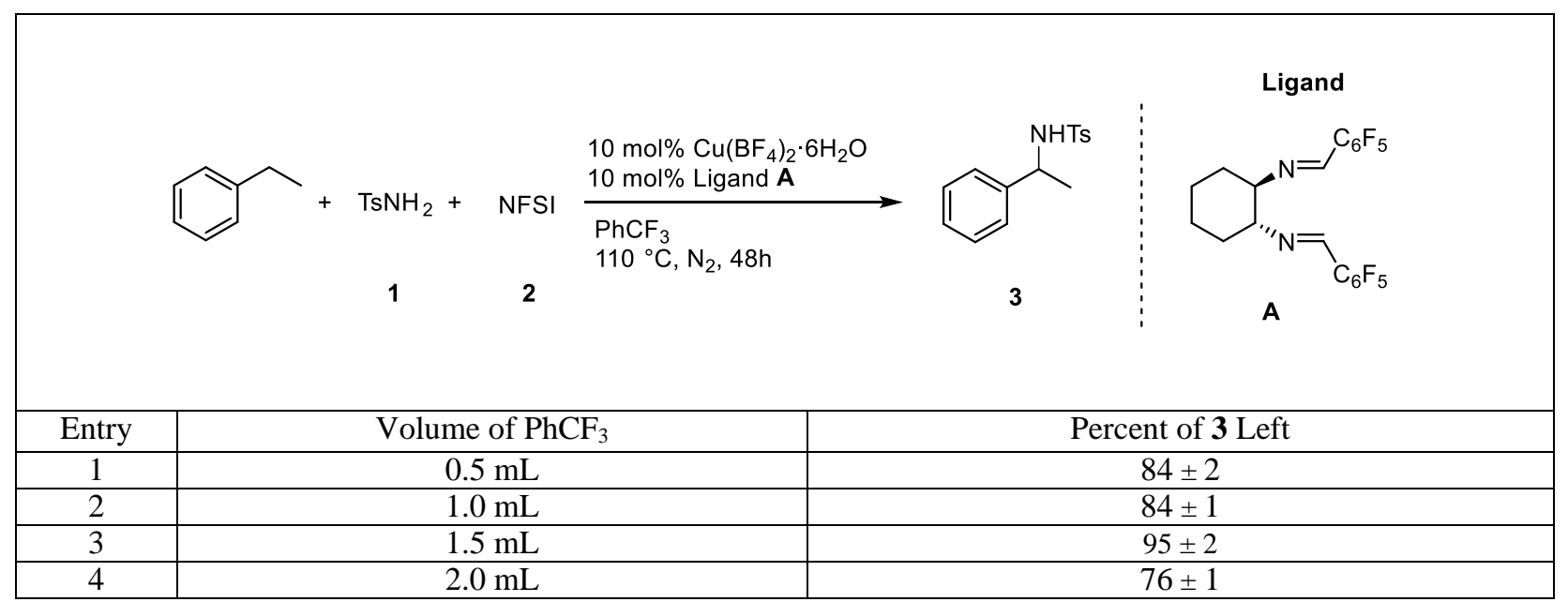




\section{Solvent Study for $\mathrm{PhCONH}_{2}$ Reactions}

Table S10. Solvent Study. Ethylbenzene ( $16.0 \mu \mathrm{L}, 13.3 \mathrm{mg}, 0.125 \mathrm{mmol}, 1.00$ equiv.), $\mathrm{Cu}\left(\mathrm{BF}_{4}\right)_{2} \cdot 6 \mathrm{H}_{2} \mathrm{O}(4.6 \mathrm{mg}$, $12.6 \mu \mathrm{mol}, 10.0 \mathrm{~mol} \%)$, ligand A (6.0 mg, $12.6 \mu \mathrm{mol}, 10.0 \mathrm{~mol} \%), \mathrm{PhCONH}_{2}(45.4 \mathrm{mg}, 0.375 \mathrm{mmol}, 3.00$ equiv.), $\mathrm{N}$-Fluorobenzenesulfonimide ( $118.3 \mathrm{mg}, 0.375 \mathrm{mmol}, 3.00$ equiv.) and $1.5 \mathrm{~mL}$ solvent, $110{ }^{\circ} \mathrm{C}, 48 \mathrm{~h}$. The reactions were prepared according to the Representative Procedure for Catalytic Benzylic Amination Reactions.

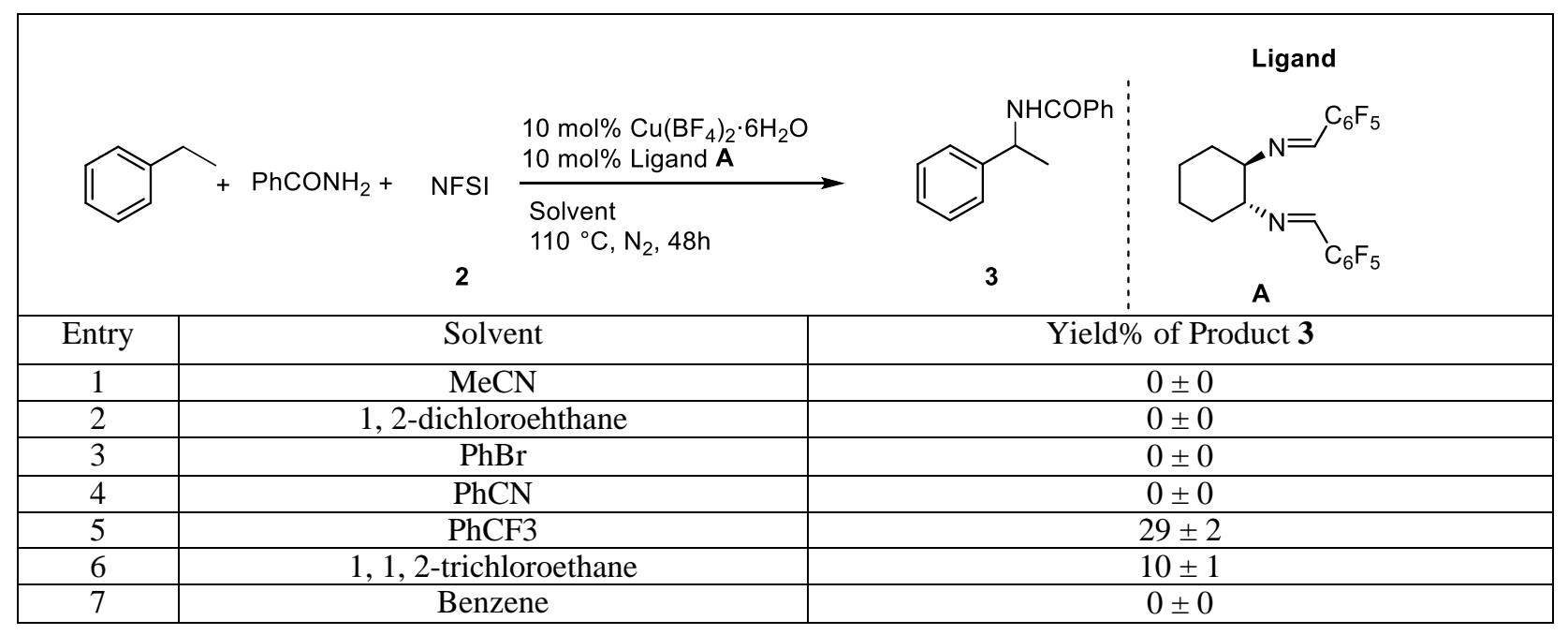




\section{Optimization of Amine and NFSI for $\mathrm{PhCONH}_{2}$ Reactions}

Table S11. Optimization of Amine and NFSI. Ethylbenzene (16.0 $\mu \mathrm{L}, 13.3 \mathrm{mg}, 0.125 \mathrm{mmol}, 1.00$ equiv.), $\mathrm{Cu}\left(\mathrm{BF}_{4}\right)_{2} \cdot 6 \mathrm{H}_{2} \mathrm{O}$ (4.6 mg, $\left.12.6 \mu \mathrm{mol}, 10.0 \mathrm{~mol} \%\right)$, ligand $(12.6 \mu \mathrm{mol}, 10.0 \mathrm{~mol} \%), \mathrm{PhCONH}_{2}, N-$ Fluorobenzenesulfonimide and $1.5 \mathrm{~mL}$ benzotrifluoride $110{ }^{\circ} \mathrm{C}, 48 \mathrm{~h}$. The reactions were prepared according to the Representative Procedure for Catalytic Benzylic Amination Reactions.

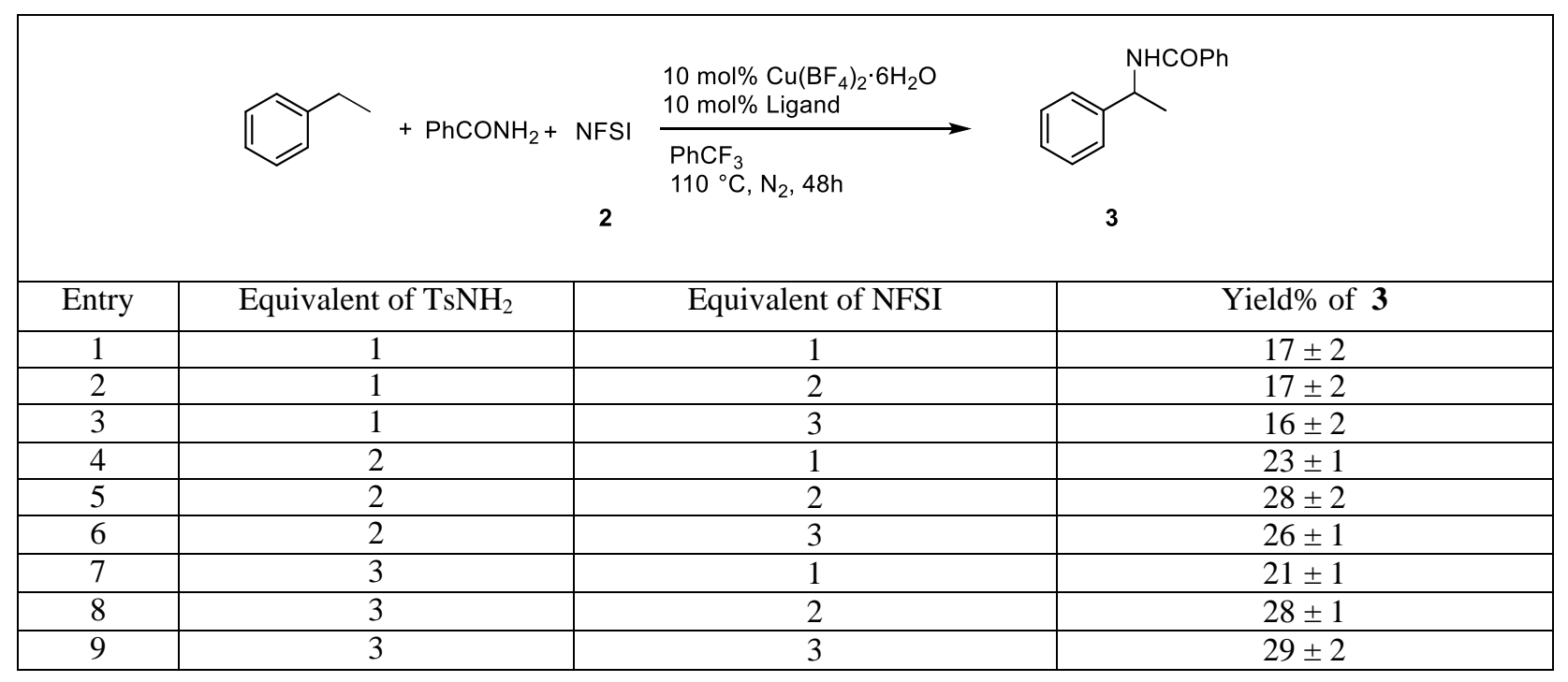




\section{Synthesis of Substrate \\ Ethane-1,1-diyldibenzene}

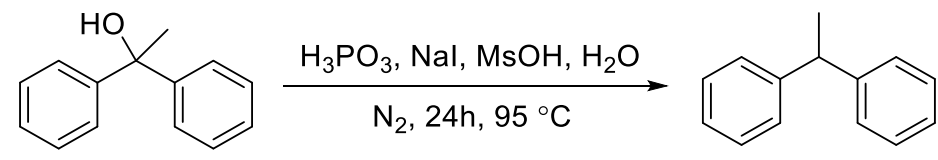

The title compound was synthesized in analogy to a literature known procedure ${ }^{1}$.

A $20 \mathrm{~mL}$ scintillation vial equipped with a Teflon-coated stir bar was charged with 1,1diphenylethanol ( $0.91 \mathrm{~g}, 5.0 \mathrm{mmol}, 1.0$ equiv.), sodium iodide (225 mg, $1.5 \mathrm{mmol}, 0.3$ equiv.), and concentrated phosphoric acid (615 mg, $7.5 \mathrm{mmol}, 1.5$ equiv.). The vial was flushed with $\mathrm{N}_{2}$ and charged with $1.7 \mathrm{~mL}$ of $\mathrm{H}_{2} \mathrm{O}$ and $3.3 \mathrm{~mL}$ of methanesulfonic acid. The reaction mixture was heated to $95{ }^{\circ} \mathrm{C}$ for $24 \mathrm{~h}$. Upon completion, the reaction was cooled to room temperature and washed with $10 \mathrm{~mL} \mathrm{H}_{2} \mathrm{O}$. The aqueous layer was extracted with EtOAc $(2 \times 15 \mathrm{~mL})$ and the organic layers were combined. The solvent of the mixture was removed under reduced pressure to afford the crude product, which was purified on silica using hexane as eluent to afford the product as a colorless oil (630 $\mathrm{mg}, 68 \%)$.

${ }^{1} \mathrm{H}$ NMR $\left(500 \mathrm{MHz}, \mathrm{CDCl}_{3}, 25^{\circ} \mathrm{C}\right): \delta[\mathrm{ppm}]=7.44-7.28(\mathrm{~m}, 10 \mathrm{H} ; \mathrm{Ar}-\mathrm{H}), 4.28(\mathrm{q}, 1 \mathrm{H})$, $1.78(\mathrm{~d}, 3 \mathrm{H})$

${ }^{13} \mathrm{C}\left(\mathrm{NMR} 125 \mathrm{MHz}, \mathrm{CDCl}_{3}, 25^{\circ} \mathrm{C}\right): \delta[\mathrm{ppm}]=146.5(\mathrm{~s}), 128.5(\mathrm{~s}), 127.8(\mathrm{~s}), 126.1(\mathrm{~s})$, $44.9(\mathrm{~s}), 22.0(\mathrm{~s})$; 


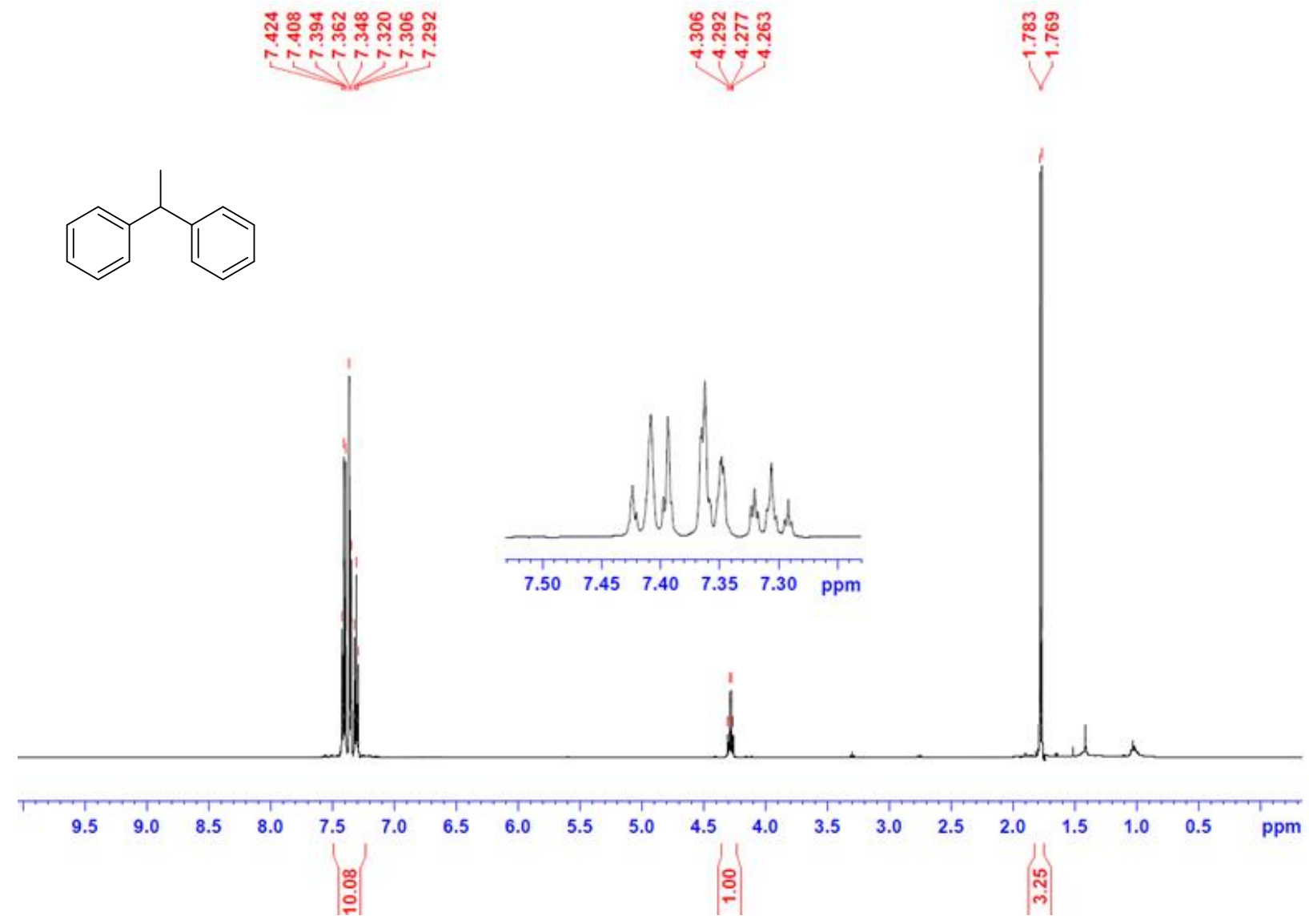

Figure S2. ${ }^{1} \mathrm{H}$ NMR of Ethane-1,1-diyldibenzene in $\mathrm{CDCl}_{3}$. 


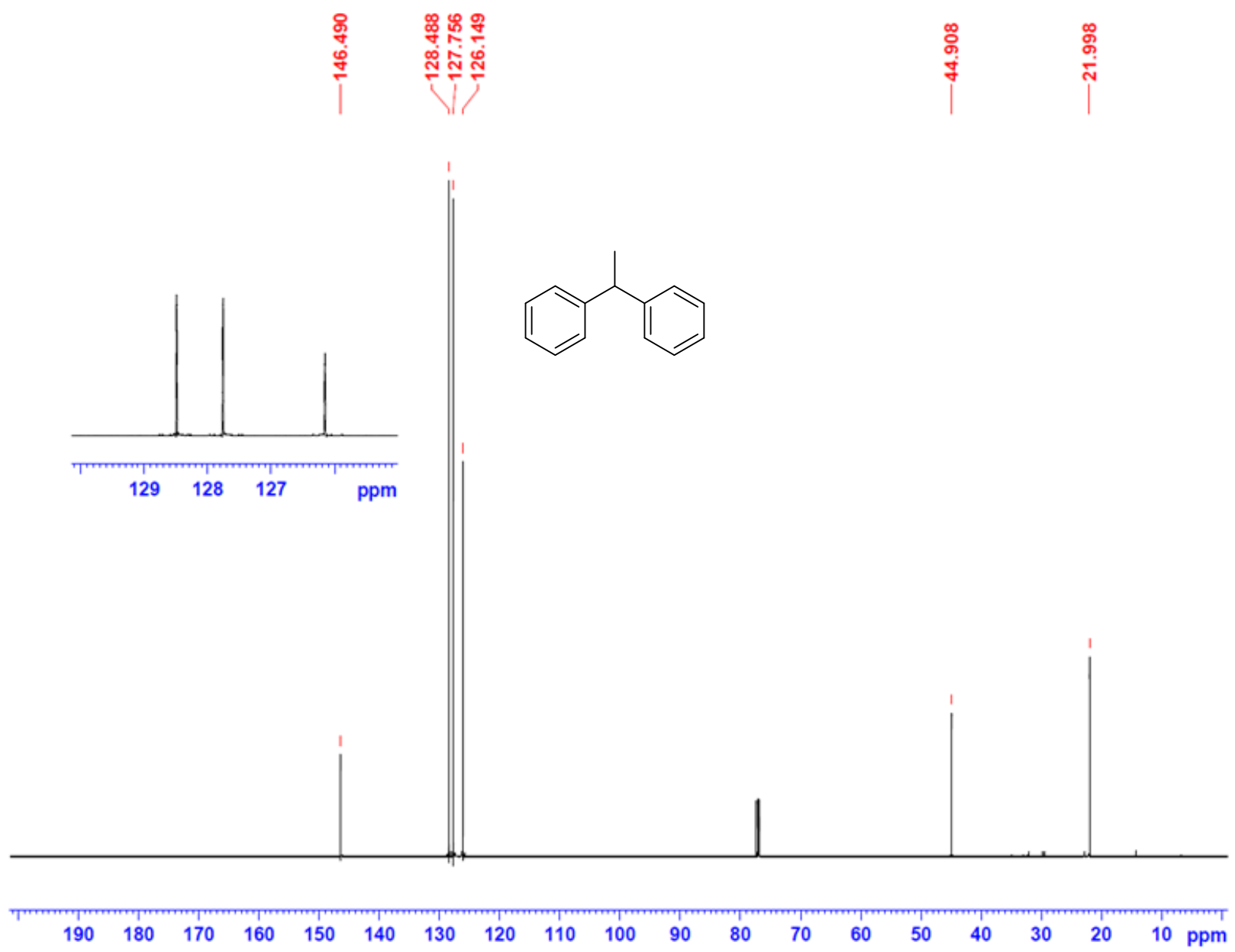

Figure S3. ${ }^{13} \mathrm{C}$ NMR of Ethane-1,1-diyldibenzene in $\mathrm{CDCl}_{3}$. 


\section{Isolation of Selected Products from Catalytic Reactions}

\section{4-Methyl-N-(1-phenylethyl)benzenesulfonamide}<smiles>CNC(C)c1ccccc1</smiles>

In a glovebox, $\mathrm{Cu}\left(\mathrm{BF}_{4}\right)_{2} \cdot 6 \mathrm{H}_{2} \mathrm{O}(23.0 \mathrm{mg}, 63.0 \mu \mathrm{mol}, 10.0 \mathrm{~mol} \%)$, ligand $\mathbf{A}(30.0 \mathrm{mg}, 63 \mu \mathrm{mol}$, $10.0 \mathrm{~mol} \%$ ), $\mathrm{TsNH}_{2}$ (324 mg, $1.88 \mathrm{mmol}, 3.00$ equiv), $N$-Fluorobenzenesulfonimide (593 mg, $1.88 \mathrm{mmol}, 3.00$ equiv) and ethylbenzene (78 $\mu \mathrm{L}, 66.4 \mathrm{mg}, 0.625 \mathrm{mmol}, 1.00$ equiv.) were added in this sequence to a $20 \mathrm{~mL}$ scintillation vial equipped with a Teflon-coated stir bar. The vial was sealed with a Teflon-lined vial cap and heated to $110^{\circ} \mathrm{C}$ on a pre-heated vial plate under vigorous stirring (1500 rpm). After $48 \mathrm{~h}$, the vial was taken off the heating block and the mixture was allowed to cool to room temperature. The solvent was removed under reduced pressure. 15 $\mathrm{mL} \mathrm{CHCl}_{3}$ and $2 \mathrm{~g} \mathrm{CaCO}_{3}$ was added to mixture, which was then filtered through Celite. The filtrate was concentrated under vacuum (rotary evaporator) to yield the crude product. Purification was performed by column chromatography on silica using ethyl acetate/hexane (1:4) as eluent. $133 \mathrm{mg}$ (77\% yield) of pure title compound was obtained. The ${ }^{1} \mathrm{H}$ and ${ }^{13} \mathrm{C}$ NMR match the reported spectra. ${ }^{2}$

${ }^{1} \mathrm{H}$ NMR $\left(500 \mathrm{MHz}, \mathrm{CDCl}_{3}, 25^{\circ} \mathrm{C}\right): \delta[\mathrm{ppm}]=7.76(\mathrm{~d}, 2 \mathrm{H} ; \mathrm{Ar}-\mathrm{H}), 7.30(\mathrm{~d}, 2 \mathrm{H} ; \mathrm{Ar}-\mathrm{H})$, 7.17-7.38 (m, 5H; Ar-H), 4.79 (br t, $1 \mathrm{H} ; \mathrm{NH}), 4.11$ (d, 2H; - $\left.\underline{\mathrm{C}} \underline{\mathrm{H}_{2}} \mathrm{Ph}\right), 2.43\left(\mathrm{~s}, 3 \mathrm{H} ; \mathrm{C}_{3} 3 \mathrm{PhSO}_{2}-\right.$ );

${ }^{13} \mathrm{C}\left(\mathrm{NMR} 125 \mathrm{MHz}, \mathrm{CDCl}_{3}, 25^{\circ} \mathrm{C}\right): \delta[\mathrm{ppm}]=143.6(\mathrm{~s}), 137.0(\mathrm{~s}), 136.4(\mathrm{~s}), 129.9(\mathrm{~s})$, 128.8 (s), 128.0 (s), 128.0 (s), 127.3 (s), 47.4 (s), 21.7 (s); 


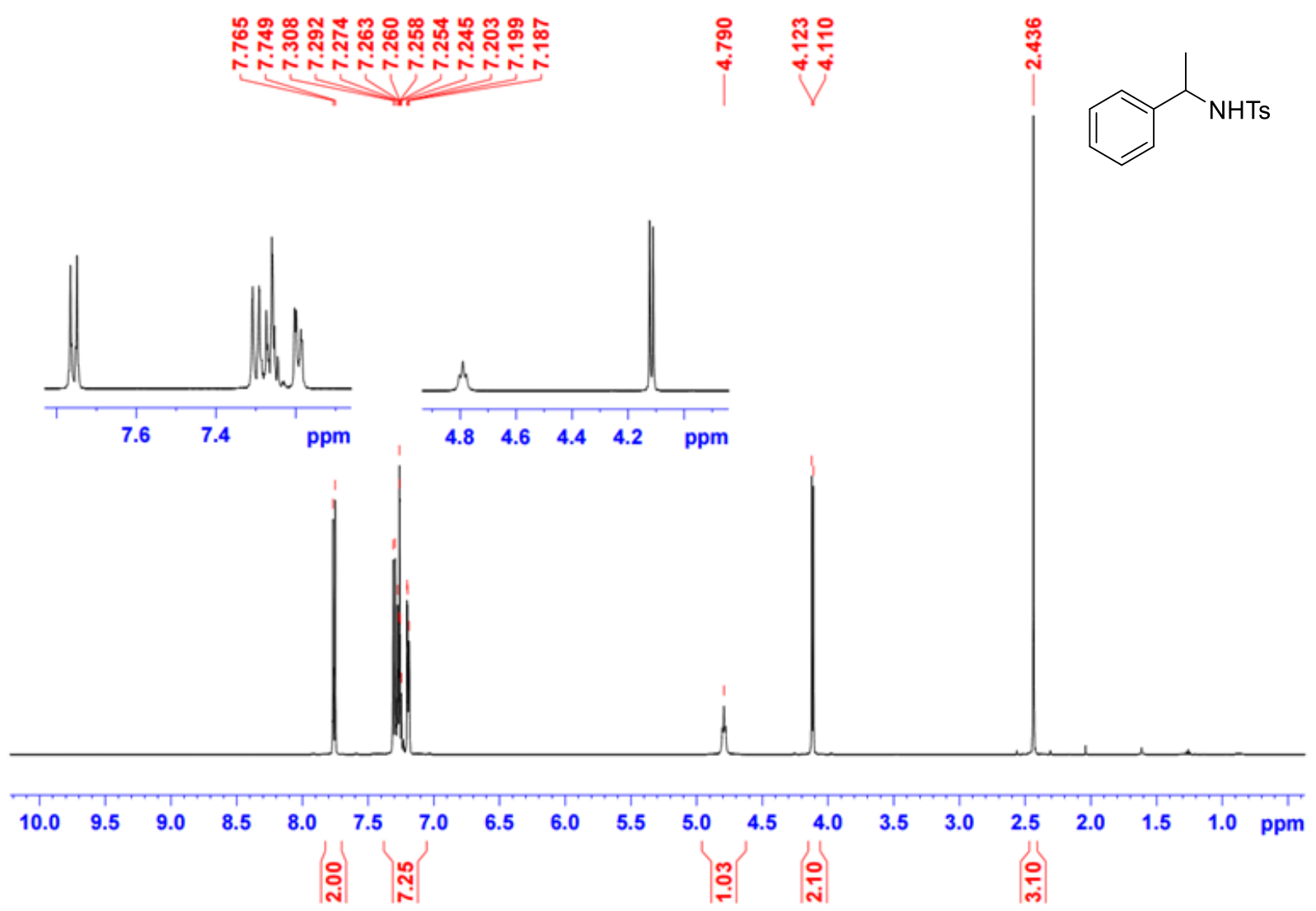

Figure $\mathrm{S4} .{ }^{1} \mathrm{H}$ NMR of $\mathrm{N}$-Benzyl-4-methylbenzenesulfonamide in $\mathrm{CDCl}_{3}$. 


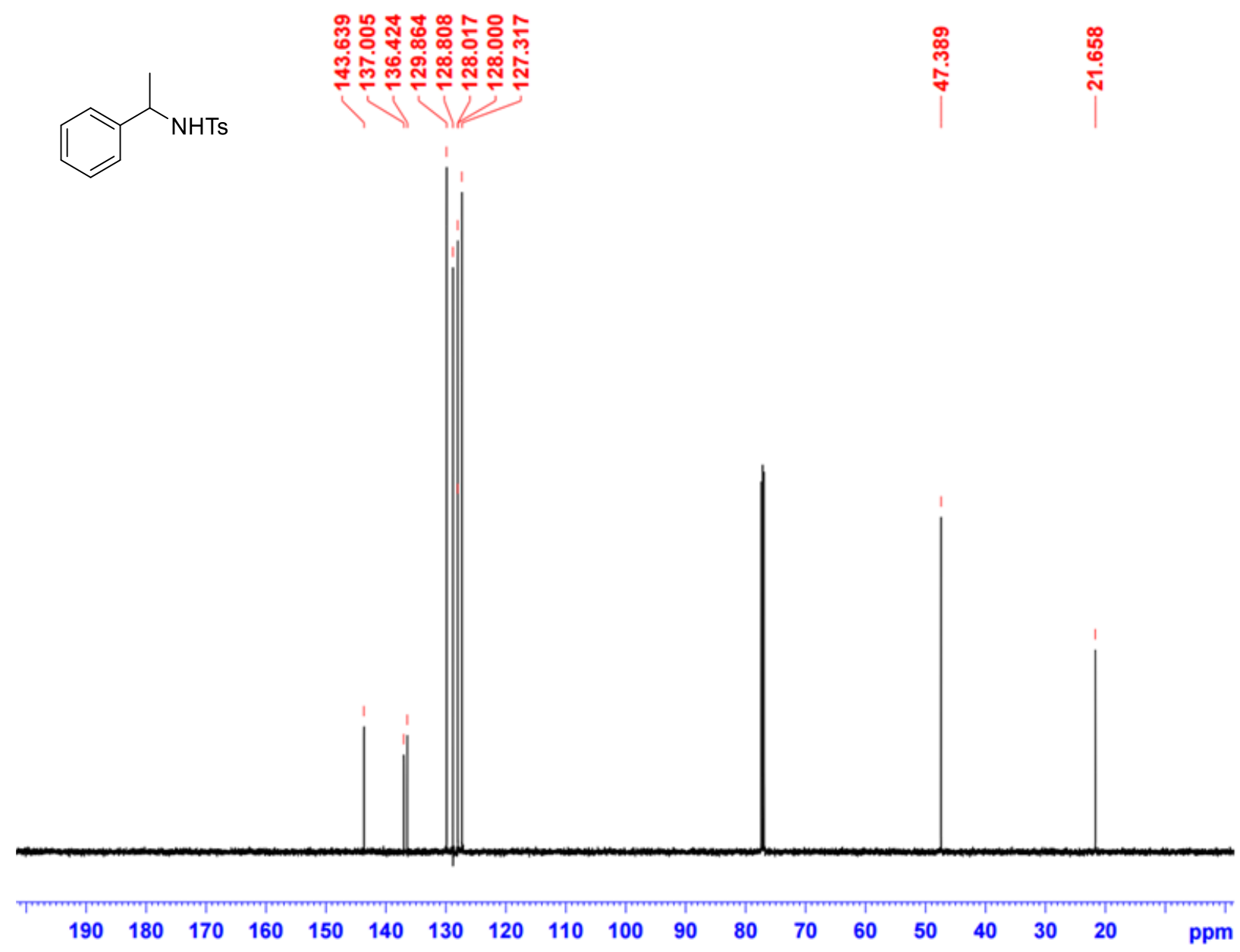

Figure S5. ${ }^{13} \mathrm{C}$ NMR of $N$-Benzyl-4-methylbenzenesulfonamide in $\mathrm{CDCl}_{3}$. 


\section{N-(1-(4-Fluorophenyl)ethyl)-4-methylbenzenesulfonamide}<smiles>CNC(C)c1ccc(F)cc1</smiles>

In a glovebox, $\mathrm{Cu}\left(\mathrm{BF}_{4}\right)_{2} \cdot 6 \mathrm{H}_{2} \mathrm{O}(23.0 \mathrm{mg}, 63.0 \mu \mathrm{mol}, 10.0 \mathrm{~mol} \%)$, ligand $\mathbf{A}(30.0 \mathrm{mg}, 63 \mu \mathrm{mol}$, $10.0 \mathrm{~mol} \%$ ), $\mathrm{TsNH}_{2}$ (324 mg, $1.88 \mathrm{mmol}, 3.00$ equiv), $N$-Fluorobenzenesulfonimide (593 mg, $1.88 \mathrm{mmol}, 3.00$ equiv) and 1-ethyl-4-fluorobenzene ( $79 \mu \mathrm{L}, 77.6 \mathrm{mg}, 0.625 \mathrm{mmol}, 1.00 \mathrm{equiv}$. were added in this sequence to a $20 \mathrm{~mL}$ scintillation vial equipped with a Teflon-coated stir bar. The vial was sealed with a Teflon-lined vial cap and heated to $110^{\circ} \mathrm{C}$ on a pre-heated vial plate under vigorous stirring $(1500 \mathrm{rpm})$. After $48 \mathrm{~h}$, the vial was taken off the heating block and the mixture was allowed to cool to room temperature. The solvent was removed under reduced pressure. $15 \mathrm{~mL} \mathrm{CHCl}_{3}$ and $2 \mathrm{~g} \mathrm{CaCO}_{3}$ was added into the mixture, which was then filtered through a Celite plug. The filtrate was concentrated under vacuum (rotary evaporator) to yield the crude product which was purified by column chromatography using ethyl acetate/hexane (1:4) as eluent. $145 \mathrm{mg}$ (79\% yield) of pure title compound was obtained. The ${ }^{1} \mathrm{H}$ and ${ }^{13} \mathrm{C} \mathrm{NMR}$ match the reported spectra. ${ }^{2}$

${ }^{1} \mathrm{H}$ NMR $\left(500 \mathrm{MHz}, \mathrm{CDCl}_{3}, 25^{\circ} \mathrm{C}\right): \delta[\mathrm{ppm}]=7.60(2 \mathrm{H}, \mathrm{d}), 7.16(2 \mathrm{H}, \mathrm{d}), 7.07(2 \mathrm{H}, \mathrm{m})$, $6.83(2 \mathrm{H}, \mathrm{m}), 5.57(1 \mathrm{H}, \mathrm{d}, \mathrm{NH}), 4.44(1 \mathrm{H}$, quintet $), 2.37(3 \mathrm{H}, \mathrm{s}), 1.36(3 \mathrm{H}, \mathrm{d})$;

${ }^{13} \mathrm{C}\left(\mathrm{NMR} 125 \mathrm{MHz}, \mathrm{CDCl}_{3}, 25^{\circ} \mathrm{C}\right): \delta[\mathrm{ppm}]=163.0(\mathrm{~s}), 161.0(\mathrm{~s}), 143.3(\mathrm{~s}), 138.1(\mathrm{~d})$, 137.7 (s), 129.5 (s), 127.9 (d), 127.1 (s), 115.3 (d), 53.1 (s), 23.6 (s), 21.5 (s); 


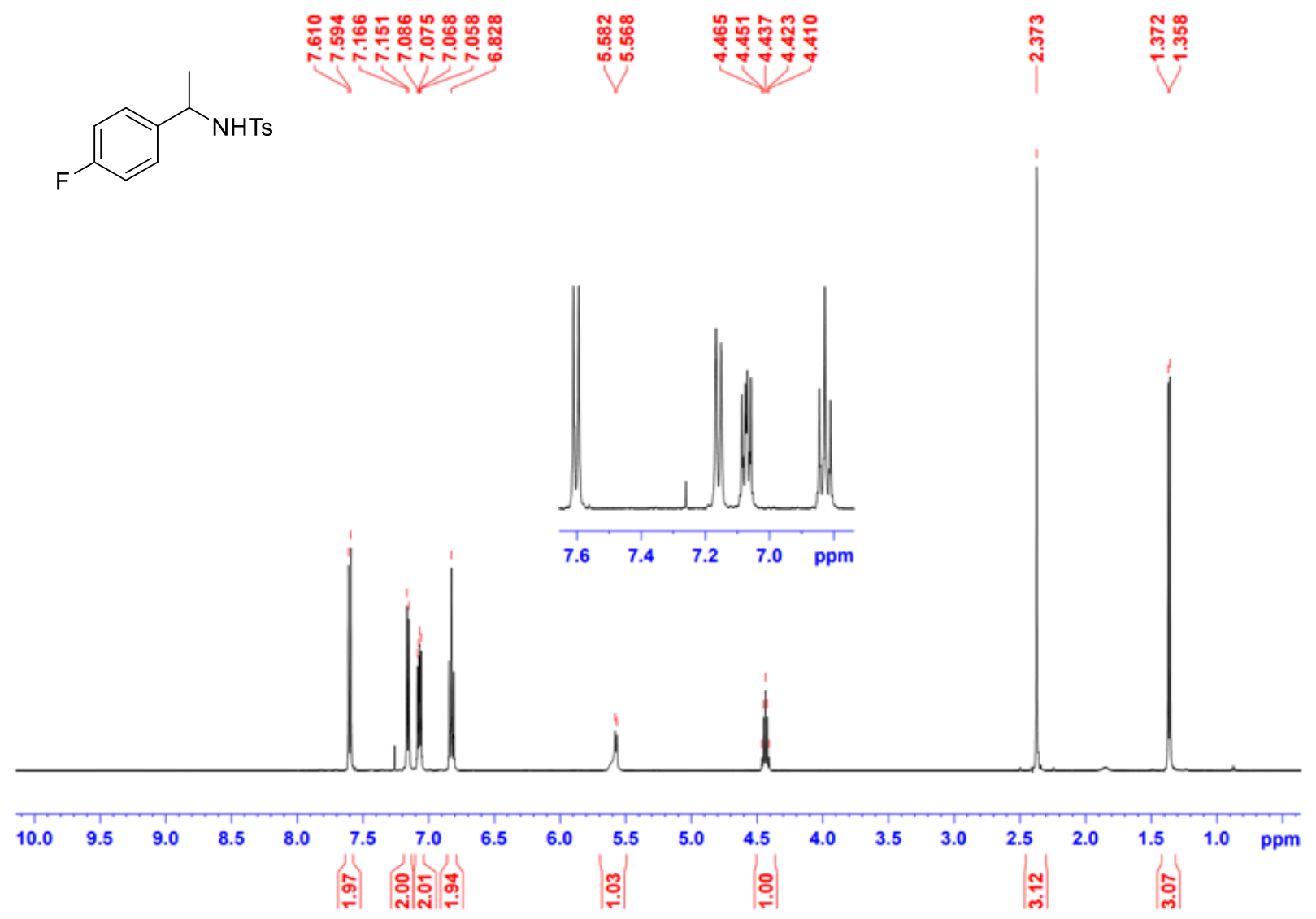

Figure S6. ${ }^{1} \mathrm{H}$ NMR of N-(1-(4-Fluorophenyl)ethyl)-4-methylbenzenesulfonamide in $\mathrm{CDCl}_{3}$. 

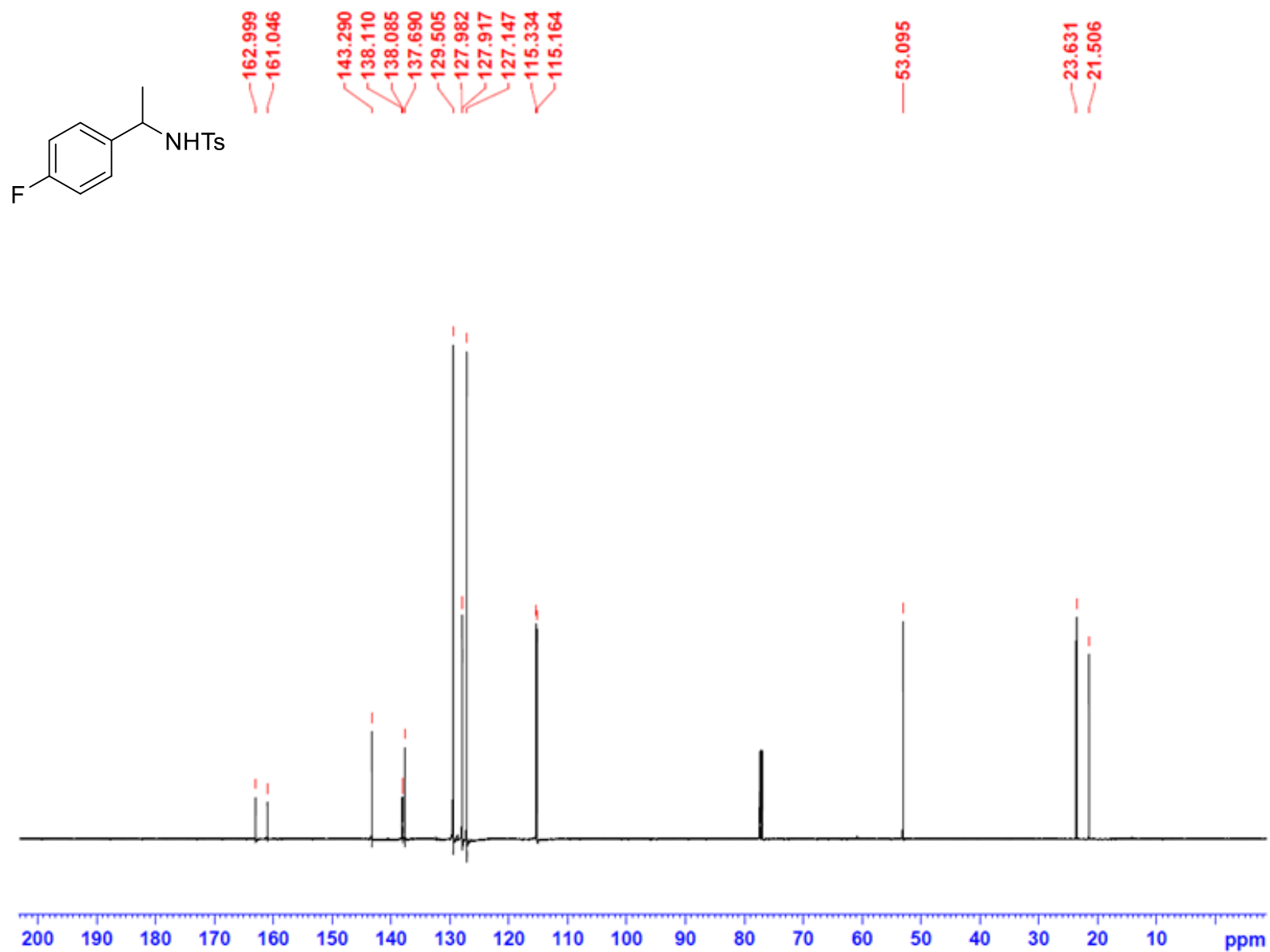

Figure S7. ${ }^{13} \mathrm{C}$ NMR of N-(1-(4-Fluorophenyl)ethyl)-4-methylbenzenesulfonamide in $\mathrm{CDCl}_{3}$. 


\section{N-(1-(4-Chlorophenyl)ethyl)-4-methylbenzenesulfonamide}<smiles>CNC(C)c1ccc(Cl)cc1</smiles>

In a glovebox, $\mathrm{Cu}\left(\mathrm{BF}_{4}\right)_{2} \cdot 6 \mathrm{H}_{2} \mathrm{O}(23.0 \mathrm{mg}, 63.0 \mu \mathrm{mol}, 10.0 \mathrm{~mol} \%)$, ligand $\mathbf{A}(30.0 \mathrm{mg}, 63 \mu \mathrm{mol}$, $10.0 \mathrm{~mol} \%$ ), $\mathrm{TsNH}_{2}$ (324 mg, $1.88 \mathrm{mmol}, 3.00$ equiv), $N$-Fluorobenzenesulfonimide (593 mg, $1.88 \mathrm{mmol}, 3.00$ equiv) and 1-ethyl-4-chlorobenzene ( $90 \mu \mathrm{L}, 87.9 \mathrm{mg}, 0.625 \mathrm{mmol}, 1.00$ equiv.) were added in this sequence to a $20 \mathrm{~mL}$ scintillation vial equipped with a Teflon-coated stir bar. The vial was sealed with a Teflon-lined vial cap and heated to $110^{\circ} \mathrm{C}$ on a pre-heated vial plate under vigorous stirring $(1500 \mathrm{rpm})$. After $48 \mathrm{~h}$, the vial was taken off the heating block and the mixture was allowed to cool to room temperature. The solvent was removed under reduced pressure. $15 \mathrm{~mL} \mathrm{CHCl}_{3}$ and $2 \mathrm{~g} \mathrm{CaCO}_{3}$ were added into the mixture and the resulting suspension was filtered through Celite. The filtrate was concentrated under vacuum (rotary evaporator) to yield the crude product which was purified by column chromatography using ethyl acetate/hexane (1:4) as eluent. $139 \mathrm{mg}$ (72\% yield) of pure title compound was obtained. The ${ }^{1} \mathrm{H}$ and ${ }^{13} \mathrm{C}$ NMR match the literature reported spectra. ${ }^{2}$

${ }^{1} \mathrm{H}$ NMR $\left(500 \mathrm{MHz}, \mathrm{CDCl}_{3}, 25{ }^{\circ} \mathrm{C}\right): \delta[\mathrm{ppm}]=7.58(2 \mathrm{H}, \mathrm{d}), 7.14(2 \mathrm{H}, \mathrm{d}), 7.09(2 \mathrm{H}, \mathrm{d})$, $7.03(2 \mathrm{H}, \mathrm{d}), 5.76(1 \mathrm{H}, \mathrm{d}, \mathrm{NH}), 4.42$ (1H, quintet), $2.38(3 \mathrm{H}, \mathrm{s}), 1.35(3 \mathrm{H}, \mathrm{d})$;

${ }^{13} \mathrm{C}\left(\mathrm{NMR} 125 \mathrm{MHz}, \mathrm{CDCl}_{3}, 25^{\circ} \mathrm{C}\right): \delta[\mathrm{ppm}]=143.3(\mathrm{~s}), 140.8(\mathrm{~s}), 137.5(\mathrm{~s}), 133.0(\mathrm{~s})$, 129.5 (s), 128.5 (s), 127.7 (s), 127.1 (s), 53.1 (s), 23.4 (s), 21.5 (s); 


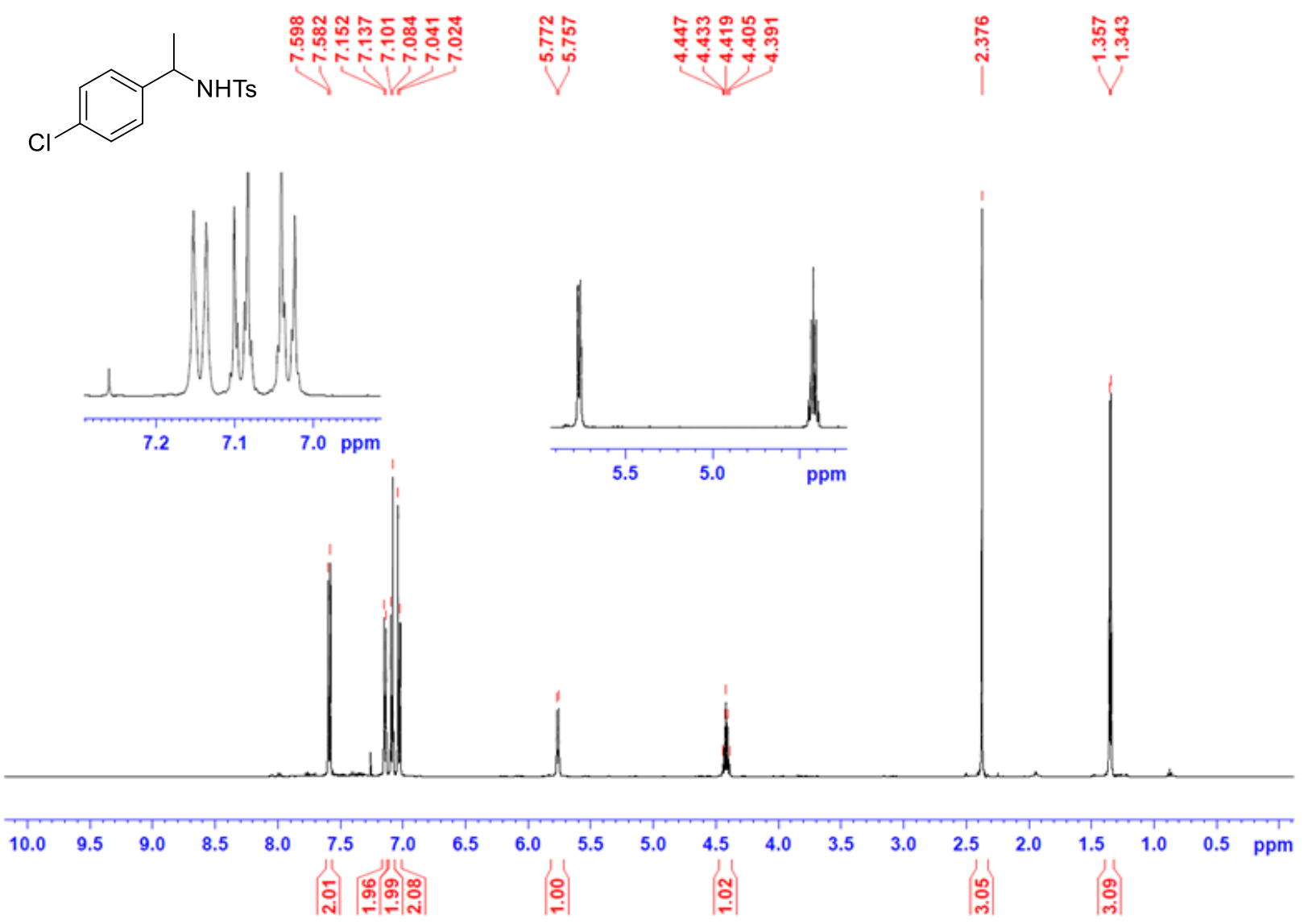

Figure S8. ${ }^{1} \mathrm{H}$ NMR of N-(1-(4-Chlorophenyl)ethyl)-4-methylbenzenesulfonamide in $\mathrm{CDCl}_{3}$. 

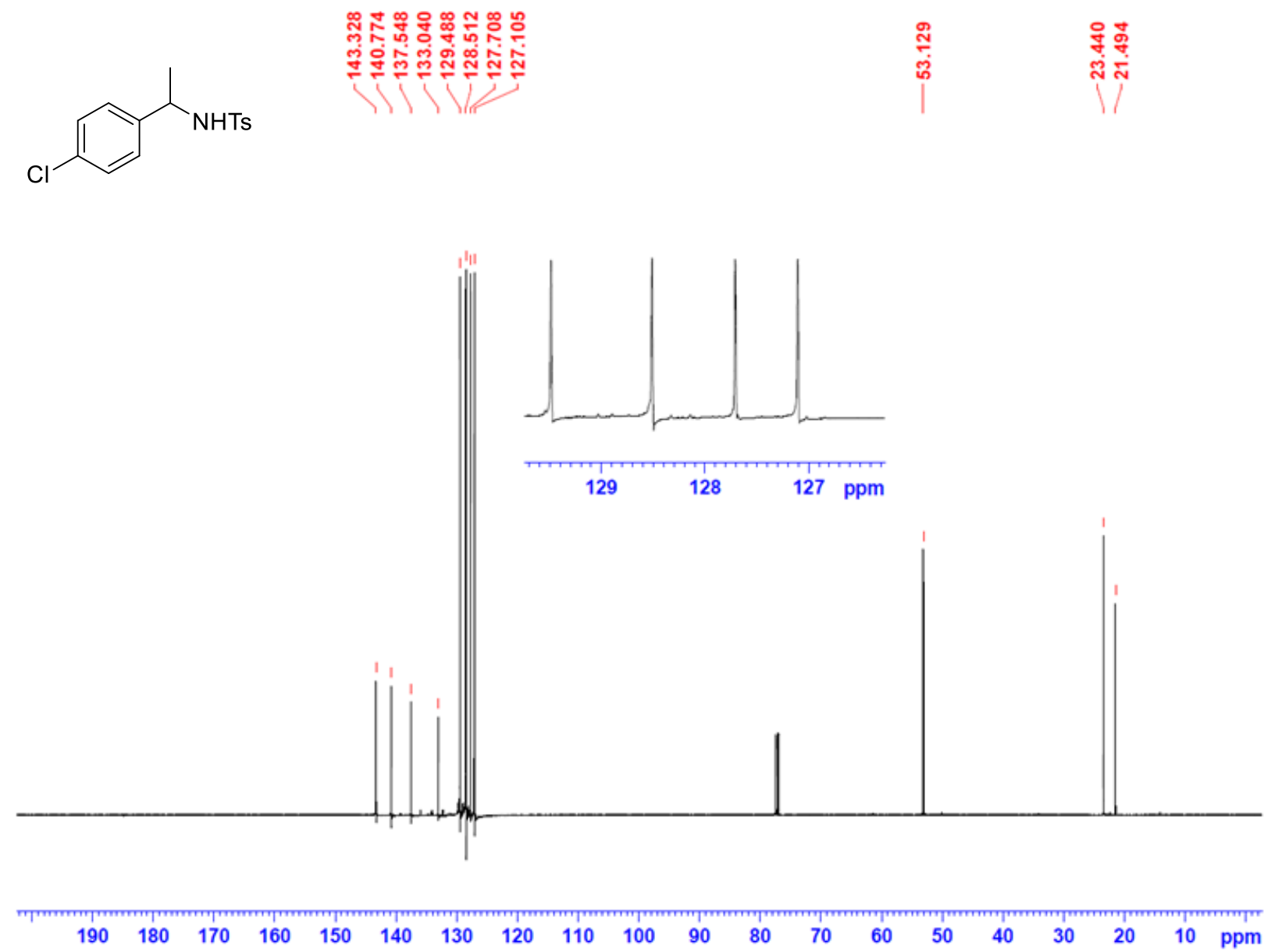

Figure S9. ${ }^{13} \mathrm{C}$ NMR of N-(1-(4-Chlorophenyl)ethyl)-4-methylbenzenesulfonamide in $\mathrm{CDCl}_{3}$. 


\section{4-Methyl-N-(1-phenylpropyl)benzenesulfonamide}<smiles>CCC([NH3+])c1ccccc1</smiles>

In a glovebox, $\mathrm{Cu}\left(\mathrm{BF}_{4}\right)_{2} \cdot 6 \mathrm{H}_{2} \mathrm{O}(23.0 \mathrm{mg}, 63.0 \mu \mathrm{mol}, 10.0 \mathrm{~mol} \%)$, ligand $\mathbf{A}(30.0 \mathrm{mg}, 63 \mu \mathrm{mol}$, $10.0 \mathrm{~mol} \%$ ), $\mathrm{TsNH}_{2}$ (324 mg, $1.88 \mathrm{mmol}, 3.00$ equiv), $\mathrm{N}$-Fluorobenzenesulfonimide (593 mg, $1.88 \mathrm{mmol}, 3.00$ equiv) and n-propylbenzene ( $88 \mu \mathrm{L}, 75.1 \mathrm{mg}, 0.625 \mathrm{mmol}, 1.00$ equiv.) were added in this sequence to a $20 \mathrm{~mL}$ scintillation vial equipped with a Teflon-coated stir bar. The vial was sealed with a Teflon-lined vial cap and heated to $110{ }^{\circ} \mathrm{C}$ on a pre-heated vial plate under vigorous stirring $(1500 \mathrm{rpm})$. After $48 \mathrm{~h}$, the vial was taken off the heating block and the mixture was allowed to cool to room temperature. The solvent was removed under reduced pressure. $15 \mathrm{~mL} \mathrm{CHCl}_{3}$ and $2 \mathrm{~g} \mathrm{CaCO}_{3}$ were added into the mixture and the resulting suspension was filtered through Celite. The filtrate was concentrated under vacuum (rotary evaporator) to yield the crude product which was purified by column chromatography using ethyl acetate/hexane (1:4) as eluent. $41 \mathrm{mg}$ (23\% yield) of pure title compound was obtained. The ${ }^{1} \mathrm{H}$ and ${ }^{13} \mathrm{C}$ NMR match the literature reported spectra. ${ }^{3}$

${ }^{1} \mathrm{H}$ NMR $\left(500 \mathrm{MHz}, \mathrm{CDCl}_{3}, 25^{\circ} \mathrm{C}\right): \delta[\mathrm{ppm}]=\delta 7.53(\mathrm{~d}, 2 \mathrm{H}), 7.18-7.13(\mathrm{~m}, 3 \mathrm{H}), 7.11$ (d, 2 H), 7.03-6.97 (m, 2 H), 4.74 (d, 1 H), 4.19 (q, 1 H), 2.35 (s, 3 H), 1.87-1.65 (m, 2 H), 0.78 (t, $3 \mathrm{H})$;

${ }^{13} \mathrm{C}\left(\mathrm{NMR} 125 \mathrm{MHz}, \mathrm{CDCl}_{3}, 25{ }^{\circ} \mathrm{C}\right): \delta[\mathrm{ppm}]=143.1(\mathrm{~s}), 140.8(\mathrm{~s}), 137.9(\mathrm{~s}), 129.4(\mathrm{~s})$, 128.6 (s), 127.5 (s), 127.2 (s), 126.7 (s), 60.0 (s), 30.7 (s), 21.6 (s), 10.6 (s); 


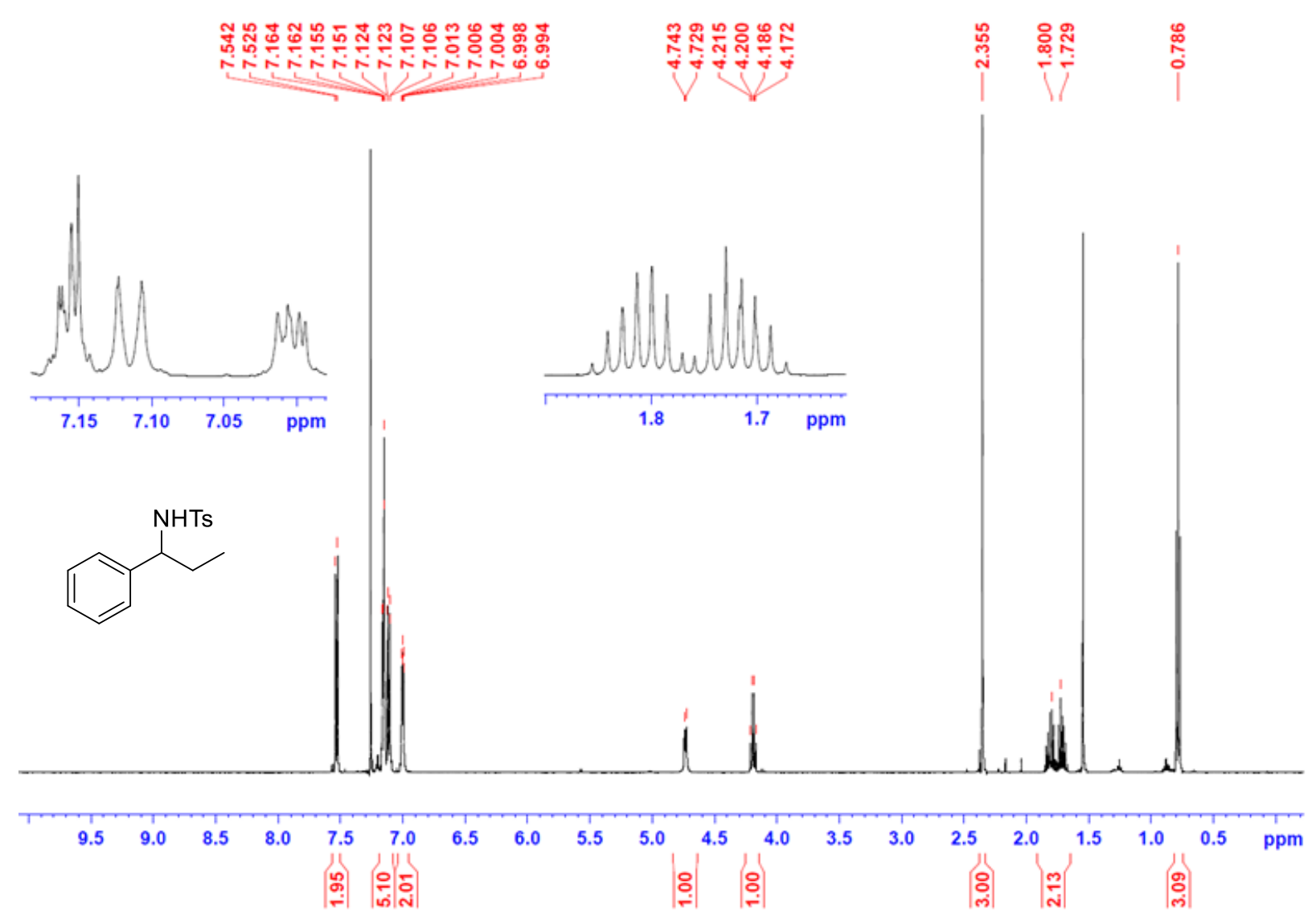

Figure S10. ${ }^{1} \mathrm{H}$ NMR of 4-Methyl-N-(1-phenylpropyl)benzenesulfonamide in $\mathrm{CDCl}_{3}$. 

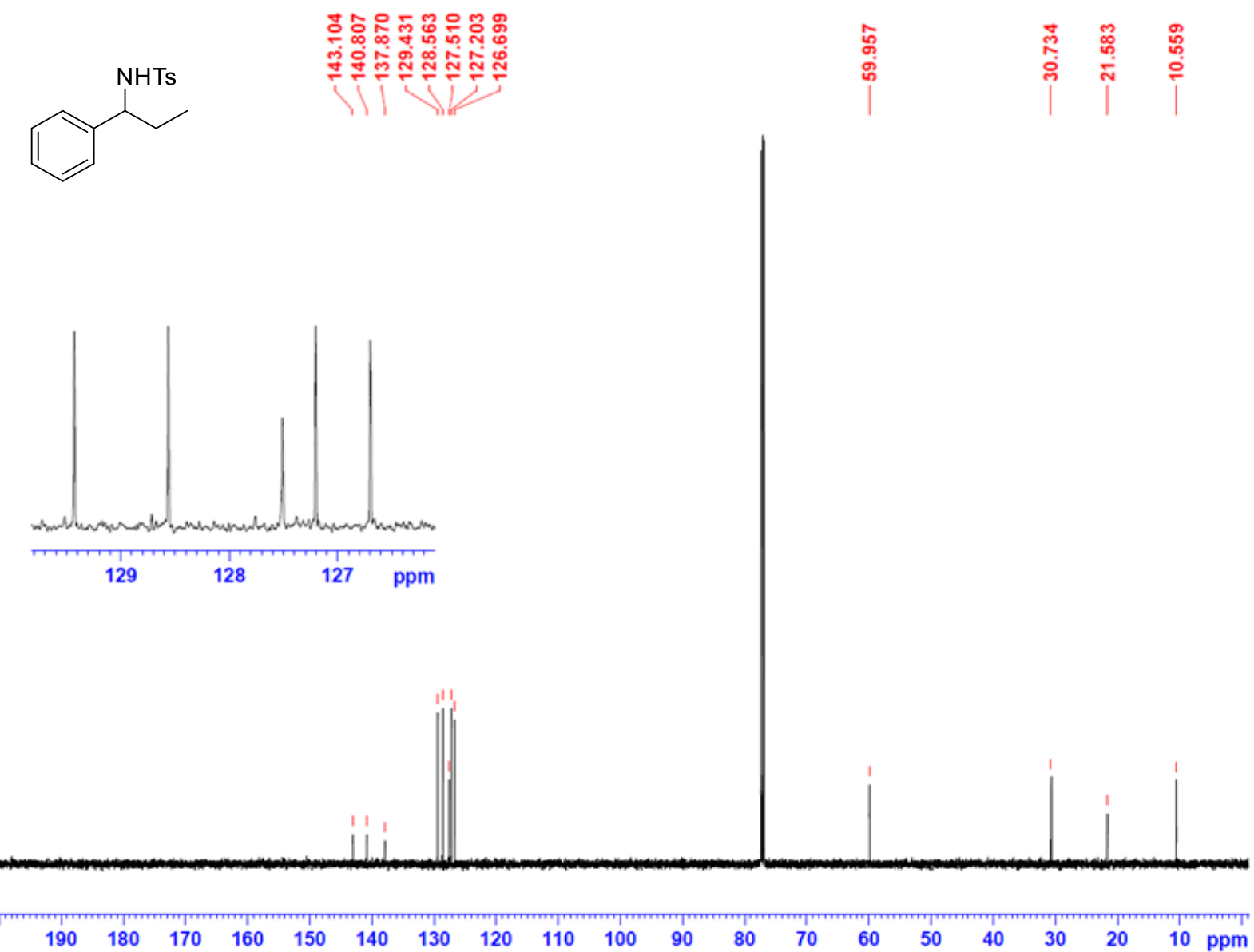

Figure S11. ${ }^{13} \mathrm{C}$ NMR of 4-Methyl-N-(1-phenylpropyl)benzenesulfonamide in $\mathrm{CDCl}_{3}$. 


\section{N-Benzhydryl-4-methylbenzenesulfonamide}<smiles>[NH3+]C(c1ccccc1)c1ccccc1</smiles>

In a glovebox, $\mathrm{Cu}\left(\mathrm{BF}_{4}\right)_{2} \cdot 6 \mathrm{H}_{2} \mathrm{O}(23.0 \mathrm{mg}, 63.0 \mu \mathrm{mol}, 10.0 \mathrm{~mol} \%)$, ligand $\mathbf{A}(30.0 \mathrm{mg}, 63 \mu \mathrm{mol}$, $10.0 \mathrm{~mol} \%$ ), $\mathrm{TsNH}_{2}$ (324 mg, $1.88 \mathrm{mmol}, 3.00$ equiv), $\mathrm{N}$-Fluorobenzenesulfonimide (593 mg, $1.88 \mathrm{mmol}, 3.00$ equiv) and diphenylmethane (104 $\mu \mathrm{L}, 105.1 \mathrm{mg}, 0.625 \mathrm{mmol}, 1.00$ equiv.) were added in this sequence to a $20 \mathrm{~mL}$ scintillation vial equipped with a Teflon-coated stir bar. The vial was sealed with a Teflon-lined vial cap and heated to $110^{\circ} \mathrm{C}$ on a pre-heated vial plate under vigorous stirring $(1500 \mathrm{rpm})$. After $48 \mathrm{~h}$, the vial was taken off the heating block and the mixture was allowed to cool to room temperature. The solvent was removed under reduced pressure. 15 $\mathrm{mL} \mathrm{CHCl}_{3}$ and $2 \mathrm{~g} \mathrm{CaCO}_{3}$ were added into the mixture and the resulting suspension was filtered through Celite. The filtrate was concentrated under vacuum (rotary evaporator) to yield the crude product which was purified by column chromatography using ethyl acetate/hexane (1:4) as eluent. $154 \mathrm{mg}$ (73\% yield) of pure title compound was obtained. The ${ }^{1} \mathrm{H}$ and ${ }^{13} \mathrm{C}$ NMR match the literature reported spectra. ${ }^{4}$

${ }^{1} \mathrm{H}$ NMR $\left(500 \mathrm{MHz}, \mathrm{CDCl}_{3}, 25^{\circ} \mathrm{C}\right): \delta[\mathrm{ppm}]=7.56(\mathrm{~d}, 2 \mathrm{H}), 7.24-7.17(\mathrm{~m}, 6 \mathrm{H}), 7.13(\mathrm{~d}$, 2H), $7.12-7.08(\mathrm{~s}, 4 \mathrm{H}), 5.57(\mathrm{~d}, 1 \mathrm{H}), 5.09(\mathrm{~d}, 1 \mathrm{H}), 2.39$ (s, 3H);

${ }^{13} \mathrm{C}\left(\mathrm{NMR} 125 \mathrm{MHz}, \mathrm{CDCl}_{3}, 25{ }^{\circ} \mathrm{C}\right): \delta[\mathrm{ppm}]=143.3(\mathrm{~s}), 140.7(\mathrm{~s}), 137.5(\mathrm{~s}), 129.5(\mathrm{~s})$, 128.7 (s), 127.7 (s), 127.5 (s), 127.4 (s), 61.5 (s), 21.5 (s); 


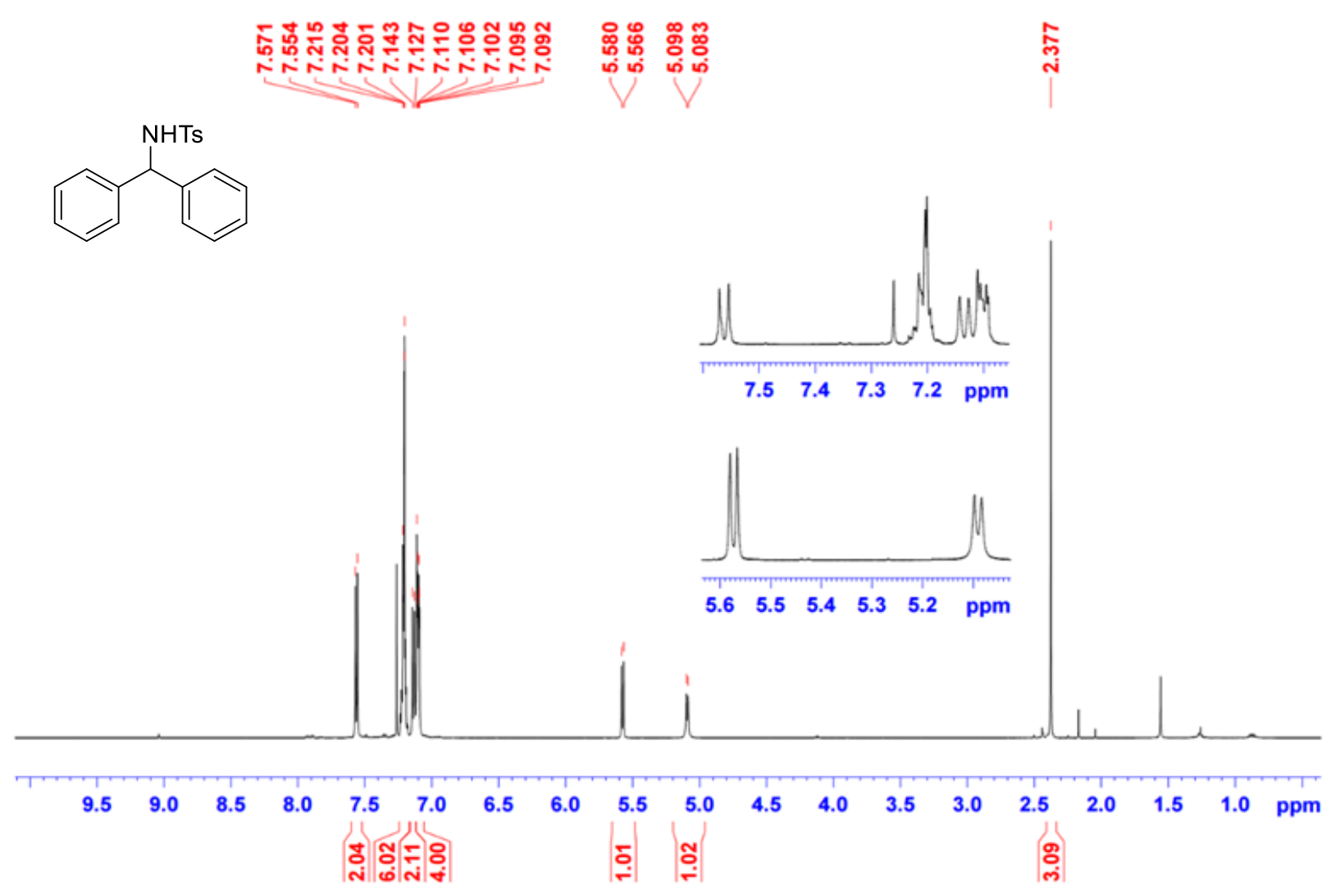

Figure S12. ${ }^{1} \mathrm{H}$ NMR of N-benzhydryl-4-methylbenzenesulfonamide in $\mathrm{CDCl}_{3}$. 


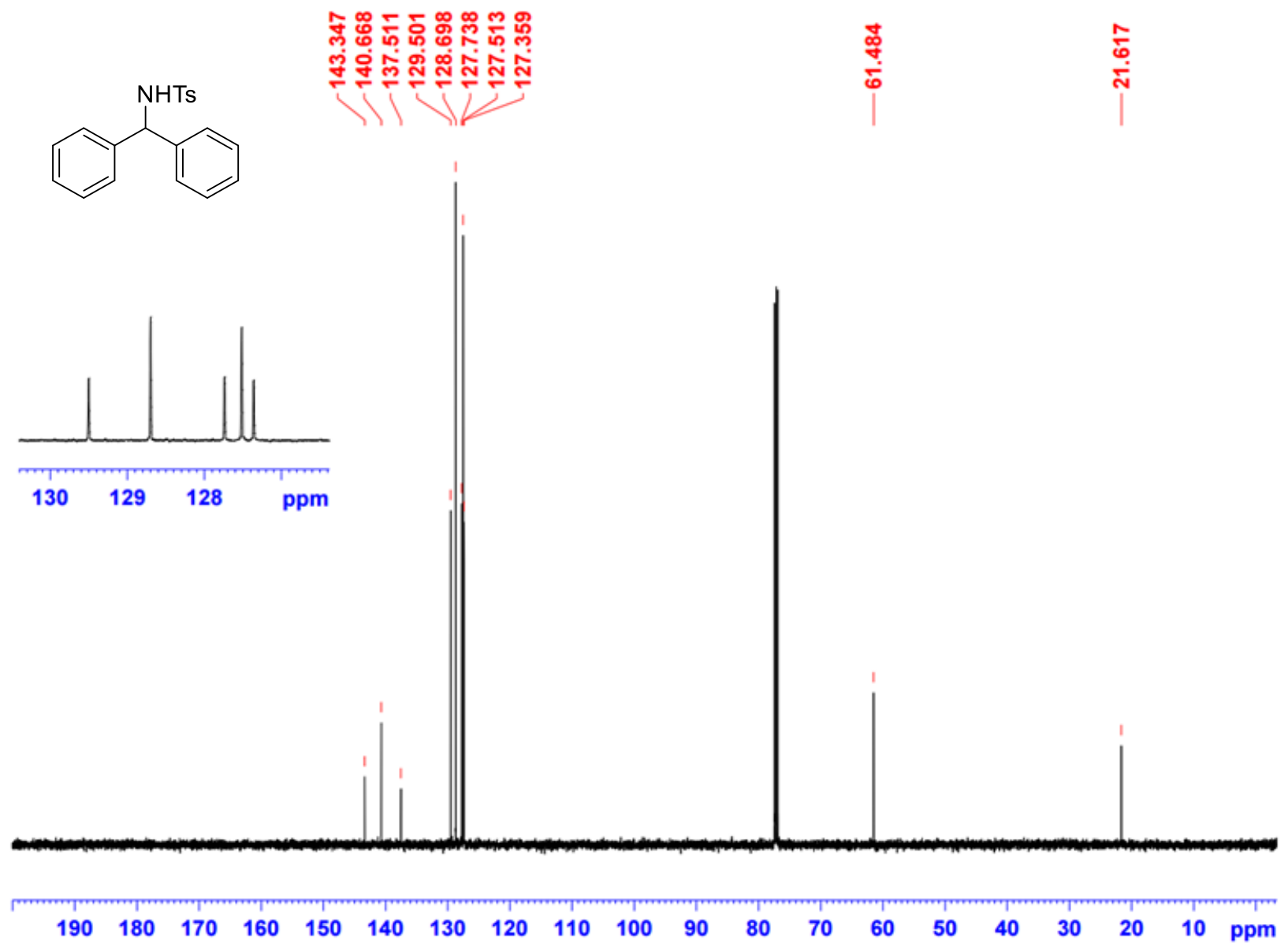

Figure S13. ${ }^{13} \mathrm{C}$ NMR of N-benzhydryl-4-methylbenzenesulfonamide in $\mathrm{CDCl}_{3}$. 


\section{N-(1-Phenylethyl)methanesulfonamide}<smiles>CNC(C)c1ccccc1</smiles>

In a glovebox, $\mathrm{Cu}\left(\mathrm{BF}_{4}\right)_{2} \cdot 6 \mathrm{H}_{2} \mathrm{O}(23.0 \mathrm{mg}, 63.0 \mu \mathrm{mol}, 10.0 \mathrm{~mol} \%)$, ligand $\mathbf{A}(30.0 \mathrm{mg}, 63 \mu \mathrm{mol}$, $10.0 \mathrm{~mol} \%$ ), $\mathrm{MsNH}_{2}$ (178 mg, $1.88 \mathrm{mmol}, 3.00$ equiv), $N$-Fluorobenzenesulfonimide (593 mg, $1.88 \mathrm{mmol}, 3.00$ equiv) and ethylbenzene $(80 \mu \mathrm{L}, 13.3 \mathrm{mg}, 0.625 \mathrm{mmol}, 1.00$ equiv. $)$ were added in this sequence to a $20 \mathrm{~mL}$ scintillation vial equipped with a Teflon-coated stir bar. The vial was sealed with a Teflon-lined vial cap and heated to $110{ }^{\circ} \mathrm{C}$ on a pre-heated vial plate under vigorous stirring $(1500 \mathrm{rpm})$. After $48 \mathrm{~h}$, the vial was taken off the heating block and the mixture was allowed to cool to room temperature. The solvent was removed under reduced pressure. 15 $\mathrm{mL} \mathrm{CHCl}_{3}$ and $2 \mathrm{~g} \mathrm{CaCO}_{3}$ was added into reaction mixture, followed by a filtration through a Celite plug. The filtrate was concentrated under vacuum (rotary evaporator) to yield the crude product which was purified by column chromatography using ethyl acetate/ hexane (1:4) as eluent. $105 \mathrm{mg}$ (83\% yield) of pure title compound was obtained. The ${ }^{1} \mathrm{H}$ and ${ }^{13} \mathrm{C} \mathrm{NMR}$ match the literature reported spectra. ${ }^{5}$

${ }^{1} \mathrm{H}$ NMR $\left(500 \mathrm{MHz}, \mathrm{CDCl}_{3}, 25^{\circ} \mathrm{C}\right): \delta[\mathrm{ppm}]=7.42-7.26(\mathrm{~m}, 5 \mathrm{H}), 5.68(\mathrm{~d}, 1 \mathrm{H}) ; 4.65(\mathrm{p}$, $1 \mathrm{H}), 2.62(\mathrm{~s}, 3 \mathrm{H}), 1.54(\mathrm{~d}, 3 \mathrm{H})$;

${ }^{13} \mathrm{C}\left(\mathrm{NMR} 125 \mathrm{MHz}, \mathrm{CDCl}_{3}, 25^{\circ} \mathrm{C}\right): \delta[\mathrm{ppm}]=142.7(\mathrm{~s}), 128.8(\mathrm{~s}), 127.7(\mathrm{~s}), 126.2(\mathrm{~s})$, $53.7(\mathrm{~s}), 41.5(\mathrm{~s}), 23.9(\mathrm{~s})$; 


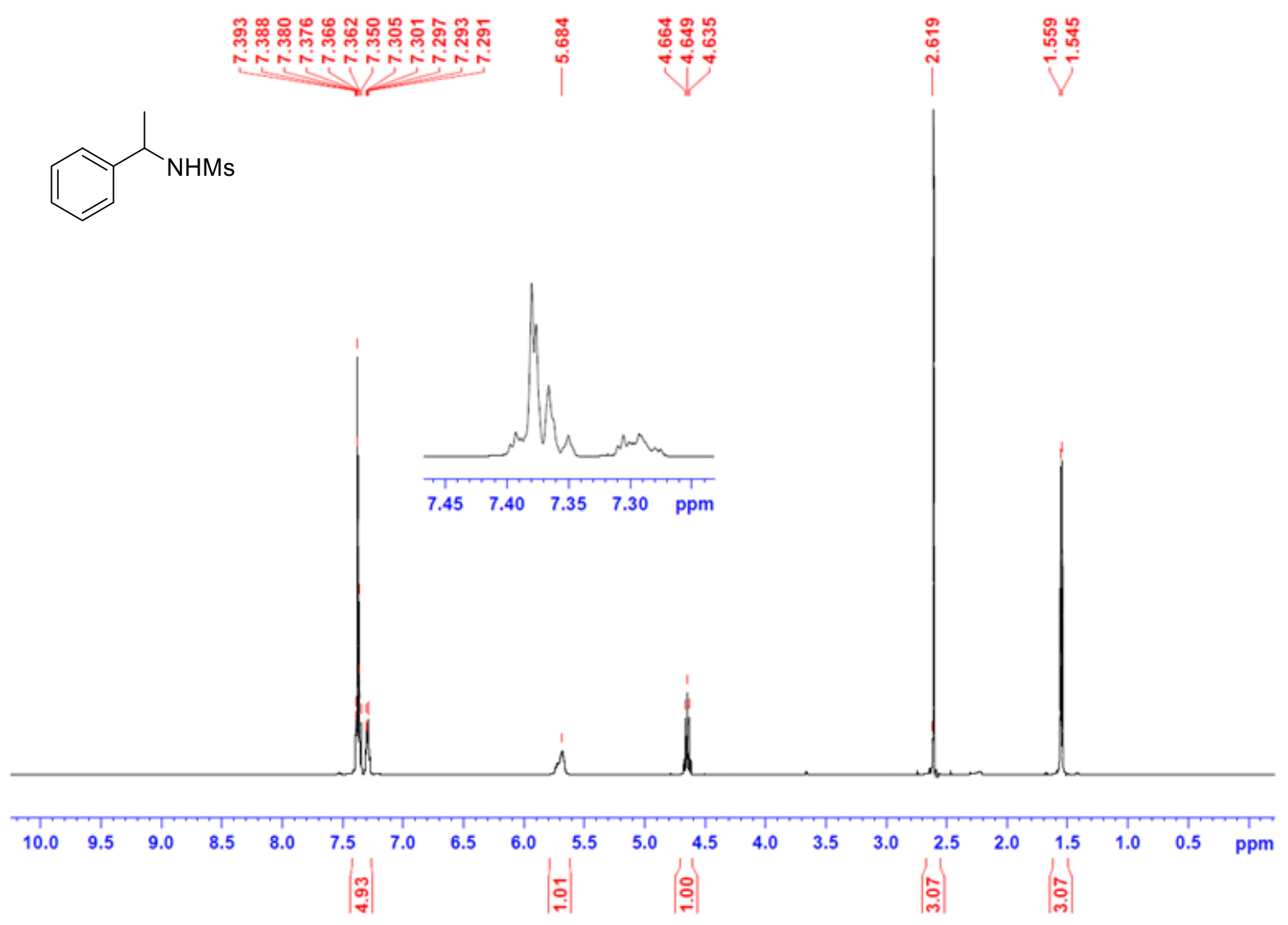

Figure S14. ${ }^{1} \mathrm{H}$ NMR of $\mathrm{N}$-(1-Phenylethyl)methanesulfonamide in $\mathrm{CDCl}_{3}$. 


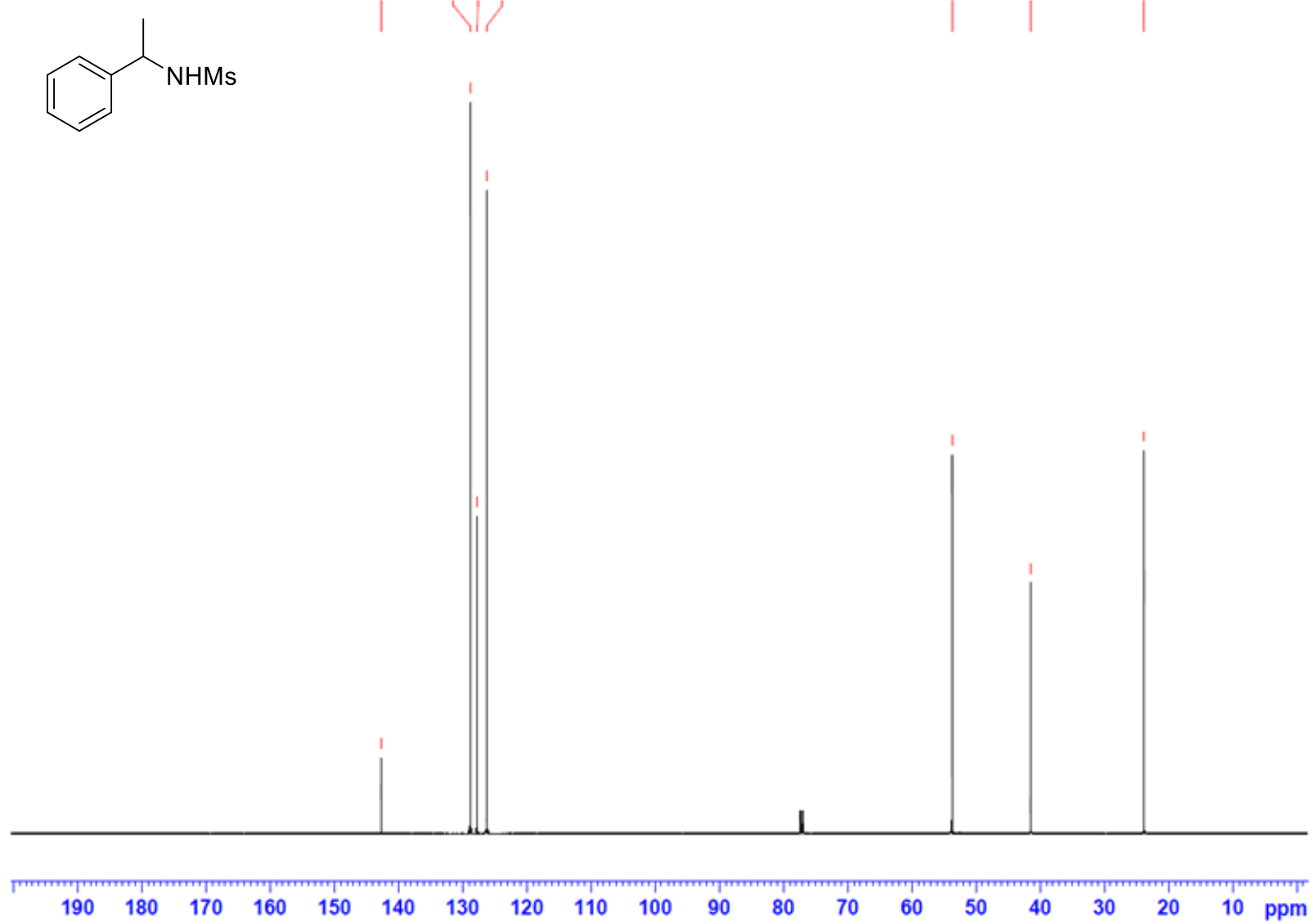

Figure S15. ${ }^{13} \mathrm{C}$ NMR of N-(1-Phenylethyl)methanesulfonamide in $\mathrm{CDCl}_{3}$. 


\section{N-(1-phenylethyl)benzenesulfonamide}<smiles>CC(NS(=O)(=O)c1ccccc1)c1ccccc1</smiles>

In a glovebox, $\mathrm{Cu}\left(\mathrm{BF}_{4}\right)_{2} \cdot 6 \mathrm{H}_{2} \mathrm{O}(23.0 \mathrm{mg}, 63.0 \mu \mathrm{mol}, 10.0 \mathrm{~mol} \%)$, ligand $\mathbf{A}(30.0 \mathrm{mg}, 63 \mu \mathrm{mol}$, $10.0 \mathrm{~mol} \%)$, Benzenesulfonamide $(296 \mathrm{mg}, \quad 1.88 \mathrm{mmol}, 3.00$ equiv), $N$ Fluorobenzenesulfonimide (593 mg, $1.88 \mathrm{mmol}, 3.00$ equiv) and ethylbenzene ( $80 \mu \mathrm{L}, 13.3 \mathrm{mg}$, $0.625 \mathrm{mmol}, 1.00$ equiv.) were added in this sequence to a $20 \mathrm{~mL}$ scintillation vial equipped with a Teflon-coated stir bar. The vial was sealed with a Teflon-lined vial cap and heated to $110^{\circ} \mathrm{C}$ on a pre-heated vial plate under vigorous stirring (1500 rpm). After $48 \mathrm{~h}$, the vial was taken off the heating block and the mixture was allowed to cool to room temperature. The solvent was removed under reduced pressure. $15 \mathrm{~mL} \mathrm{CHCl}_{3}$ and $2 \mathrm{~g} \mathrm{CaCO}_{3}$ were added into the mixture and the resulting suspension was filtered through Celite. The filtrate was concentrated under vacuum (rotary evaporator) to yield the crude product which was purified by column chromatography using ethyl acetate/hexane (1:4) as eluent. $141 \mathrm{mg}$ (85\% yield) of pure title compound was obtained. The ${ }^{1} \mathrm{H}$ and ${ }^{13} \mathrm{C}$ NMR match the literature reported spectra. ${ }^{5}$

${ }^{1} \mathrm{H}$ NMR $\left(500 \mathrm{MHz}, \mathrm{CDCl}_{3}, 25^{\circ} \mathrm{C}\right): \delta[\mathrm{ppm}]=7.75-7.71(\mathrm{~m}, 2 \mathrm{H}), 7.47(\mathrm{td}, \mathrm{J}=6.4,1 \mathrm{H})$, 7.39-7.32 (m, 2H), 7.20-7.06 (m, 5H), $5.44(\mathrm{~d}, 1 \mathrm{H}), 4.50(\mathrm{p}, 1 \mathrm{H}), 1.42(\mathrm{~d}, 3 \mathrm{H})$;

${ }^{13} \mathrm{C}\left(\mathrm{NMR} 125 \mathrm{MHz}, \mathrm{CDCl}_{3}, 25^{\circ} \mathrm{C}\right): \delta[\mathrm{ppm}]=142.0(\mathrm{~s}), 140.8(\mathrm{~s}), 132.4(\mathrm{~s}), 128.9(\mathrm{~s})$, 128.6 (s), 127.5 (s), 127.1 (s), 126.2 (s), 53.8 (s), 23.7 (s); 


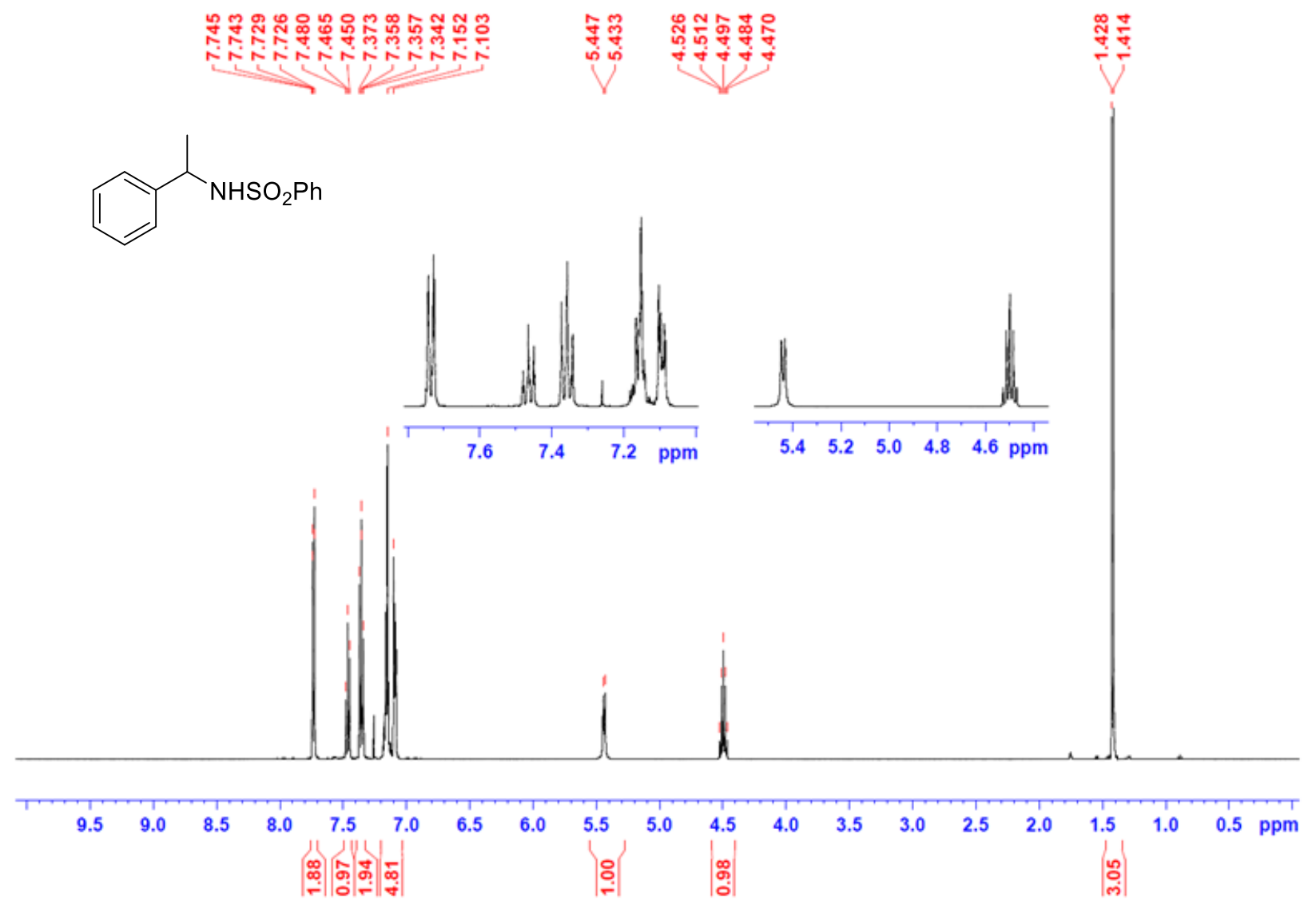

Figure S16. ${ }^{1} \mathrm{H}$ NMR of N-(1-phenylethyl)benzenesulfonamide in $\mathrm{CDCl}_{3}$. 


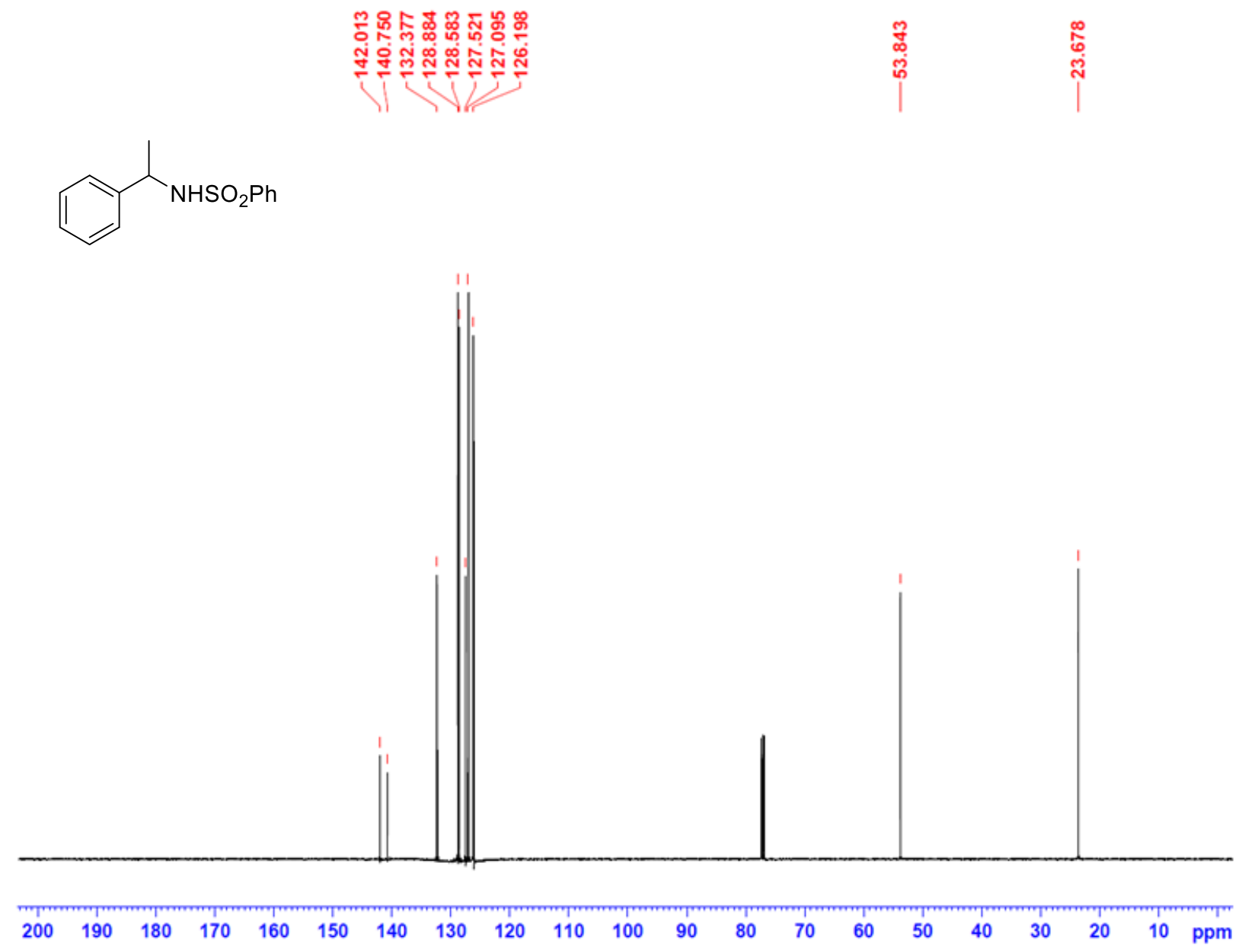

Figure S17. ${ }^{13} \mathrm{C}$ NMR of $\mathrm{N}$-(1-phenylethyl)benzenesulfonamide in $\mathrm{CDCl}_{3}$. 


\section{4-Methyl-N-(1-(naphthalen-2-yl)ethyl)benzenesulfonamide}

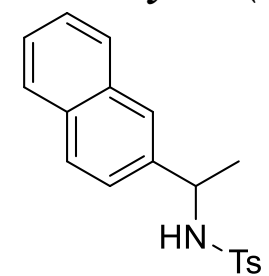

In a glovebox, $\mathrm{Cu}\left(\mathrm{BF}_{4}\right)_{2} \cdot 6 \mathrm{H}_{2} \mathrm{O}(23.0 \mathrm{mg}, 63.0 \mu \mathrm{mol}, 10.0 \mathrm{~mol} \%)$, ligand $\mathbf{A}(30.0 \mathrm{mg}, 63 \mu \mathrm{mol}$, $10.0 \mathrm{~mol} \%$ ), $\mathrm{TsNH}_{2}$ (324 mg, $1.88 \mathrm{mmol}, 3.00$ equiv), $N$-Fluorobenzenesulfonimide $(593 \mathrm{mg}$, $1.88 \mathrm{mmol}, 3.00$ equiv) and 2-ethylnaphthalene $(99 \mu \mathrm{L}, 97.6 \mathrm{mg}, 0.625 \mathrm{mmol}, 1.00$ equiv.) were added in this sequence to a $20 \mathrm{~mL}$ scintillation vial equipped with a Teflon-coated stir bar. The vial was sealed with a Teflon-lined vial cap and heated to $110{ }^{\circ} \mathrm{C}$ on a pre-heated vial plate under vigorous stirring (1500 rpm). After $48 \mathrm{~h}$, the vial was taken off the heating block and the mixture was allowed to cool to room temperature. The solvent was removed under reduced pressure. 15 $\mathrm{mL} \mathrm{CHCl}_{3}$ and $2 \mathrm{~g} \mathrm{CaCO}_{3}$ were added into the mixture and the resulting suspension was filtered through Celite. The filtrate was concentrated under vacuum (rotary evaporator) to yield the crude product which was purified by column chromatography using ethyl acetate/ hexane (1:4) as eluent. $30 \mathrm{mg}$ (15\% yield) of pure title compound was obtained. The ${ }^{1} \mathrm{H}$ and ${ }^{13} \mathrm{C}$ NMR match the literature reported spectra. ${ }^{6}$

${ }^{1} \mathrm{H} \mathrm{NMR}\left(500 \mathrm{MHz}, \mathrm{CDCl}_{3}, 25^{\circ} \mathrm{C}\right): \delta[\mathrm{ppm}]=7.74(\mathrm{t}, 1 \mathrm{H}), 7.65(\mathrm{~d}, 2 \mathrm{H}), 7.58(\mathrm{~d}, 2 \mathrm{H})$, 7.40-7.49 (m, 3H), $7.22(\mathrm{~d}, 1 \mathrm{H}), 7.00(\mathrm{~d}, 2 \mathrm{H}), 5.43(\mathrm{~d}, 1 \mathrm{H}), 4.64(\mathrm{dt}, 1 \mathrm{H}), 2.22(\mathrm{~s}, 3 \mathrm{H}), 1.50(\mathrm{~d}$, $3 \mathrm{H})$;

${ }^{13} \mathrm{C}\left(\mathrm{NMR} 125 \mathrm{MHz}, \mathrm{CDCl}_{3}, 25{ }^{\circ} \mathrm{C}\right): \delta[\mathrm{ppm}]=143.1(\mathrm{~s}), 139.2(\mathrm{~s}), 137.7(\mathrm{~s}), 133.2(\mathrm{~s})$, $132.8(\mathrm{~s}), 129.4(\mathrm{~s}), 128.5(\mathrm{~s}), 127.9(\mathrm{~s}), 127.6(\mathrm{~s}), 127.2(\mathrm{~s}), 126.2(\mathrm{~s}), 126.0(\mathrm{~s}), 125.2(\mathrm{~s}), 124.2$ (s), $54.0(\mathrm{~s}), 23.5(\mathrm{~s}), 21.3(\mathrm{~s})$; 


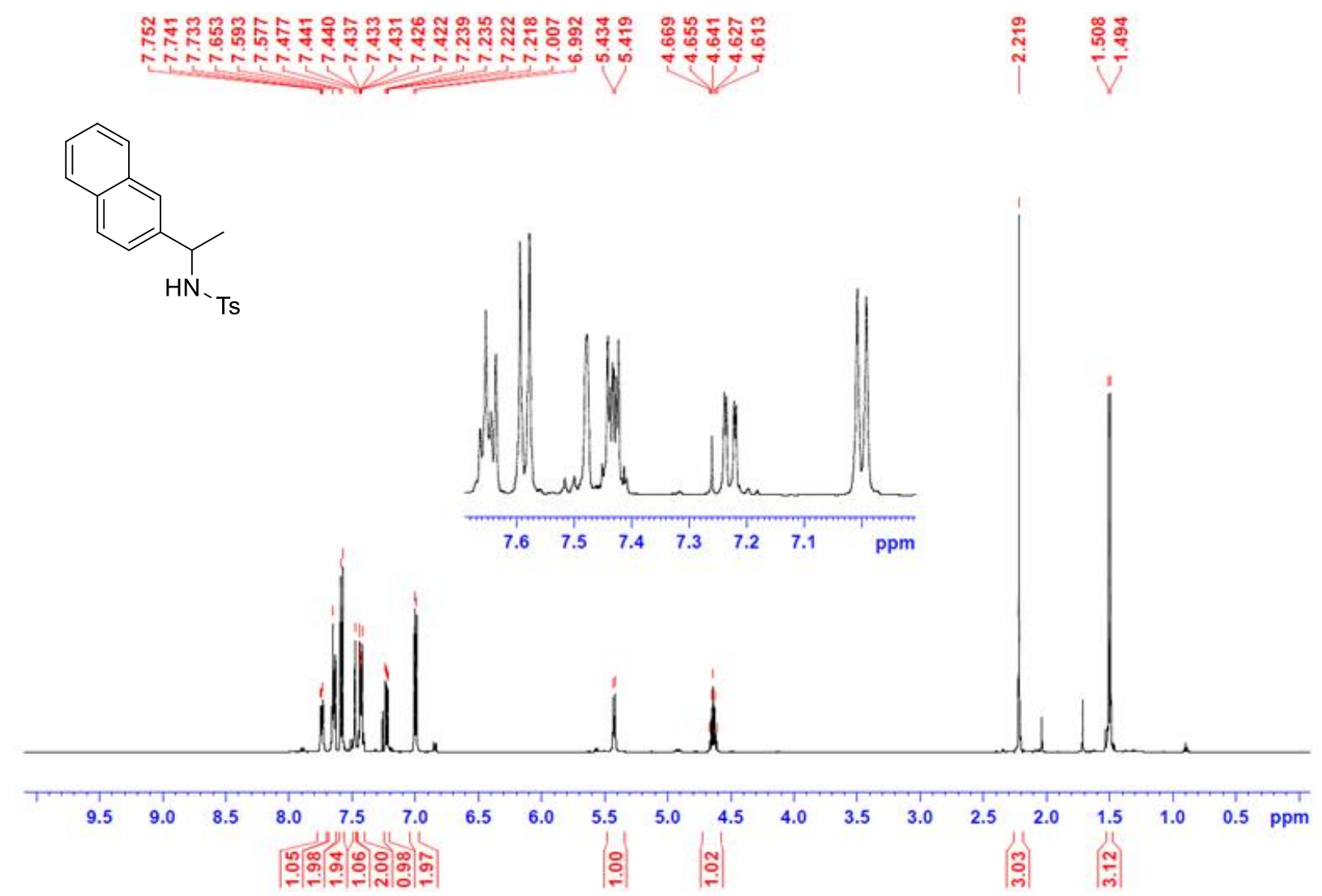

Figure S18. ${ }^{1} \mathrm{H}$ NMR of 4-Methyl-N-(1-(naphthalen-2-yl)ethyl)benzenesulfonamide in $\mathrm{CDCl}_{3}$. 


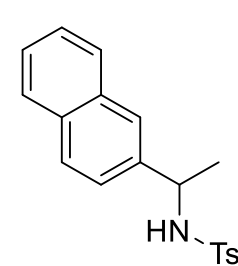

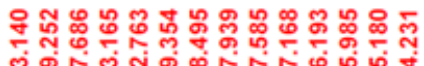

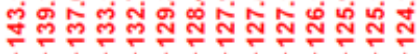
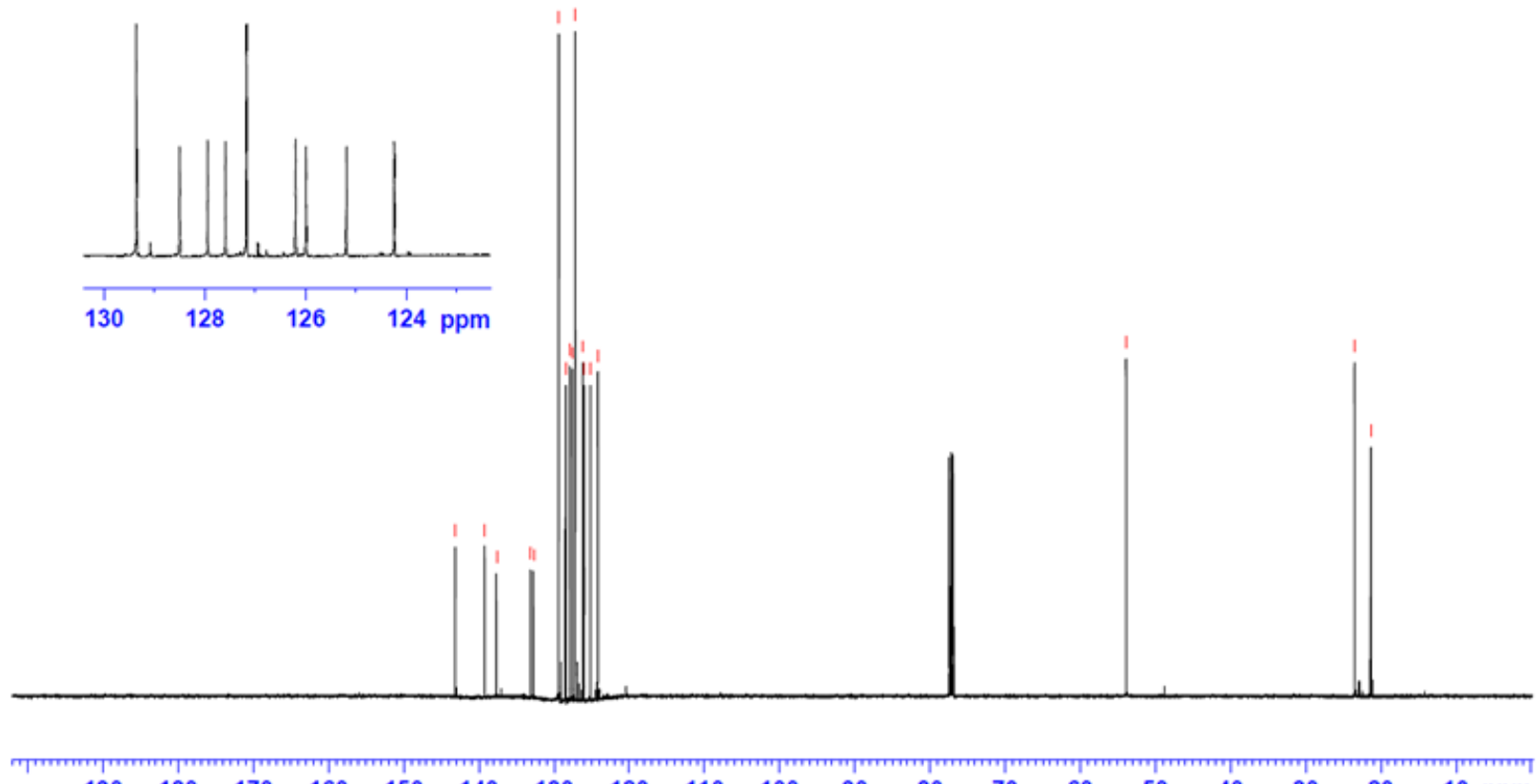

$\begin{array}{llllllllllllllllllll}190 & 180 & 170 & 160 & 150 & 140 & 130 & 120 & 110 & 100 & 90 & 80 & 70 & 60 & 50 & 40 & 30 & 20 & 10 & \mathrm{ppm}\end{array}$

Figure S19. ${ }^{13} \mathrm{C}$ NMR of 4-Methyl-N-(1-(naphthalen-2-yl)ethyl)benzenesulfonamide in $\mathrm{CDCl}_{3}$. 


\section{N,4-Dimethyl-N-(1-phenylethyl)benzenesulfonamide}<smiles>CC(c1ccccc1)[N+](C)(C)C</smiles>

In a glovebox, $\mathrm{Cu}\left(\mathrm{BF}_{4}\right)_{2} \cdot 6 \mathrm{H}_{2} \mathrm{O}(23.0 \mathrm{mg}, 63.0 \mu \mathrm{mol}, 10.0 \mathrm{~mol} \%)$, ligand $\mathbf{A}(30.0 \mathrm{mg}, 63 \mu \mathrm{mol}$, $10.0 \mathrm{~mol} \%)$, N,4-dimethylbenzenesulfonamide (347 mg, $1.88 \mathrm{mmol}, 3.00$ equiv), $N$ Fluorobenzenesulfonimide (593 mg, $1.88 \mathrm{mmol}, 3.00$ equiv) and ethylbenzene ( $80 \mu \mathrm{L}, 13.3 \mathrm{mg}$, $0.625 \mathrm{mmol}, 1.00$ equiv.) were added in this sequence to a $20 \mathrm{~mL}$ scintillation vial equipped with a Teflon-coated stir bar. The vial was sealed with a Teflon-lined vial cap and heated to $110{ }^{\circ} \mathrm{C}$ on a pre-heated vial plate under vigorous stirring $(1500 \mathrm{rpm})$. After $48 \mathrm{~h}$, the vial was taken off the heating block and the mixture was allowed to cool to room temperature. The solvent was removed under reduced pressure. $15 \mathrm{~mL} \mathrm{CHCl}_{3}$ and $2 \mathrm{~g} \mathrm{CaCO}_{3}$ were added into the mixture and the resulting suspension was filtered through Celite. The filtrate was concentrated under vacuum (rotary evaporator) to yield the crude product which was purified by column chromatography using ethyl acetate/ hexane (1:4) as eluent. $52 \mathrm{mg}$ (29\% yield) of pure title compound was obtained. The ${ }^{1} \mathrm{H}$ and ${ }^{13} \mathrm{C}$ NMR match the literature reported spectra. ${ }^{5}$

${ }^{1} \mathrm{H}$ NMR $\left(500 \mathrm{MHz}, \mathrm{CDCl}_{3}, 25^{\circ} \mathrm{C}\right): \delta[\mathrm{ppm}]=7.74(\mathrm{dt}, 2 \mathrm{H}), 7.33-7.21(\mathrm{~m}, 7 \mathrm{H}), 5.29(\mathrm{q}$, 1H), 2.57 (s, 3H), 2.44 (s, 3H), $1.29(\mathrm{~d}, 3 \mathrm{H})$;

${ }^{13} \mathrm{C}\left(\mathrm{NMR} 125 \mathrm{MHz}, \mathrm{CDCl}_{3}, 25{ }^{\circ} \mathrm{C}\right): \delta[\mathrm{ppm}]=143.2(\mathrm{~s}), 140.0(\mathrm{~s}), 137.4(\mathrm{~s}), 129.8(\mathrm{~s})$, 128.5 (s), 127.6 (s), $127.4(\mathrm{~s}), 127.2$ (s), 54.8 (s), 28.5 (s), 21.6 (s), $15.3(\mathrm{~s})$; 


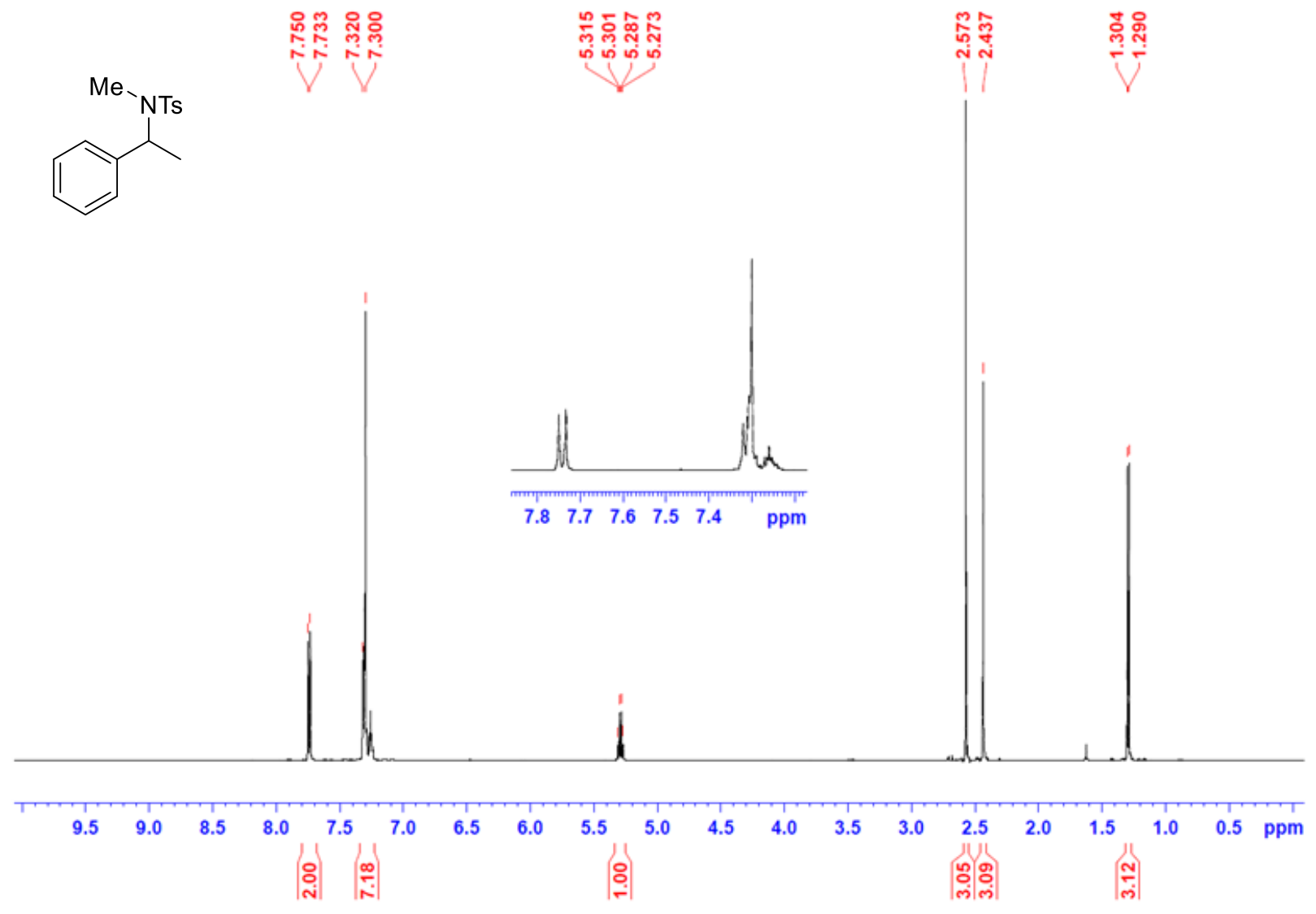

Figure S20. ${ }^{1} \mathrm{H}$ NMR of N,4-Dimethyl-N-(1-phenylethyl)benzenesulfonamide in $\mathrm{CDCl}_{3}$. 


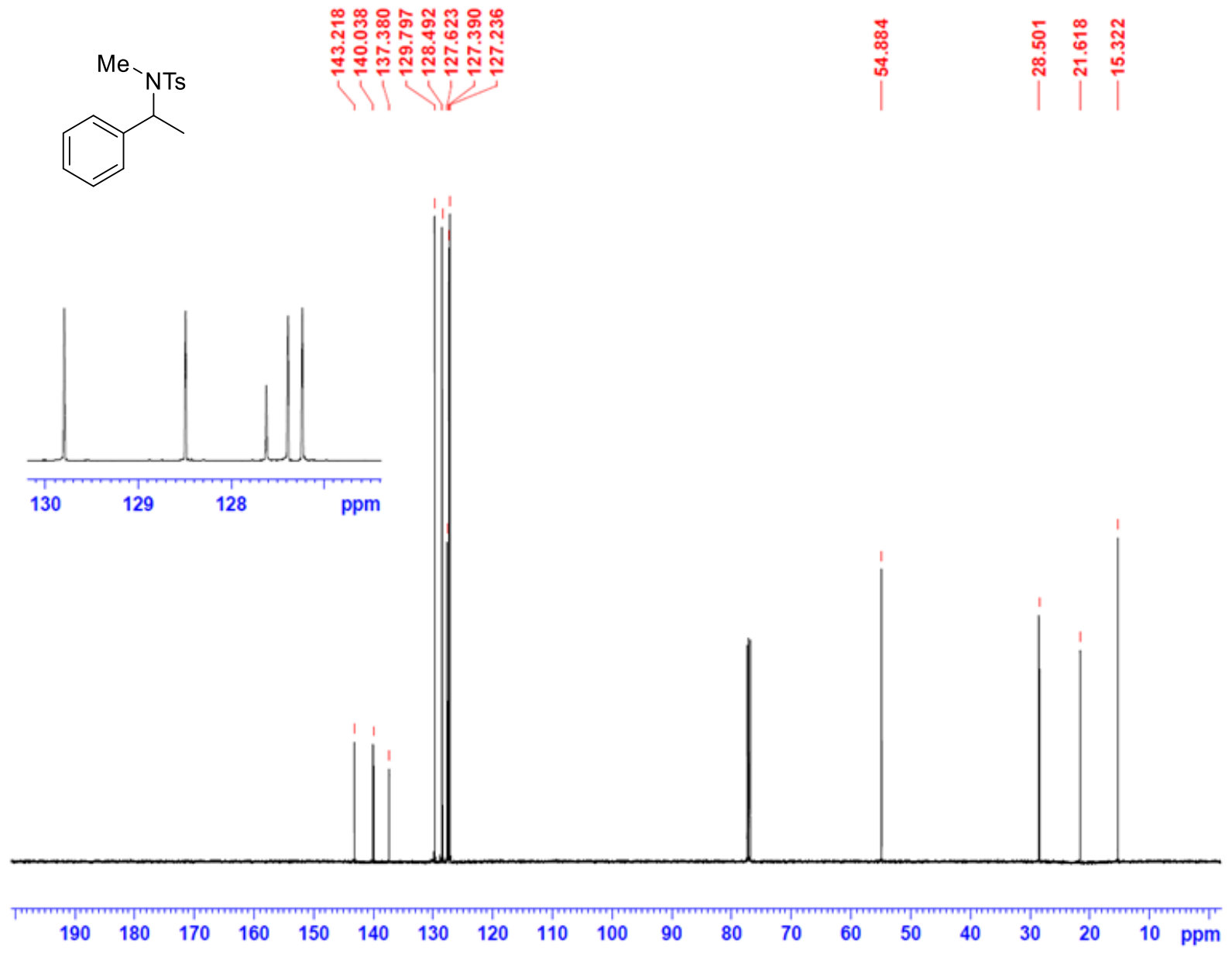

Figure S21. ${ }^{13} \mathrm{C}$ NMR of N,4-Dimethyl-N-(1-phenylethyl)benzenesulfonamide in $\mathrm{CDCl}_{3}$. 


\section{N-(1-Phenylethyl)naphthalene-2-sulfonamide}<smiles>CC(NS(=O)(=O)c1ccc2ccccc2c1)c1ccccc1</smiles>

In a glovebox, $\mathrm{Cu}\left(\mathrm{BF}_{4}\right)_{2} \cdot 6 \mathrm{H}_{2} \mathrm{O}(23.0 \mathrm{mg}, 63.0 \mu \mathrm{mol}, 10.0 \mathrm{~mol} \%)$, ligand $\mathbf{A}(30.0 \mathrm{mg}, 63 \mu \mathrm{mol}$, $10.0 \mathrm{~mol} \%), \mathrm{N}-(1-P h e n y l e t h y l) n a p h t h a l e n e-2-s u l f o n a m i d e ~(389 \mathrm{mg}, 1.88 \mathrm{mmol}, 3.00$ equiv), $N$ Fluorobenzenesulfonimide (593 mg, $1.88 \mathrm{mmol}, 3.00$ equiv) and ethylbenzene ( $80 \mu \mathrm{L}, 13.3 \mathrm{mg}$, $0.625 \mathrm{mmol}, 1.00$ equiv.) were added in this sequence to a $20 \mathrm{~mL}$ scintillation vial equipped with a Teflon-coated stir bar. The vial was sealed with a Teflon-lined vial cap and heated to $110{ }^{\circ} \mathrm{C}$ on a pre-heated vial plate under vigorous stirring $(1500 \mathrm{rpm})$. After $48 \mathrm{~h}$, the vial was taken off the heating block and the mixture was allowed to cool to room temperature. The solvent was removed under reduced pressure. $15 \mathrm{~mL} \mathrm{CHCl}_{3}$ and $2 \mathrm{~g} \mathrm{CaCO}_{3}$ were added into the mixture and the resulting suspension was filtered through Celite. The filtrate was concentrated under vacuum (rotary evaporator) to yield the crude product which was purified by column chromatography using ethyl acetate/ hexane (1:4) as eluent. $23 \mathrm{mg}$ (12\% yield) of pure title compound was obtained as white solid.

${ }^{1} \mathrm{H}$ NMR $\left(500 \mathrm{MHz}, \mathrm{CDCl}_{3}, 25^{\circ} \mathrm{C}\right): \delta[\mathrm{ppm}]=8.26(\mathrm{~d}, 1 \mathrm{H}), 7.89-7.82(\mathrm{~m}, 3 \mathrm{H}), 7.69(\mathrm{dd}$, $1 \mathrm{H}), 7.65-7.55(\mathrm{~m}, 2 \mathrm{H}), 7.14-7.04(\mathrm{~m}, 5 \mathrm{H}), 4.74(\mathrm{~d}, 1 \mathrm{H}), 4.54(\mathrm{q}, 1 \mathrm{H}), 4.64(\mathrm{dt}, 1 \mathrm{H}) 1.45(\mathrm{~d}, 3 \mathrm{H})$;

${ }^{13} \mathrm{C}\left(\mathrm{NMR} 125 \mathrm{MHz}, \mathrm{CDCl}_{3}, 25^{\circ} \mathrm{C}\right): \delta[\mathrm{ppm}]=141.8(\mathrm{~s}), 137.5(\mathrm{~s}), 134.8(\mathrm{~s}), 132.2(\mathrm{~s})$, 129.4 (s), 128.8 (s), 128.7 (s), 128.6 (s), 128.0 (s), 127.7 (s), 127.5 (s), 126.2 (s), 122.4 (s),53.9 (s), $23.7(\mathrm{~s})$;

IR (ATR): ${ }^{\tilde{v}}\left[\mathrm{~cm}^{-1}\right]=3274(\mathrm{~s}), 3059$ (s), 3031 (s), 2977 (s), 2932 (s), 2873 (s), 1658 (s), 1592 (s), 1539 (s), 1494 (s), 1454 (s), 1427 (s), 1379 (s), 1321 (s), 1272 (s), 1245 (s), 1207 (s), 1155 (s), 1131 (s), 1120 (s), 1075 (s), 1019 (s), 966 (s), 907 (s), 865 (s), 816 (s), 762 (s), 749 (s), $699(\mathrm{~s}), 662(\mathrm{~s}), 643(\mathrm{~s}), 619(\mathrm{~s})$;

HRMS: calcd. for $\mathrm{C}_{18} \mathrm{H}_{18} \mathrm{NO}_{2} \mathrm{~S},[\mathrm{M}+\mathrm{H}]^{+}$: 312.1058, found: 312.1042; 
<smiles>CC(NS(=O)(=O)c1ccc2ccccc2c1)c1ccccc1</smiles>
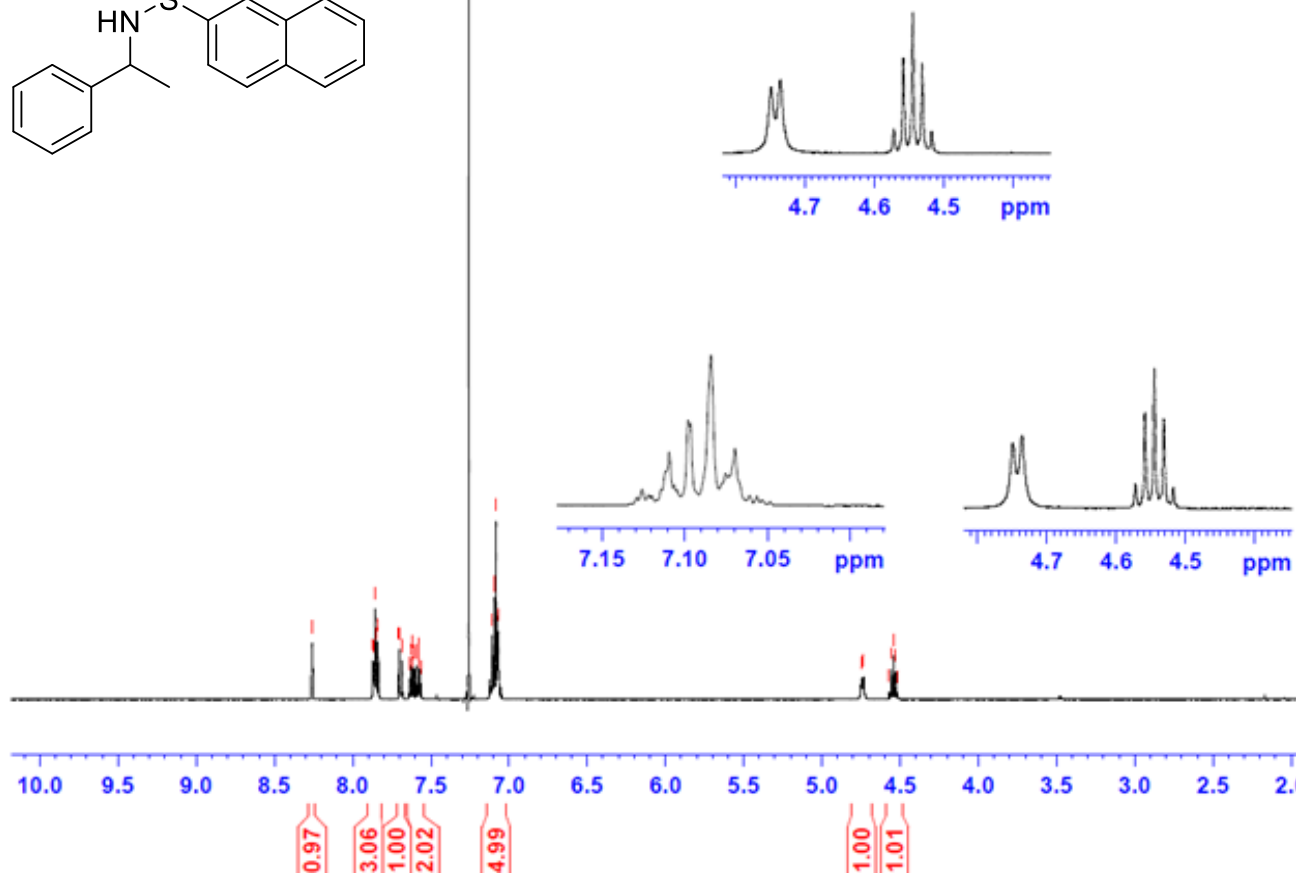

$3.5 \quad 3.0$

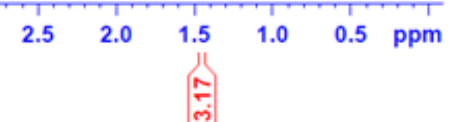

Figure S22. ${ }^{1} \mathrm{H}$ NMR of N-(1-Phenylethyl)naphthalene-2-sulfonamide in $\mathrm{CDCl}_{3}$. 


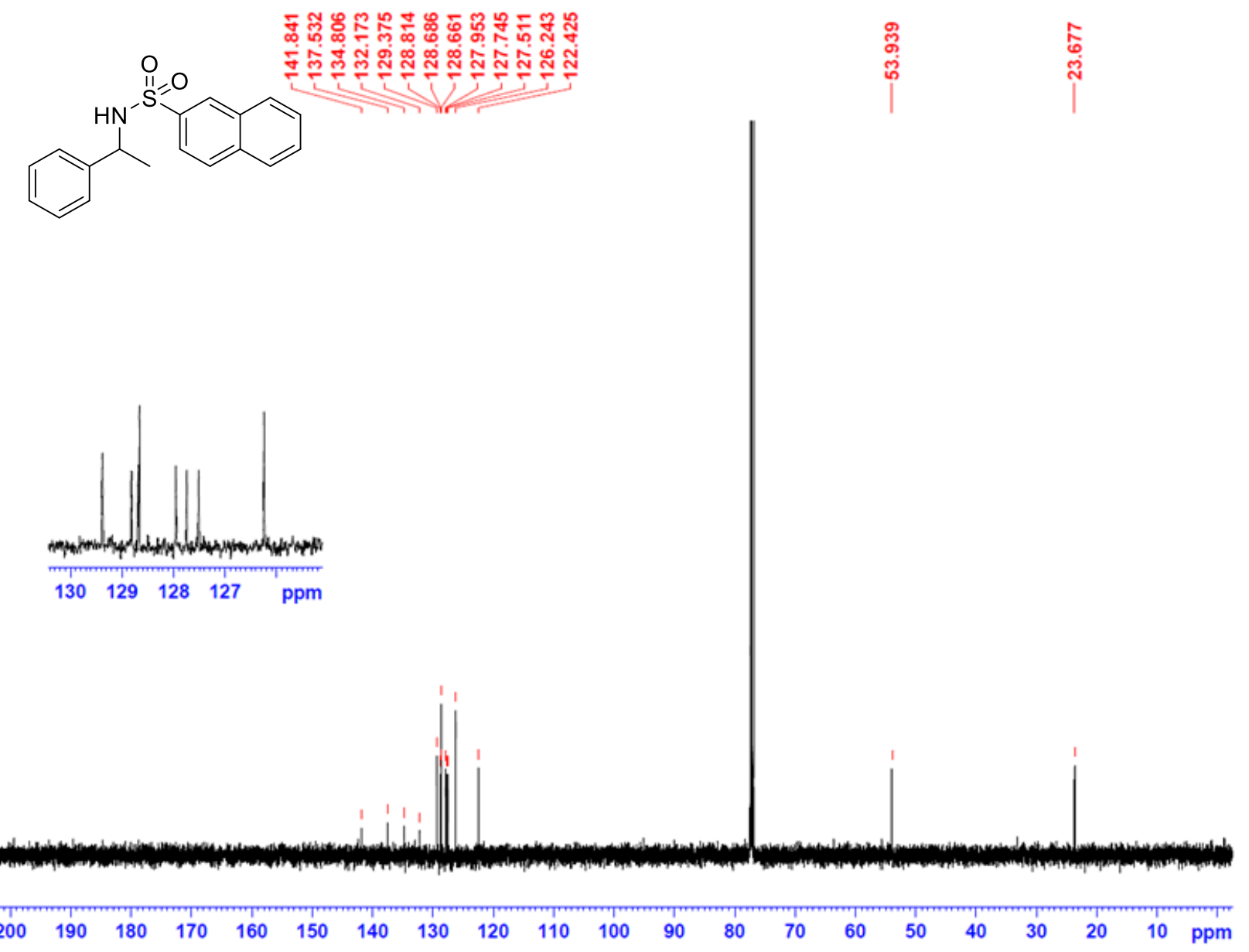

Figure S23. ${ }^{13} \mathrm{C}$ NMR of N-(1-Phenylethyl)naphthalene-2-sulfonamide in $\mathrm{CDCl}_{3}$. 


\section{N-(1-Phenylethyl)benzamide}<smiles>CC(NC(=O)c1ccccc1)c1ccccc1</smiles>

In a glovebox, $\mathrm{Cu}\left(\mathrm{BF}_{4}\right)_{2} \cdot 6 \mathrm{H}_{2} \mathrm{O}(23.0 \mathrm{mg}, 63.0 \mu \mathrm{mol}, 10.0 \mathrm{~mol} \%)$, ligand $\mathbf{A}(30.0 \mathrm{mg}, 63 \mu \mathrm{mol}$, $10.0 \mathrm{~mol} \%$ ), benzamide ( $227 \mathrm{mg}, 1.88 \mathrm{mmol}, 3.00$ equiv), $N$-Fluorobenzenesulfonimide (593 $\mathrm{mg}, 1.88 \mathrm{mmol}, 3.00$ equiv) and ethylbenzene ( $80 \mu \mathrm{L}, 13.3 \mathrm{mg}, 0.625 \mathrm{mmol}, 1.00$ equiv.) were added in this sequence to a $20 \mathrm{~mL}$ scintillation vial equipped with a Teflon-coated stir bar. The vial was sealed with a Teflon-lined vial cap and heated to $110^{\circ} \mathrm{C}$ on a pre-heated vial plate under vigorous stirring $(1500 \mathrm{rpm})$. After $48 \mathrm{~h}$, the vial was taken off the heating block and the mixture was allowed to cool to room temperature. The solvent was removed under reduced pressure. $15 \mathrm{~mL} \mathrm{CHCl}_{3}$ and $2 \mathrm{~g} \mathrm{CaCO}_{3}$ were added into the mixture and the resulting suspension was filtered through Celite. The filtrate was concentrated under vacuum (rotary evaporator) to yield the crude product which was purified by column chromatography using ethyl acetate/ petroleum ether (1:9 to $1: 1)$ as eluent. $31 \mathrm{mg}$ (22\% yield) of pure title compound was obtained. The ${ }^{1} \mathrm{H}$ and ${ }^{13} \mathrm{C}$ NMR match the literature reported spectra. ${ }^{7}$

${ }^{1} \mathrm{H}$ NMR $\left(500 \mathrm{MHz}, \mathrm{CDCl}_{3}, 25^{\circ} \mathrm{C}\right): \delta[\mathrm{ppm}]=7.77(\mathrm{~d}, 2 \mathrm{H}), 7.53-7.19(\mathrm{~m}, 8 \mathrm{H}), 6.35(\mathrm{~d}$, br, 1H), $5.34(\mathrm{q}, 1 \mathrm{H}), 1.61(\mathrm{~d}, 3 \mathrm{H})$

${ }^{13} \mathrm{C}\left(\mathrm{NMR} 125 \mathrm{MHz}, \mathrm{CDCl}_{3}, 25^{\circ} \mathrm{C}\right): \delta[\mathrm{ppm}]=166.7(\mathrm{~s}), 143.3(\mathrm{~s}), 134.8(\mathrm{~s}), 131.6(\mathrm{~s})$, 128.9 (s), 128.7 (s), 127.6 (s), 127.1 (s), 126.4 (s), 49.4 (s), 21.9 (s); 


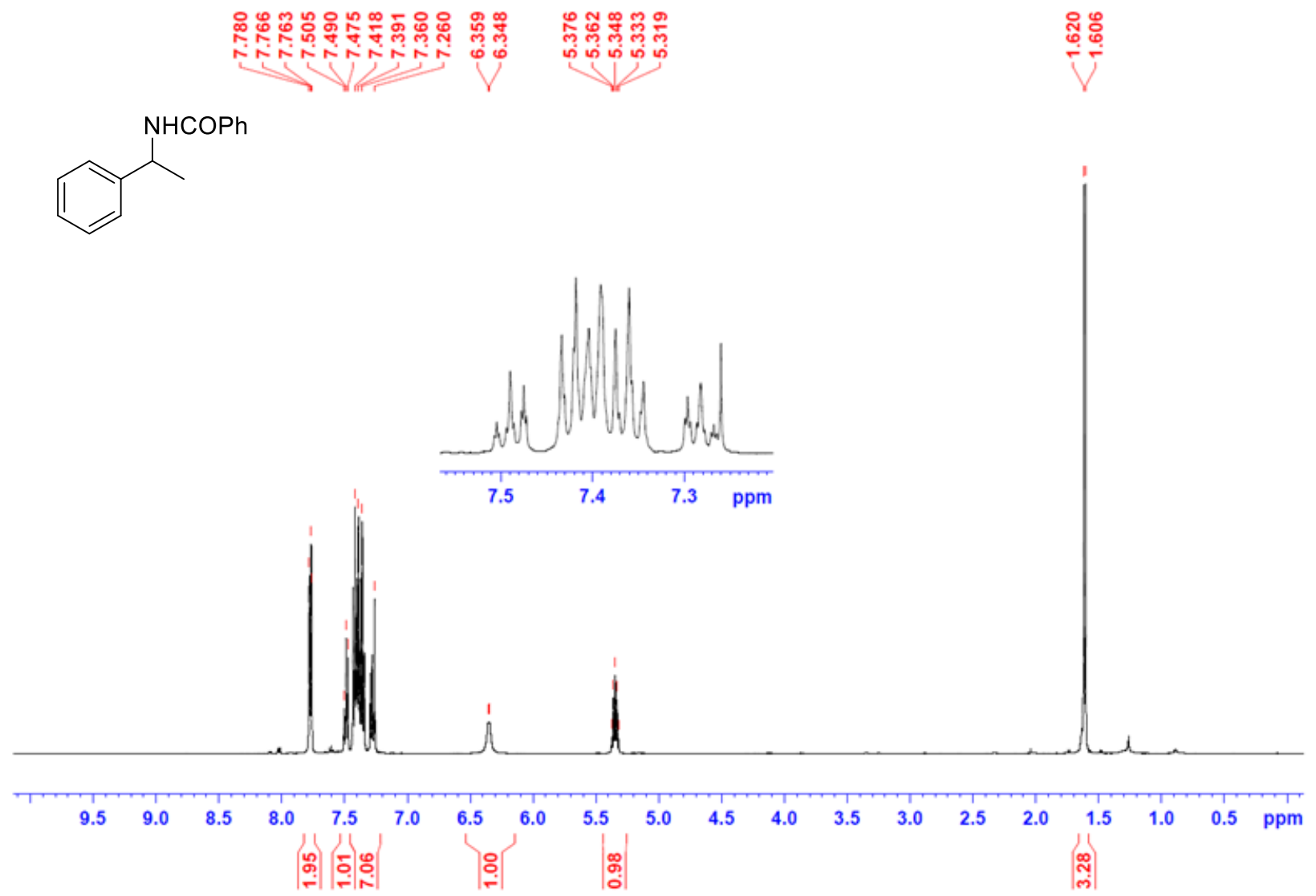

Figure S24. ${ }^{1} \mathrm{H}$ NMR of N-(1-Phenylethyl)benzamide in $\mathrm{CDCl}_{3}$. 


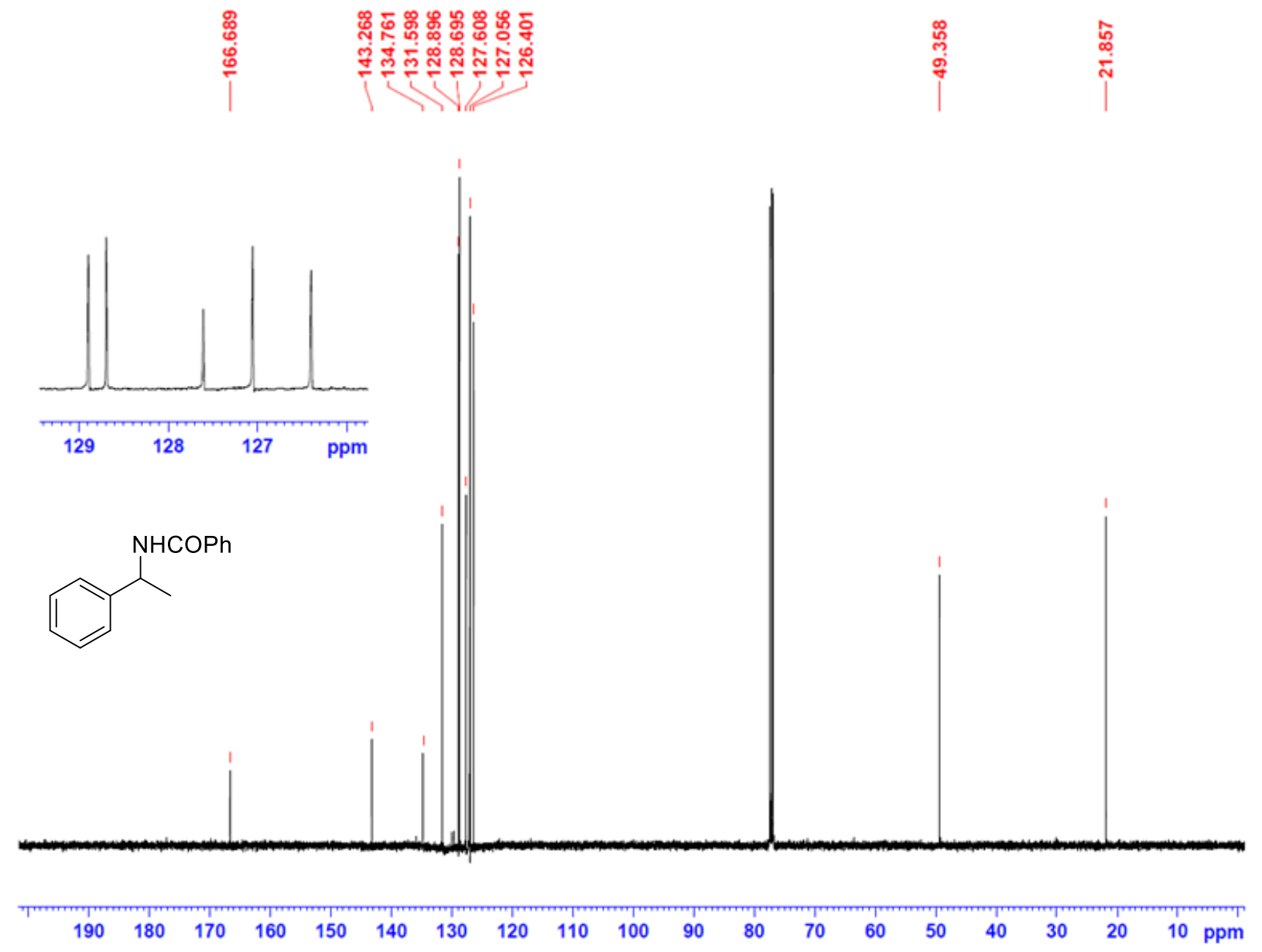

Figure S25. ${ }^{13} \mathrm{C}$ NMR of N-(1-Phenylethyl)benzamide in $\mathrm{CDCl}_{3}$. 


\section{2,3,4,5,6-Pentafluoro-N-(1-phenylethyl)benzamide}<smiles>CC(NC(=O)c1c(F)c(F)c(F)c(F)c1F)c1ccccc1</smiles>

In a glovebox, $\mathrm{Cu}\left(\mathrm{BF}_{4}\right)_{2} \cdot 6 \mathrm{H}_{2} \mathrm{O}(23.0 \mathrm{mg}, 63.0 \mu \mathrm{mol}, 10.0 \mathrm{~mol} \%)$, ligand $\mathbf{A}(30.0 \mathrm{mg}, 63 \mu \mathrm{mol}$, $10.0 \mathrm{~mol} \%)$, 2,3,4,5,6-pentafluorobenzamide $(396 \mathrm{mg}, 1.88 \mathrm{mmol}, 3.00$ equiv), $\mathrm{N}$ Fluorobenzenesulfonimide (593 mg, $1.88 \mathrm{mmol}, 3.00$ equiv) and ethylbenzene ( $80 \mu \mathrm{L}, 13.3 \mathrm{mg}$, $0.625 \mathrm{mmol}, 1.00$ equiv.) were added in this sequence to a $20 \mathrm{~mL}$ scintillation vial equipped with a Teflon-coated stir bar. The vial was sealed with a Teflon-lined vial cap and heated to $110{ }^{\circ} \mathrm{C}$ on a pre-heated vial plate under vigorous stirring (1500 rpm). After $48 \mathrm{~h}$, the vial was taken off the heating block and the mixture was allowed to cool to room temperature. The solvent was removed under reduced pressure. $15 \mathrm{~mL} \mathrm{CHCl}_{3}$ and $2 \mathrm{~g} \mathrm{CaCO}_{3}$ were added into the mixture and the resulting suspension was filtered through Celite. The filtrate was concentrated under vacuum (rotary evaporator) to yield the crude product which was purified by column chromatography using ethyl acetate/petroleum ether (1:9 to $1: 1)$ as eluent. $35 \mathrm{mg}$ (18\% yield) of pure title compound was obtained.

${ }^{1} \mathrm{H}$ NMR $\left(500 \mathrm{MHz}, \mathrm{CDCl}_{3}, 25^{\circ} \mathrm{C}\right): \delta[\mathrm{ppm}]=7.39-7.26(\mathrm{~m}, 5 \mathrm{H}), 6.37(\mathrm{~d}, 1 \mathrm{H}), 5.27(\mathrm{q}$, $1 \mathrm{H}), 1.59(\mathrm{~d}, 3 \mathrm{H})$;

${ }^{13} \mathrm{C}\left(\mathrm{NMR} 125 \mathrm{MHz}, \mathrm{CDCl}_{3}, 25^{\circ} \mathrm{C}\right): \delta[\mathrm{ppm}]=156.6(\mathrm{~s}), 145.2(\mathrm{~m}), 143.3(\mathrm{~m}), 141.9$ (s), $141.4(\mathrm{~m}), 138.8(\mathrm{~m}), 136.7(\mathrm{~m}), 129.0(\mathrm{~s}), 127.9(\mathrm{~s}), 126.2(\mathrm{~s}), 111.7(\mathrm{~m}), 50.1(\mathrm{~s}), 21.6(\mathrm{~s})$;

IR (ATR): ${ }^{\tilde{v}}\left[\mathrm{~cm}^{-1}\right]=3349(\mathrm{~s}), 3076(\mathrm{~s}), 3037(\mathrm{~s}), 2993(\mathrm{~s}), 2362(\mathrm{~s}), 2324(\mathrm{~s}), 1655(\mathrm{~s})$, $1538(\mathrm{~s}), 1514$ (s), 1486 (s), 1450 (s), 1407 (s), 1381 (s), 1323 (s), 1308 (s), 1282 (s), 1248 (s), 1214 (s), 1130 (s), 1117 (s), 1092 (s), 1032 (s), 1017 (s), 992 (s), 947 (s), 907 (s), 809 (s), 791 (s), 764 (s), 734 (s), 698 (s), 649 (s), $614(\mathrm{~s})$;

HRMS: calcd. for $\mathrm{C}_{15} \mathrm{H}_{11} \mathrm{~F}_{5} \mathrm{NO}[\mathrm{M}+\mathrm{H}]^{+}: 316.0761$, found: 316.0740 


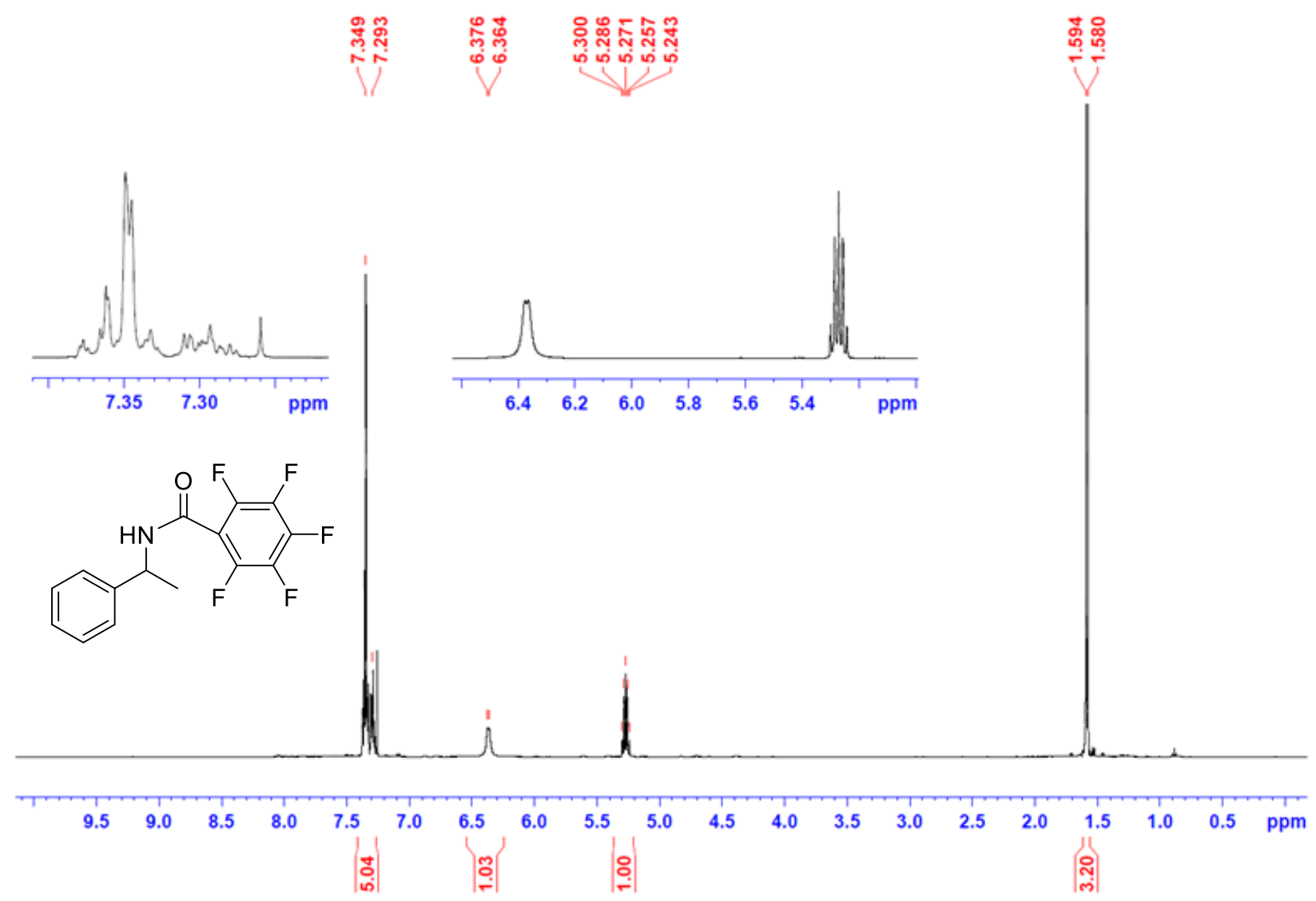

Figure S26. ${ }^{1} \mathrm{H}$ NMR of 2,3,4,5,6-Pentafluoro-N-(1-phenylethyl)benzamide in $\mathrm{CDCl}_{3}$. 

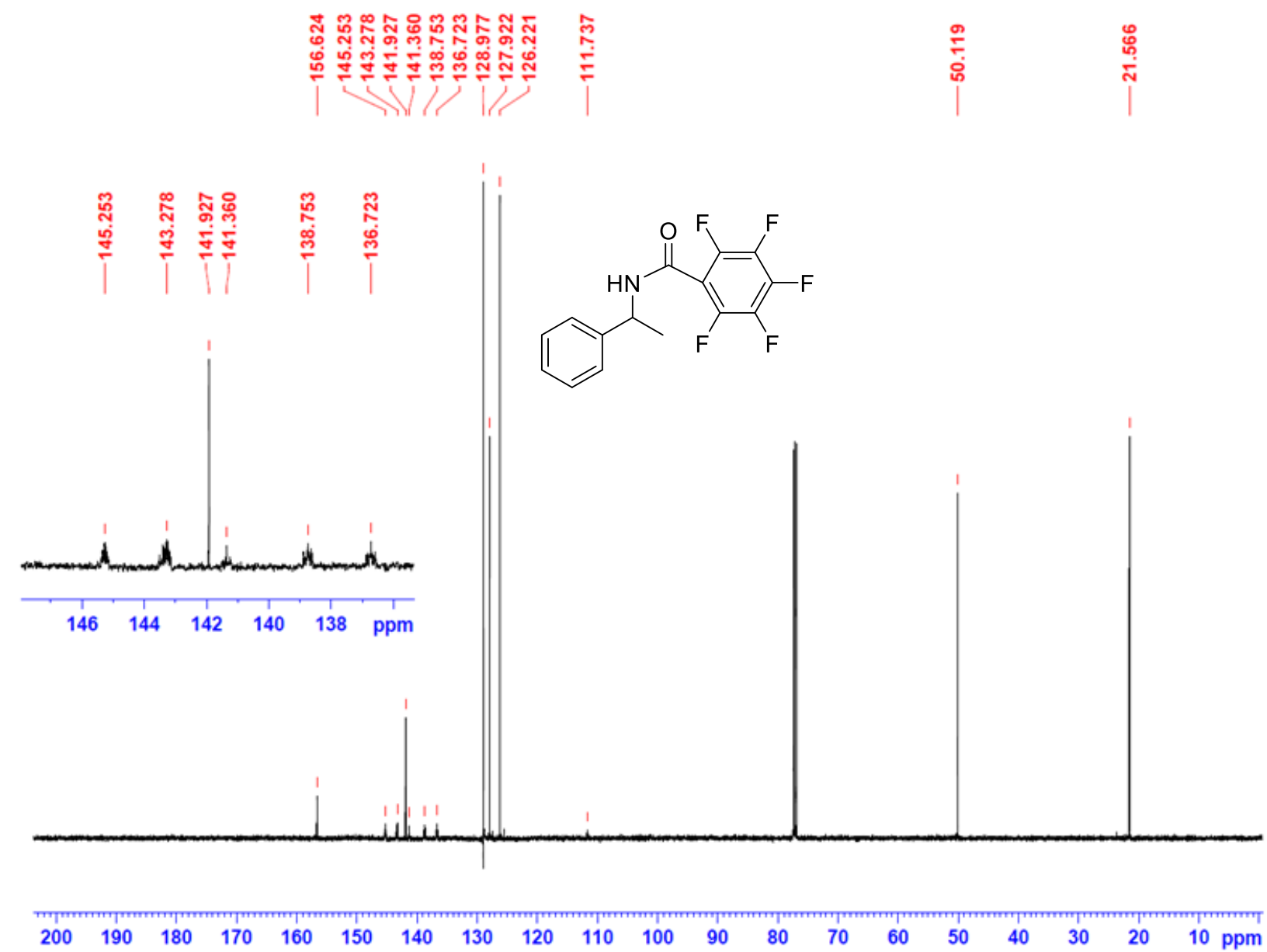

Figure S27. ${ }^{13} \mathrm{C}$ NMR of 2,3,4,5,6-Pentafluoro-N-(1-phenylethyl)benzamide in $\mathrm{CDCl}_{3}$. 


\section{KIE Experiment}

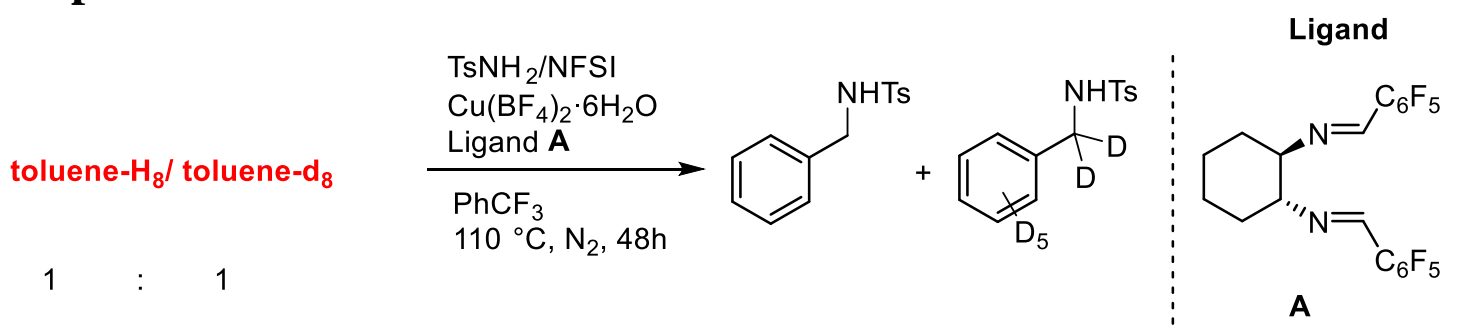

In a glovebox, $\mathrm{Cu}\left(\mathrm{BF}_{4}\right)_{2} \cdot 6 \mathrm{H}_{2} \mathrm{O}(23.0 \mathrm{mg}, 63.0 \mu \mathrm{mol}, 10.0 \mathrm{~mol} \%)$, ligand $\mathbf{A}(30.0 \mathrm{mg}, 63 \mu \mathrm{mol}$, $10.0 \mathrm{~mol} \%$ ), $\mathrm{TsNH}_{2}$ (324 mg, $1.88 \mathrm{mmol}, 3.00$ equiv), N-Fluorobenzenesulfonimide (593 mg, $1.88 \mathrm{mmol}, 3.00$ equiv) toluene $(36 \mu \mathrm{L}, 31.3 \mathrm{mg}, 0.313 \mathrm{mmol}, 0.50$ equiv.) and toluene-d8 (36 $\mu \mathrm{L}, 33.8 \mathrm{mg}, 0.313 \mathrm{mmol}, 0.50$ equiv.) were added in this sequence to a $20 \mathrm{~mL}$ scintillation vial equipped with a Teflon-coated stir bar. The vial was sealed with a Teflon-lined vial cap and heated to $110^{\circ} \mathrm{C}$ on a pre-heated vial plate under vigorous stirring $(1500 \mathrm{rpm})$. After $48 \mathrm{~h}$, the vial was taken off the heating block and the mixture was allowed to cool to room temperature. The solvent was removed under reduced pressure. $15 \mathrm{~mL} \mathrm{CHCl}_{3}$ and $2 \mathrm{~g} \mathrm{CaCO}_{3}$ were added into the mixture and the resulting suspension was filtered through Celite. The filtrate was analyzed for GC-MS. The kinetic isotope effect (KIE) was calculated to $\mathrm{k}_{\mathrm{H}} / \mathrm{k}_{\mathrm{D}} \approx 1.0$ using the intensity of peaks m/z 106 and m/z 107 (products with only C-H bonds) over the intensity of peaks m/z 113, 114, and 115 (products with only C-D bonds; see Table S12). 


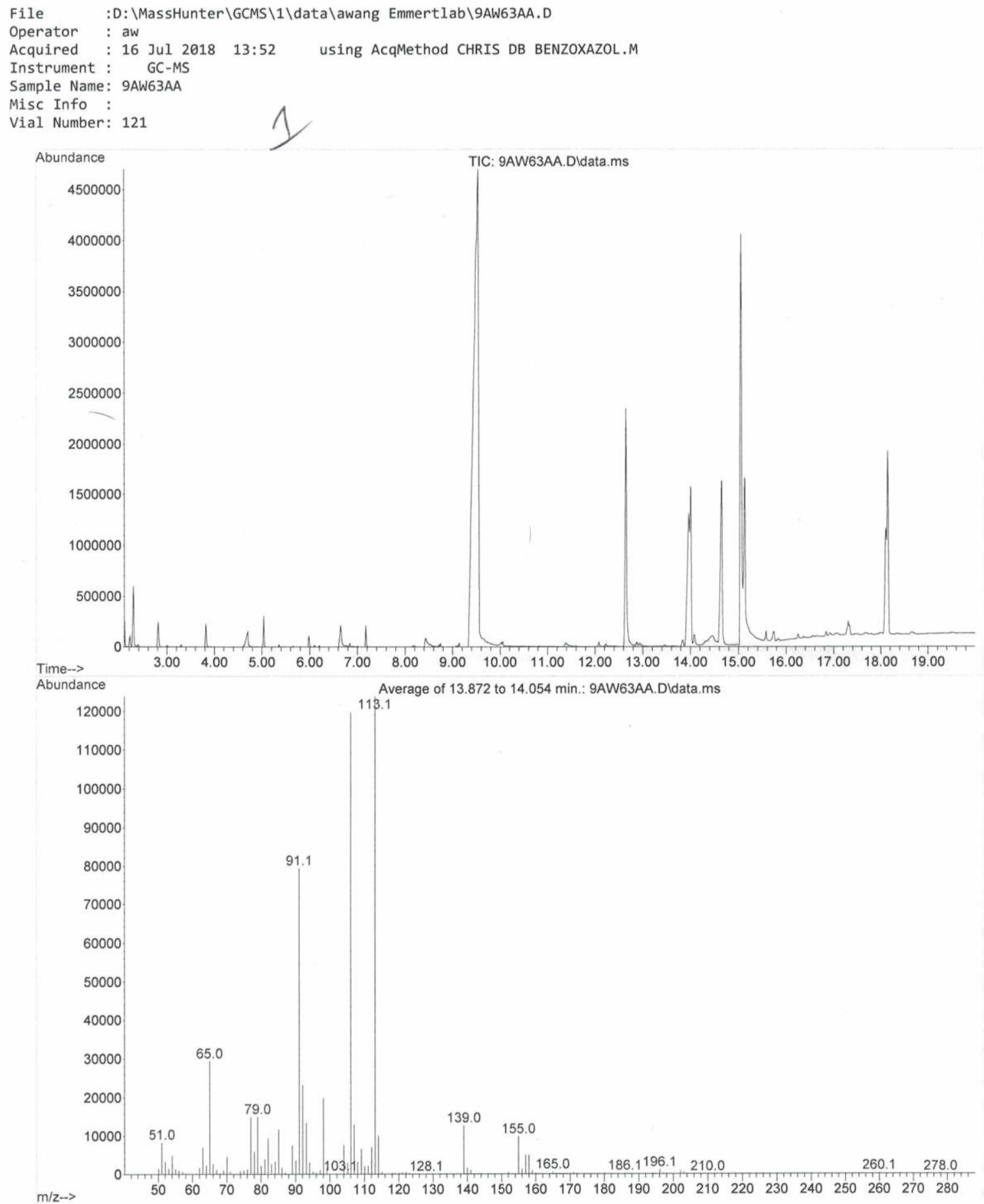

$\mathrm{m} / \mathrm{z}->$

Figure S28. GC-MS Spectrum of KIE Experiment 
File

:D: \MassHunter \GCMS $\backslash 1 \backslash$ data \awang Emmertlab\9AW63AA.D

Operator : aw

Acquired : 16 Jul 2018 13:52 using AcqMethod CHRIS DB BENZOXAZOL.M

Instrument : GC-MS

Sample Name: 9AW63AA

Misc Info :

Vial Number: 121

Abundance

2800000

TIC: 9AW63AA.Dldata.ms

2600000

2400000

2200000

2000000

1800000

1600000

1400000

1200000

1000000

800000

600000 .

400000 .

200000

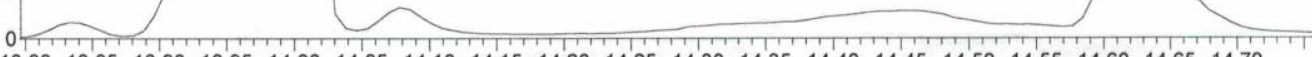

Time-->

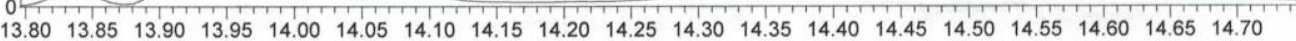
Abundance
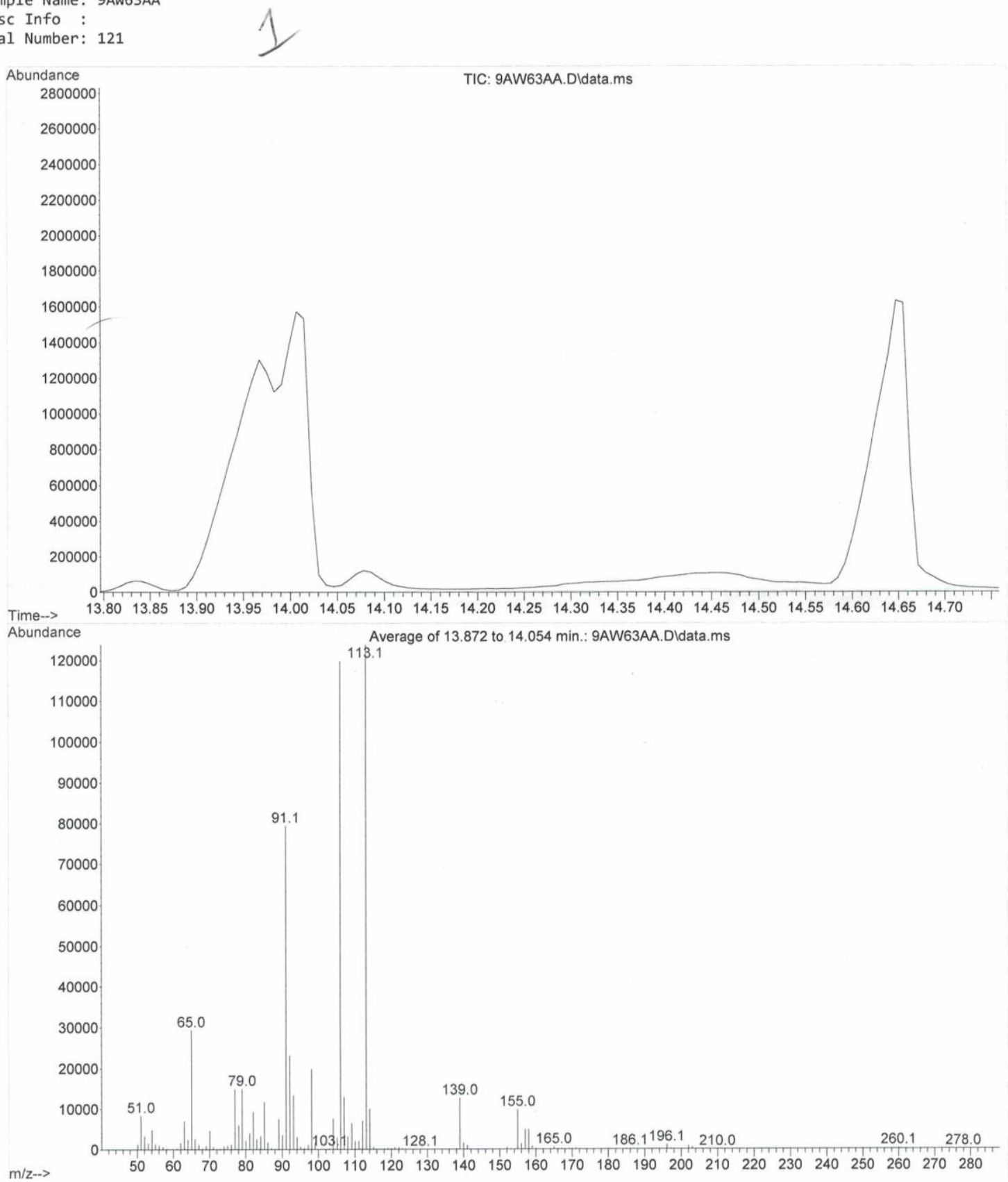

Figure SS29. GC-MS Spectrum KIE Experiment (Zoom in View) 
Table S12. Structures and EIMS Abundance of Reaction Products Incorporating Different Isotopes (H vs. D; ${ }^{12} \mathrm{C}$ vs. ${ }^{13} \mathrm{C}$ ).

\begin{tabular}{|c|c|c|c|}
\hline \multicolumn{1}{|c|}{ Structure } & $\mathbf{m} / \mathbf{z}$ & Abundance & $\begin{array}{c}\text { Total for deuterated/non- } \\
\text { deuterated product }\end{array}$ \\
\hline
\end{tabular}




\section{Decomposition Study of 1-Ethylnaphthalene}

Table S13. 1-Ethylnaphthalene ( $0.125 \mathrm{mmol}, 1.00$ equiv.), $\mathrm{Cu}\left(\mathrm{BF}_{4}\right)_{2} \cdot 6 \mathrm{H}_{2} \mathrm{O}(4.6 \mathrm{mg}, 12.6 \mu \mathrm{mol}, 10.0 \mathrm{~mol} \%)$, ligand A $(6.0 \mathrm{mg}, 12.6 \mu \mathrm{mol}, 10.0 \mathrm{~mol} \%)$, amination reagent 1 (p-Toluenesulfonamide, $64.2 \mathrm{mg}, 0.375 \mathrm{mmol}, 3.00$ equiv.), $\mathrm{N}$-Fluorobenzenesulfonimide (118.3 mg, $0.375 \mathrm{mmol}, 3.00$ equiv.) and $1.5 \mathrm{~mL} \mathrm{PhCF}_{3}, 48 \mathrm{~h}$. Another batch of chemicals were added to run for another 48 hours before stopping the reaction for work out, unless noted as nothing. The reactions were prepared according to the Representative Procedure for Catalytic Benzylic Amination Reactions.

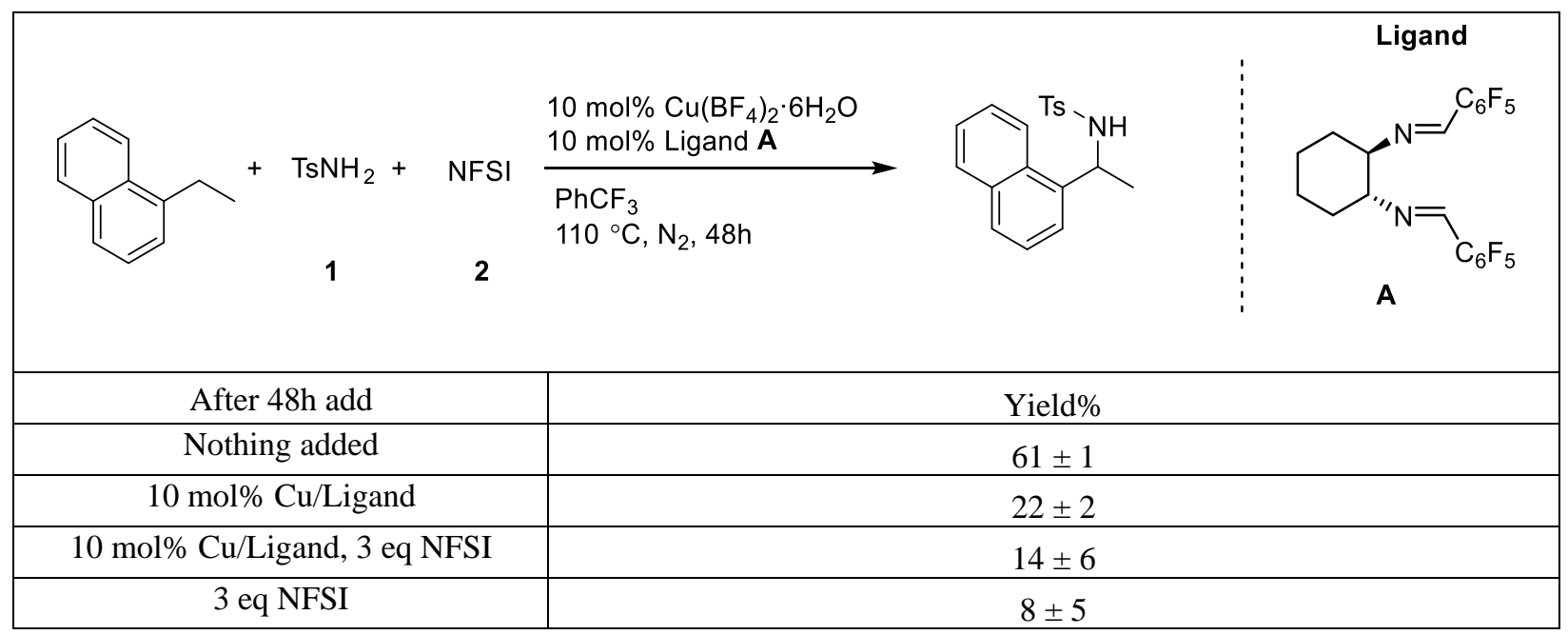




\section{Synthesis of NFSI Amination Product of Toluene}

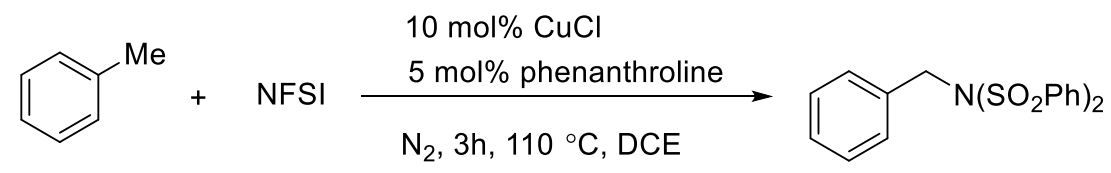

The title compound was synthesized in analogy to a literature known procedure ${ }^{8}$.

A $20 \mathrm{~mL}$ scintillation vial equipped with a Teflon-coated stir bar was charged with toluene (0.212 mL, 2 mmol, 1.0 equiv.), $\mathrm{CuCl}$ (19.80 mg, $0.20 \mathrm{mmol}, 10 \mathrm{~mol} \%)$, NFSI (693.0 mg, 2.20 mmol 1.1 equiv.), 1,10-phenanthroline (9.90 mg, $0.05 \mathrm{mmol}, 5 \mathrm{~mol} \%)$ and $6.0 \mathrm{~mL}$ DCE. The reaction mixture was stirred at $110^{\circ} \mathrm{C}$ for $3 \mathrm{~h}$. Upon completion, the reaction was cooled to room temperature and quenched by addition of $10 \mathrm{~mL} \mathrm{H}_{2} \mathrm{O}$. The mixture was then extracted with $3 \times$ $10 \mathrm{~mL} \mathrm{CH}_{2} \mathrm{Cl}_{2}$. The combined organic extracts were washed with $10 \mathrm{~mL}$ sat. aqueous $\mathrm{NaHCO}_{3}$ solution and $10 \mathrm{~mL}$ brine. The organic layer was dried over $\mathrm{MgSO}_{4}$ and concentrated under reduced pressure to afford a brown solid. The crude product was purified by recrystallization from EtOAc/hexane to afford a white solid (488 $\mathrm{mg}, 63 \%)$.

${ }^{1} \mathrm{H} \mathrm{NMR}\left(500 \mathrm{MHz}, \mathrm{CDCl}_{3}, 25^{\circ} \mathrm{C}\right): \delta[\mathrm{ppm}]=4.94(\mathrm{~s}, 2 \mathrm{H}), 7.20-7.29(\mathrm{~m}, 3 \mathrm{H}), 7.35-7.40$ (m, 2H), 7.40-7.45 (m, 4H), 7.54-7.60 (m, 2H), 7.76-7.80 (m, 4H);

${ }^{13} \mathrm{C}\left(\mathrm{NMR} 125 \mathrm{MHz}, \mathrm{CDCl}_{3}, 25^{\circ} \mathrm{C}\right): \delta[\mathrm{ppm}]=52.6(\mathrm{~s}), 128.3(\mathrm{~s}), 128.6(\mathrm{~s}), 128.9(\mathrm{~s})$, $128.4(\mathrm{~s}), 133.7(\mathrm{~s}), 134.6(\mathrm{~s}), 140.2(\mathrm{~s})$; 


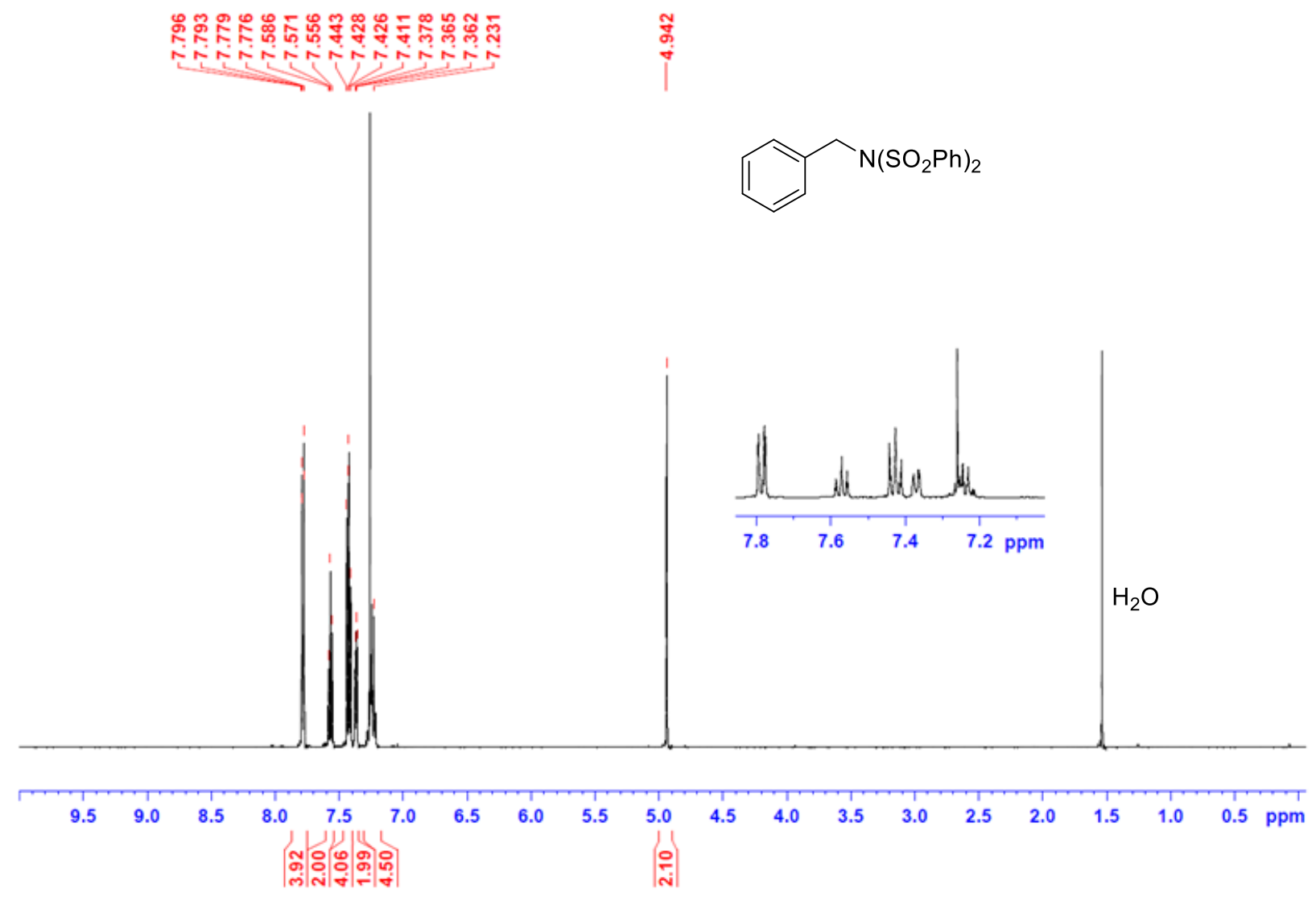

Figure S30. ${ }^{1} \mathrm{H}$ NMR of $N$-Benzyl- $N$-(phenylsulfonyl)benzenesulfonamide in $\mathrm{CDCl}_{3}$. 

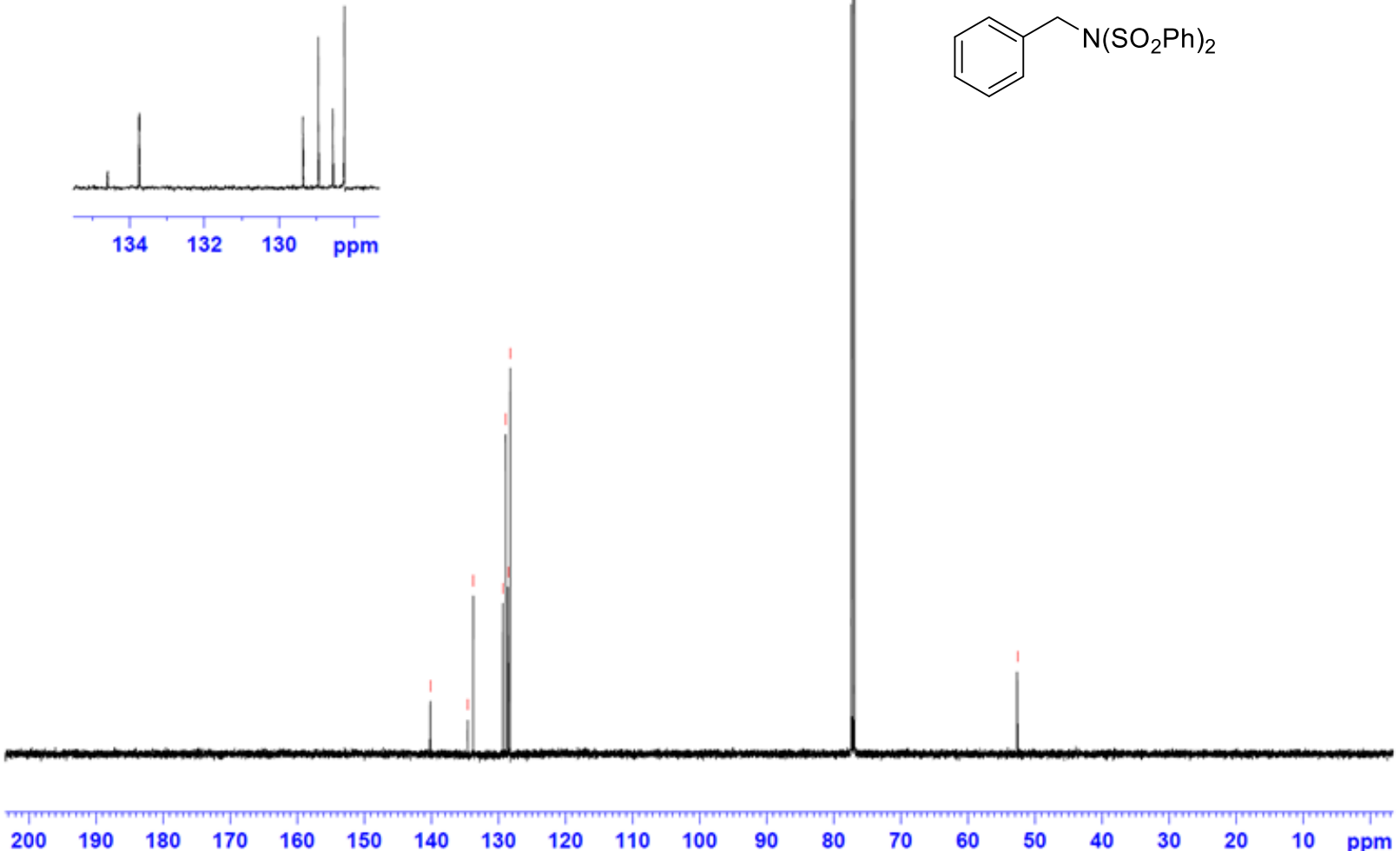

Figure S31. ${ }^{13} \mathrm{C}$ NMR of $N$-Benzyl- $N$-(phenylsulfonyl)benzenesulfonamide in $\mathrm{CDCl}_{3}$. 


\section{Catalytic reaction in the presences of NFSI amination product of toluene $\left[\mathrm{PhCH}_{2}\left(\mathrm{NSO}_{2} \mathbf{P h}\right)_{2}\right]$}

The following reaction was performed to gain insight into the viability of $\mathrm{PhCH}_{2}\left(\mathrm{NSO}_{2} \mathrm{Ph}\right)_{2}$ being an intermediate rather than a side product.

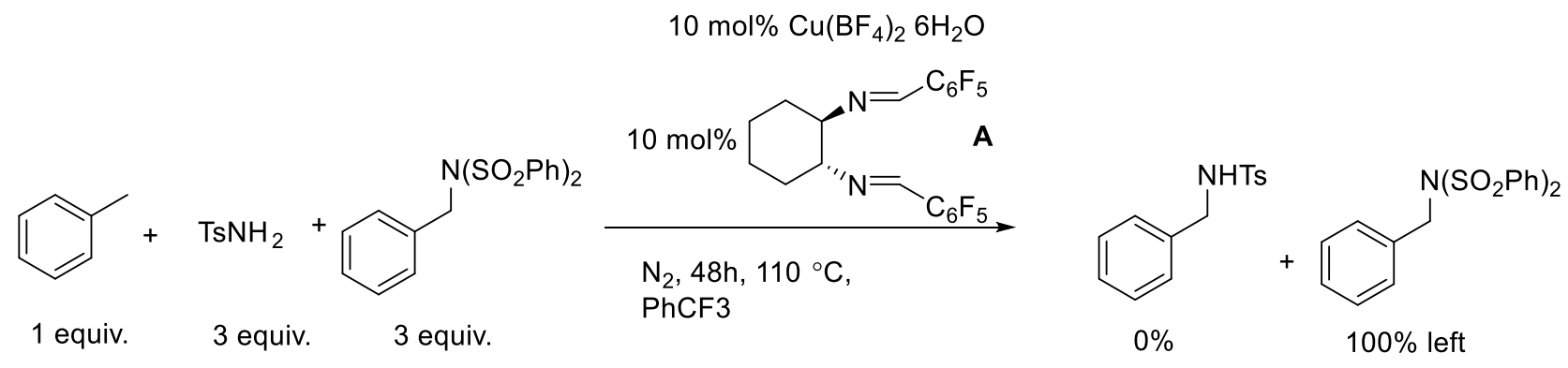

Procedure: In a glovebox, $\mathrm{Cu}\left(\mathrm{BF}_{4}\right)_{2} \cdot 6 \mathrm{H}_{2} \mathrm{O}(4.6 \mathrm{mg}, 12.6 \mu \mathrm{mol}, 10.0 \mathrm{~mol} \%)$, ligand $\mathbf{A}(6.0 \mathrm{mg}$, $12.6 \mu \mathrm{mol}, 10.0 \mathrm{~mol} \%$ ), p-toluenesulfonamide ( $64.2 \mathrm{mg}, 0.375 \mathrm{mmol}, 3.00$ equiv.), $N$-benzyl- $N$ (phenylsulfonyl)benzenesulfonamide (145.1 mg, $0.375 \mathrm{mmol}, 3.00$ equiv.), toluene $(14.0 \mu \mathrm{L}$, $0.125 \mathrm{mmol}, 1.00$ equiv.), and $1.5 \mathrm{~mL}$ benzotrifluoride were added in this sequence to a $4 \mathrm{~mL}$ scintillation vial, equipped with a Teflon-coated stir bar. The vial was sealed with a Teflon-lined vial cap and heated to $110^{\circ} \mathrm{C}$ on a pre-heated vial plate upon vigorous stirring $(1500 \mathrm{rpm})$. After $48 \mathrm{~h}$, the vial was taken off the heating block and the mixture was allowed to cool to room temperature. The solvent was removed under reduced pressure (rotary evaporator). $1.0 \mathrm{~mL}$ $\mathrm{CDCl}_{3}$ was added into the reaction vial, followed by addition of 1,1,2-trichloroethane $(20.0 \mu \mathrm{L}$, $28.8 \mathrm{mg}, 215.9 \mathrm{mmol}$ ) as NMR standard. The resulting mixture was filtered through Celite. ${ }^{1} \mathrm{H}$ $\mathrm{NMR}$ analysis showed that $100 \% \mathrm{PhCH}_{2}\left(\mathrm{NSO}_{2} \mathrm{Ph}\right)_{2}$ remained in the reaction mixture and that no amination product of toluene was formed. This result was supported by GCMS analysis. 


\section{Analysis of Side Products}

\section{Reaction of Toluene}

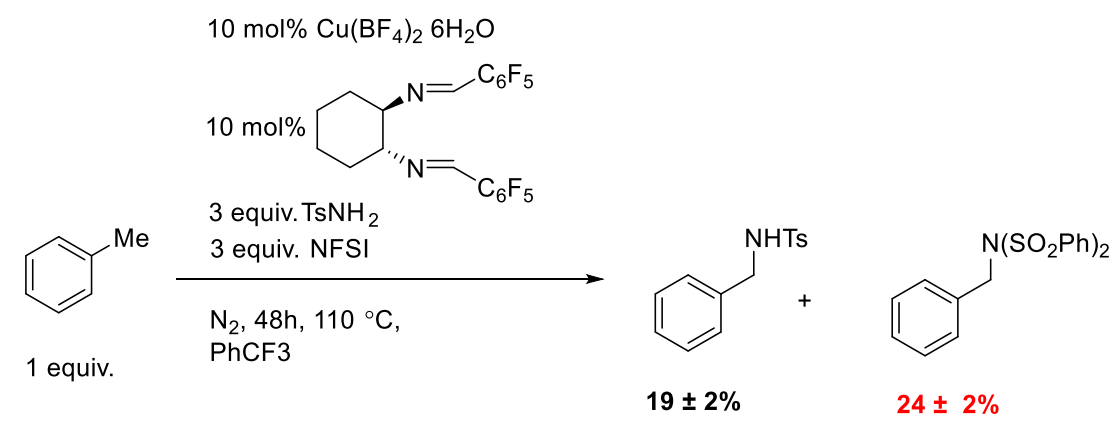

In a glovebox, $\mathrm{Cu}\left(\mathrm{BF}_{4}\right)_{2} \cdot 6 \mathrm{H}_{2} \mathrm{O}(4.6 \mathrm{mg}, 12.6 \mu \mathrm{mol}, 10.0 \mathrm{~mol} \%)$, ligand $\mathbf{A}(6.0 \mathrm{mg}, 12.6 \mu \mathrm{mol}$, $10.0 \mathrm{~mol} \%)$, p-toluenesulfonamide (64.2 $\mathrm{mg}, 0.375 \mathrm{mmol}, 3.00$ equiv.), $N$-benzyl- $N$ (phenylsulfonyl)benzenesulfonamide (145.1 mg, $0.375 \mathrm{mmol}, 3.00$ equiv.), toluene $(14.0 \mu \mathrm{L}$, $0.125 \mathrm{mmol}, 1.00$ equiv.), and $1.5 \mathrm{~mL}$ benzotrifluoride were added in this sequence to a $4 \mathrm{~mL}$ scintillation vial, equipped with a Teflon-coated stir bar. The vial was sealed with a Teflon-lined vial cap and heated to $110{ }^{\circ} \mathrm{C}$ on a pre-heated vial plate upon vigorous stirring (1500 rpm). After $48 \mathrm{~h}$, the vial was taken off the heating block and the mixture was allowed to cool to room temperature. The solvent was removed under reduced pressure (rotary evaporator). $1.0 \mathrm{~mL}$ $\mathrm{CDCl}_{3}$ was added into the reaction vial, followed by addition of 1,1,2-trichloroethane $(20.0 \mu \mathrm{L}$, $28.8 \mathrm{mg}, 215.9 \mathrm{mmol}$ ) as NMR standard. The resulting mixture was filtered through Celite. ${ }^{1} \mathrm{H}$ $\mathrm{NMR}$ analysis showed that $24 \%$ of side product $\mathrm{PhCH}_{2}\left(\mathrm{NSO}_{2} \mathrm{Ph}\right)_{2}$ and $19 \%$ of BnNHTs was formed. This result was supported by GCMS analysis. 


\section{Reaction of 4-Ethyltoluene}

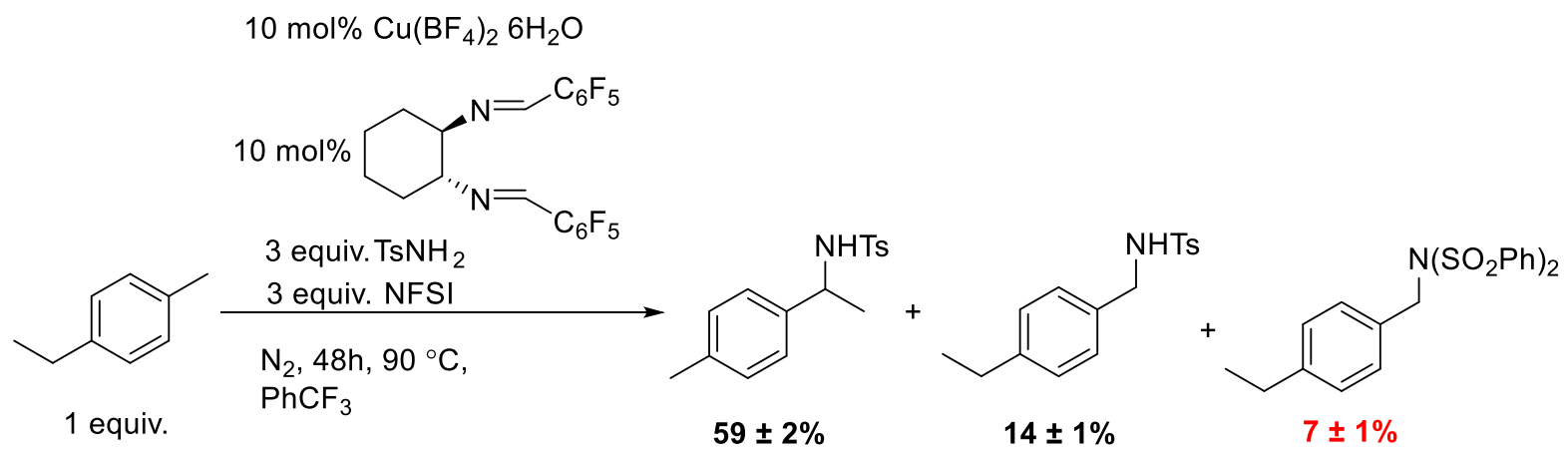

In a glovebox, $\mathrm{Cu}\left(\mathrm{BF}_{4}\right)_{2} \cdot 6 \mathrm{H}_{2} \mathrm{O}(4.6 \mathrm{mg}, 12.6 \mu \mathrm{mol}, 10.0 \mathrm{~mol} \%)$, ligand $\mathbf{A}(6.0 \mathrm{mg}, 12.6 \mu \mathrm{mol}$, $10.0 \mathrm{~mol} \%)$, p-toluenesulfonamide (64.2 $\mathrm{mg}, 0.375 \mathrm{mmol}, 3.00$ equiv.), $N$-benzyl- $N$ (phenylsulfonyl)benzenesulfonamide ( $145.1 \mathrm{mg}, 0.375 \mathrm{mmol}, 3.00$ equiv.), 4-ethyltoluene (16.0 $\mu \mathrm{L}, 0.125 \mathrm{mmol}, 1.00$ equiv.), and $1.5 \mathrm{~mL}$ benzotrifluoride were added in this sequence to a $4 \mathrm{~mL}$ scintillation vial, equipped with a Teflon-coated stir bar. The vial was sealed with a Teflonlined vial cap and heated to $90{ }^{\circ} \mathrm{C}$ on a pre-heated vial plate upon vigorous stirring (1500 rpm). After $48 \mathrm{~h}$, the vial was taken off the heating block and the mixture was allowed to cool to room temperature. The solvent was removed under reduced pressure (rotary evaporator). $1.0 \mathrm{~mL}$ $\mathrm{CDCl}_{3}$ was added into the reaction vial, followed by addition of 1,1,2-trichloroethane $(20.0 \mu \mathrm{L}$, $28.8 \mathrm{mg}, 215.9 \mathrm{mmol}$ ) as NMR standard. The resulting mixture was filtered through Celite.

${ }^{1} \mathrm{H}$ NMR analysis showed $7 \%$ 4- $\mathrm{EtPhCH}_{2}\left(\mathrm{NSO}_{2} \mathrm{Ph}\right)_{2}, 59 \%$ of the secondary $\mathrm{C}-\mathrm{H}$ amination product, and $14 \%$ of primary $\mathrm{C}-\mathrm{H}$ amination product. This result was supported by GCMS analysis. 


\section{TsNHTBS as Potential Amination Reagent}

\section{Preparation of TsNHTBS}

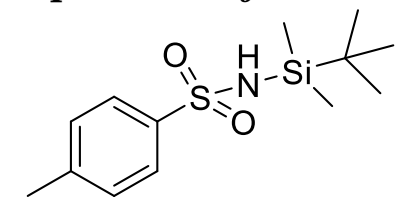

$\mathrm{N}$-(tert-butyldimethylsilyl)-4-

methylbenzenesulfonamide

The title compound was synthesized in analogy to a literature-known procedure?

At room temperature, 4-methylbenzenesulfonamide (5.70 g, $33.0 \mathrm{mmol}, 1.00$ equiv.) was dissolved in dry THF $(40 \mathrm{~mL})$ in a round bottom flask following with the addition of trimethylamine (10.5 mL, $72.4 \mathrm{mmol}, 2.20$ equiv.) under $\mathrm{N}_{2}$. The mixture was cooled to $0{ }^{\circ} \mathrm{C}$. A solution of tert-butyldimethylsilyl chloride ( $6.44 \mathrm{~g}, 41.3 \mathrm{mmol}, 1.25$ equiv) in toluene $(7.4 \mathrm{~mL})$ was added dropwise into the reaction. The mixture was allowed to warm up to room temperature and stirred for $2 \mathrm{~h}$, then stirred at $50{ }^{\circ} \mathrm{C}$ for $48 \mathrm{~h}$. The reaction mixture was then cooled to $25^{\circ} \mathrm{C}$ and the solid was removed by filtration and washed with $\mathrm{Et}_{2} \mathrm{O}(2 \times 50 \mathrm{~mL})$. The organic phases were combined and the solvent was removed under reduced pressure overnight to obtain an offwhite solid crude product. The pure title compound was obtained by washing off impurities using distilled hexane $(6.8 \mathrm{~g}, 72 \%)$.

${ }^{1} \mathrm{H}$ NMR $\left.\left(500 \mathrm{MHz}, \mathrm{CDCl}_{3}, 25^{\circ} \mathrm{C}\right): \delta[\mathrm{ppm}]=\right) \delta 7.72-7.77(\mathrm{~m}, 2 \mathrm{H}), 7.24-7.30(\mathrm{~m}, 2 \mathrm{H})$, $4.26(\mathrm{~s}, 1 \mathrm{H}), 2.42(\mathrm{~s}, 3 \mathrm{H}), 0.89(\mathrm{~s}, 3 \mathrm{H}), 0.90(\mathrm{~s}, 9 \mathrm{H}), 0.22(\mathrm{~s}, 6 \mathrm{H})$

${ }^{13} \mathrm{C}\left(\mathrm{NMR} 125 \mathrm{MHz}, \mathrm{CDCl}_{3}, 25^{\circ} \mathrm{C}\right): \delta[\mathrm{ppm}]=142.8$ (s), 141.1 (s), 129.6 (s), 126.3 (s), $25.9(\mathrm{~s}), 21.6(\mathrm{~s}), 17.4(\mathrm{~s}),-4.3(\mathrm{~s})$; 


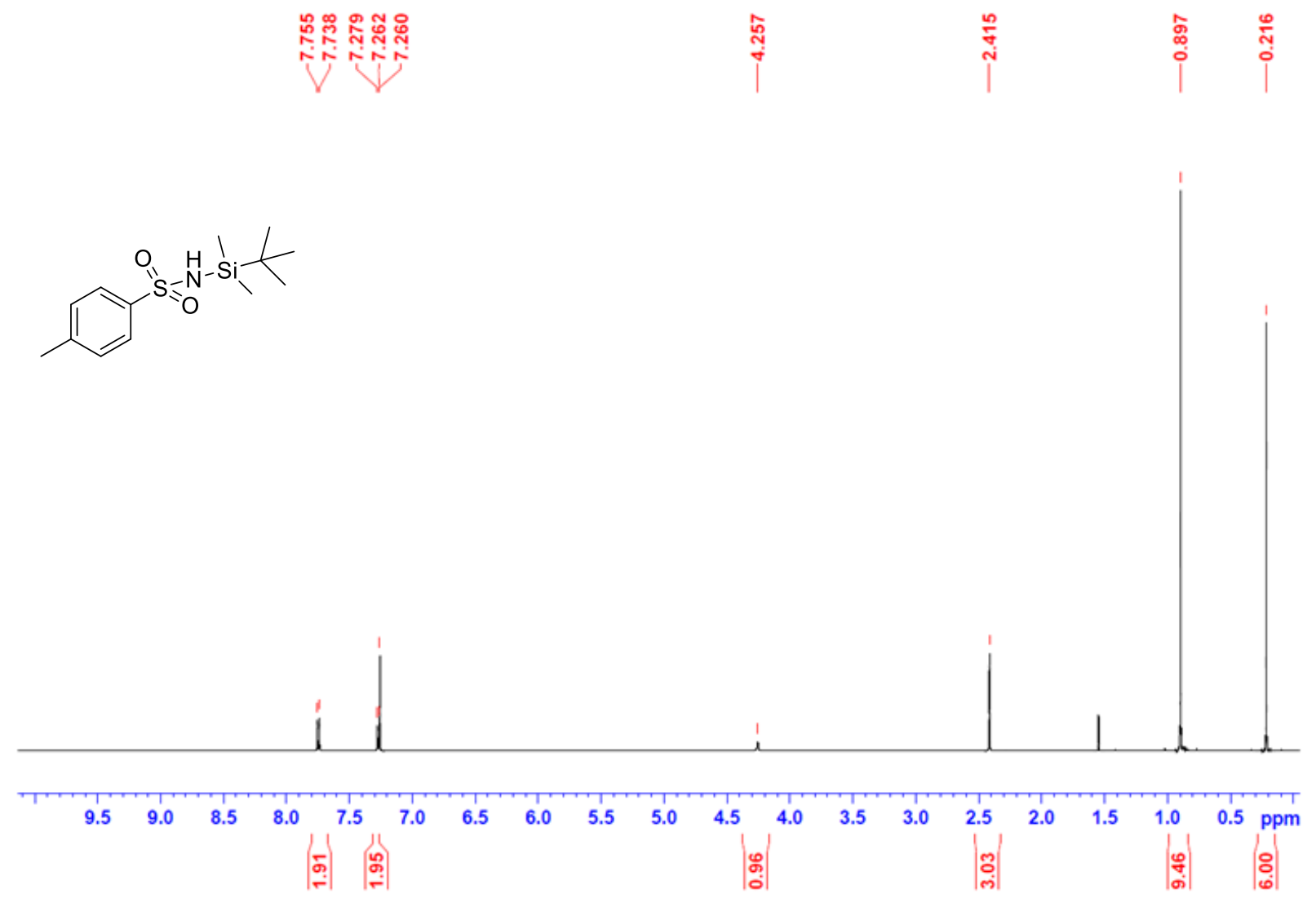

Figure S32. ${ }^{1} \mathrm{H}$ NMR of TsNHTBS in $\mathrm{CDCl}_{3}$. 


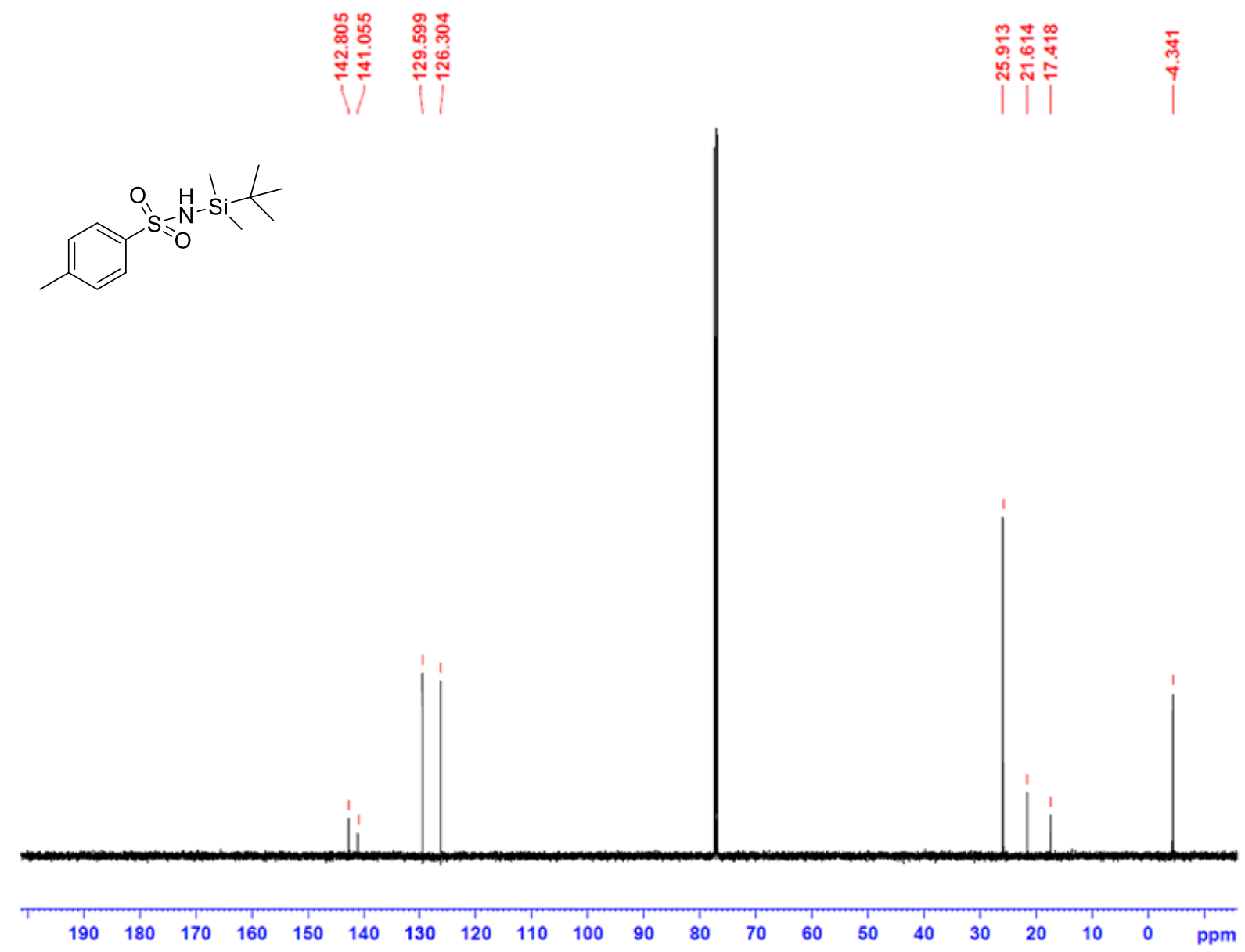

Figure S33. ${ }^{13} \mathrm{C}$ NMR of TsNHTBS in $\mathrm{CDCl}_{3}$. 


\section{Catalytic Reactions with TsNHTBS}

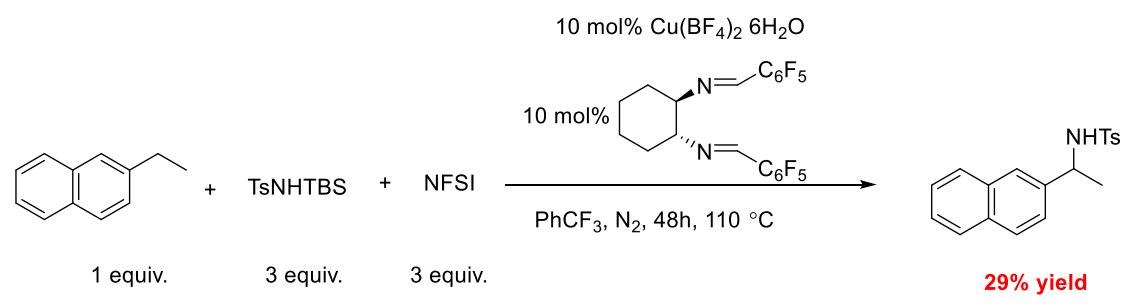

In a glovebox, $\mathrm{Cu}\left(\mathrm{BF}_{4}\right)_{2} \cdot 6 \mathrm{H}_{2} \mathrm{O}(4.6 \mathrm{mg}, 12.6 \mu \mathrm{mol}, 10.0 \mathrm{~mol} \%)$, ligand $\mathbf{A}(6.0 \mathrm{mg}, 12.6 \mu \mathrm{mol}$, $10.0 \mathrm{~mol} \%$ ), TsNHTBS (107 mg, $0.375 \mathrm{mmol}, 3.00$ equiv.), NFSI (118 mg, $0.375 \mathrm{mmol}, 3.00$ equiv.), 2-ethylnaphathlene (20 $\mu \mathrm{L}, 19.5 \mathrm{mg}, 0.125 \mathrm{mmol}, 1.00$ equiv.) and $1.5 \mathrm{~mL}$ benzotrifluoride were added in this sequence to a $4 \mathrm{~mL}$ scintillation vial, equipped with a Tefloncoated stir bar. The vial was sealed with a Teflon-lined vial cap and heated to $110{ }^{\circ} \mathrm{C}$ on a preheated vial plate under vigorous stirring $(1500 \mathrm{rpm})$. After stirring the mixture vigorously for the dedicated reaction time, the vial was taken off the heating block and the mixture was allowed to cool to room temperature. The solvent was removed under reduced pressure (rotary evaporator). 1,1,2-trichloroethane $(20.0 \mu \mathrm{L}, 28.8 \mathrm{mg}, 215.9 \mathrm{mmol})$ was added into the reaction vial as NMR standard followed by addition of $1.0 \mathrm{~mL} \mathrm{CDCl} 3$. The resulting mixture was filtered through Celite. The filtrate was analyzed by quantitative ${ }^{1} \mathrm{H}$ NMR and GC-MS.

The low (29\%) yield (compared with 61\% yield under standard conditions) suggests that sequestering fluoride in the reaction mixture does not contribute to product formation. 


\section{Radical Trap Experiments}

\section{TEMPO}

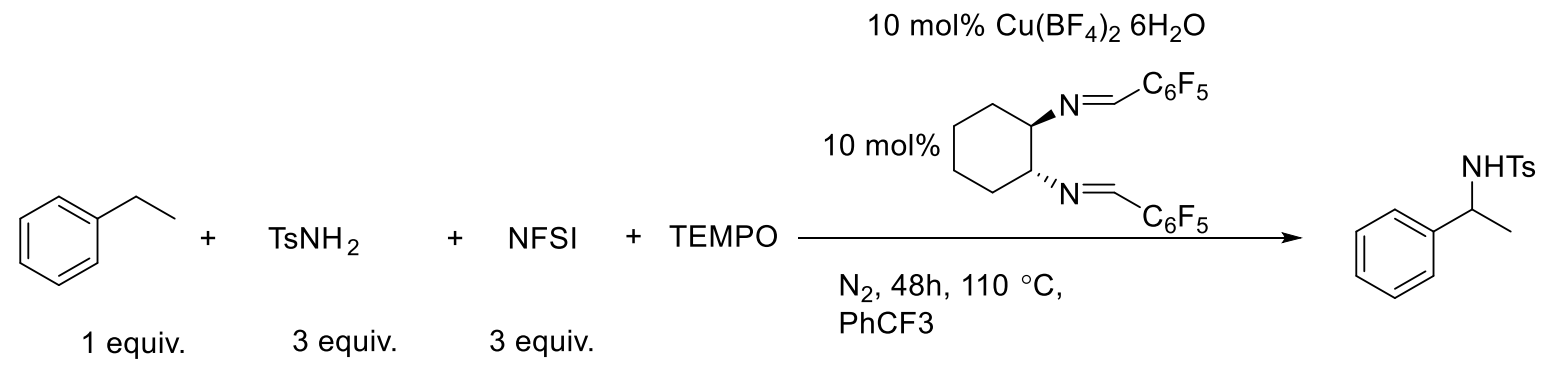

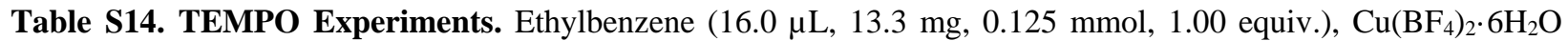
(4.6 mg, $12.6 \mu \mathrm{mol}, 10.0 \mathrm{~mol} \%)$, ligand $\mathbf{A}(12.6 \mu \mathrm{mol}, 10.0 \mathrm{~mol} \%)$, amination reagent $\mathbf{1},(64.7 \mathrm{mg}, 0.375 \mathrm{mmol}$, 3.00 equiv.), $N$-Fluorobenzenesulfonimide ( $118 \mathrm{mg}, 0.375 \mathrm{mmol}, 3.00$ equiv.) TEMPO and $1.5 \mathrm{~mL}$ benzotrifluoride $110{ }^{\circ} \mathrm{C}, 48 \mathrm{~h}$. The reactions were prepared for GC-MS and NMR analysis according to the Representative Procedure for Catalytic Benzylic Amination Reactions.

\begin{tabular}{|l|l|l|l|}
\hline TEMPO & Yield of Amination product & TEMPO Adduct & Remaining Substrate \\
\hline $10 \mathrm{~mol} \%$ & $48.4 \%$ & Not detected & $6.1 \%$ \\
\hline $100 \mathrm{~mol} \%$ & $0 \%$ & Not detected & $0 \%$ \\
\hline $400 \mathrm{~mol} \%$ & $0 \%$ & Not detected & $0 \%$ \\
\hline
\end{tabular}




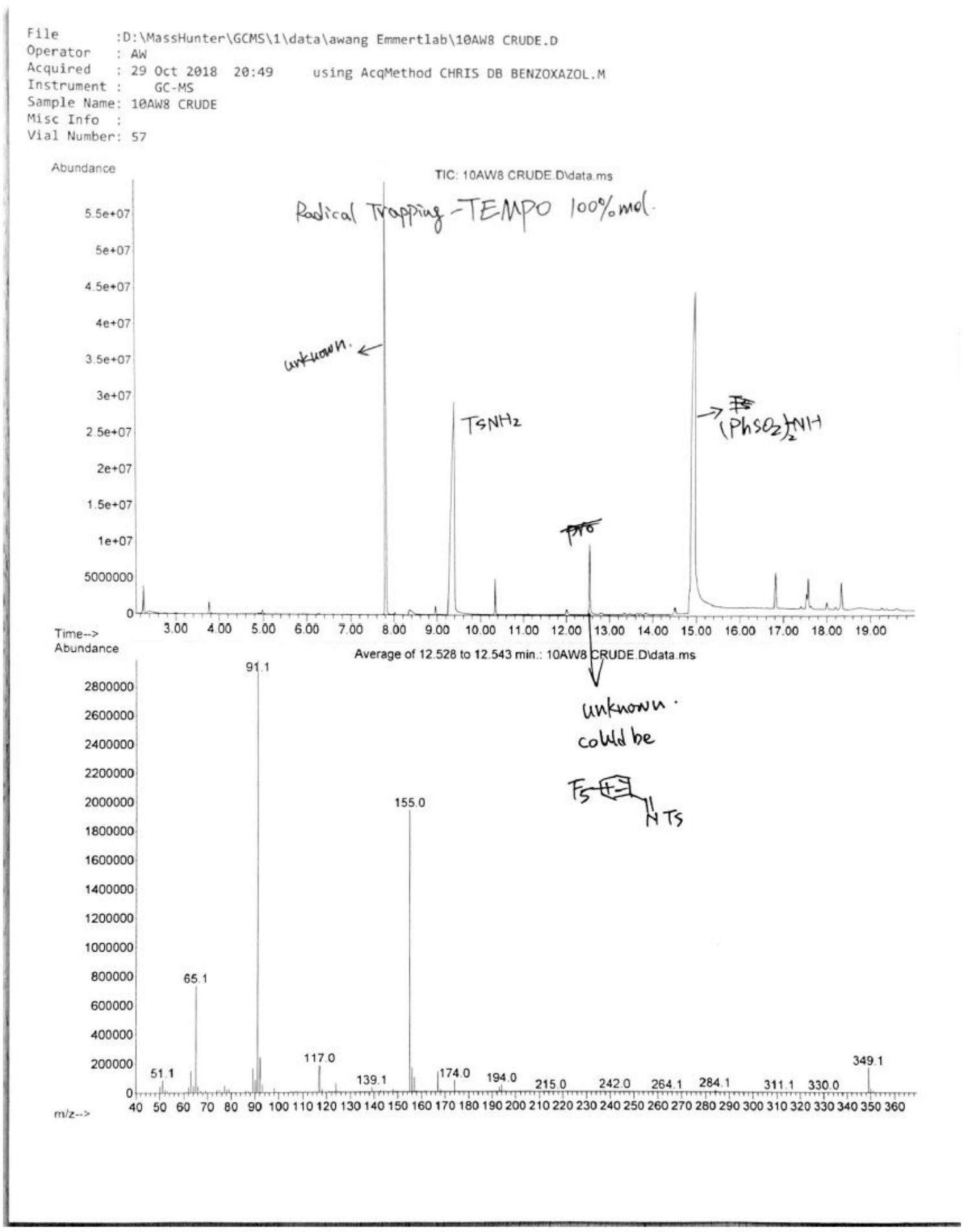

Figure S34. A GC-MS Spectrum for a $48 \mathrm{~h}$ Radical Trapping Experiment using TEMPO 


\section{$\mathrm{BrCCl}_{3}$}

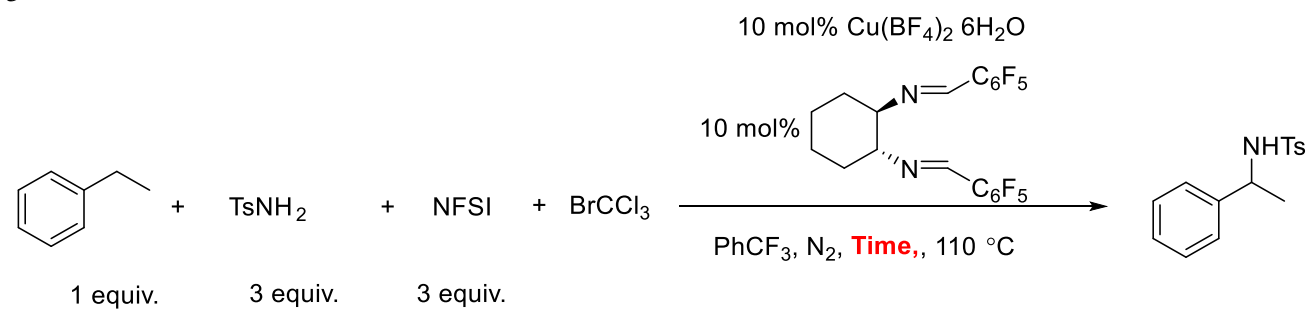

Table S15. BrCCl 3 Experiments. Ethylbenzene ( $16.0 \mu \mathrm{L}, 13.3 \mathrm{mg}, 0.125 \mathrm{mmol}, 1.00$ equiv.), $\mathrm{Cu}\left(\mathrm{BF}_{4}\right)_{2} \cdot 6 \mathrm{H}_{2} \mathrm{O}(4.6$ $\mathrm{mg}, 12.6 \mu \mathrm{mol}, 10.0 \mathrm{~mol} \%)$, ligand A $(12.6 \mu \mathrm{mol}, 10.0 \mathrm{~mol} \%)$, amination reagent 1, $(64.7 \mathrm{mg}, 0.375 \mathrm{mmol}, 3.00$ equiv.), N-Fluorobenzenesulfonimide (118 mg, $0.375 \mathrm{mmol}, 3.00$ equiv.) $\mathrm{BrCCl}_{3}$ and $1.5 \mathrm{~mL}$ benzotrifluoride 110 ${ }^{\circ} \mathrm{C}$. The reactions were prepared for GC-MS and NMR analysis according to the Representative Procedure for Catalytic Benzylic Amination Reactions.

\begin{tabular}{|l|l|l|l|l|}
\hline Amount of BrCCl 3 & Reaction Time & $\begin{array}{l}\text { NMR Yield of } \\
\text { Amination Product }\end{array}$ & Bromination Product & $\begin{array}{l}\text { Remaining } \\
\text { Substrate by NMR }\end{array}$ \\
\hline $10 \mathrm{~mol} \%$ & $15 \mathrm{~min}$ & $8.6 \%$ & Not detected & $90.6 \%$ \\
\hline $100 \mathrm{~mol} \%$ & $15 \mathrm{~min}$ & $10.6 \%$ & Not detected & $86.3 \%$ \\
\hline $400 \mathrm{~mol} \%$ & $15 \mathrm{~min}$ & $18.4 \%$ & Not detected & $78.3 \%$ \\
\hline $10 \mathrm{~mol} \%$ & $1 \mathrm{~h}$ & $38.3 \%$ & Not detected & $37.9 \%$ \\
\hline $100 \mathrm{~mol} \%$ & $1 \mathrm{~h}$ & $51.2 \%$ & Not detected & $13.9 \%$ \\
\hline $400 \mathrm{~mol} \%$ & $1 \mathrm{~h}$ & $61.3 \%$ & Not detected & $10.8 \%$ \\
\hline $10 \mathrm{~mol} \%$ & $48 \mathrm{~h}$ & $74.3 \%$ & Not detected & $5.2 \%$ \\
\hline $100 \mathrm{~mol} \%$ & $48 \mathrm{~h}$ & $70.4 \%$ & Not detected & $3.2 \%$ \\
\hline $400 \mathrm{~mol} \%$ & $48 \mathrm{~h}$ & $51.8 \%$ & Not detected & $0 \%$ \\
\hline
\end{tabular}
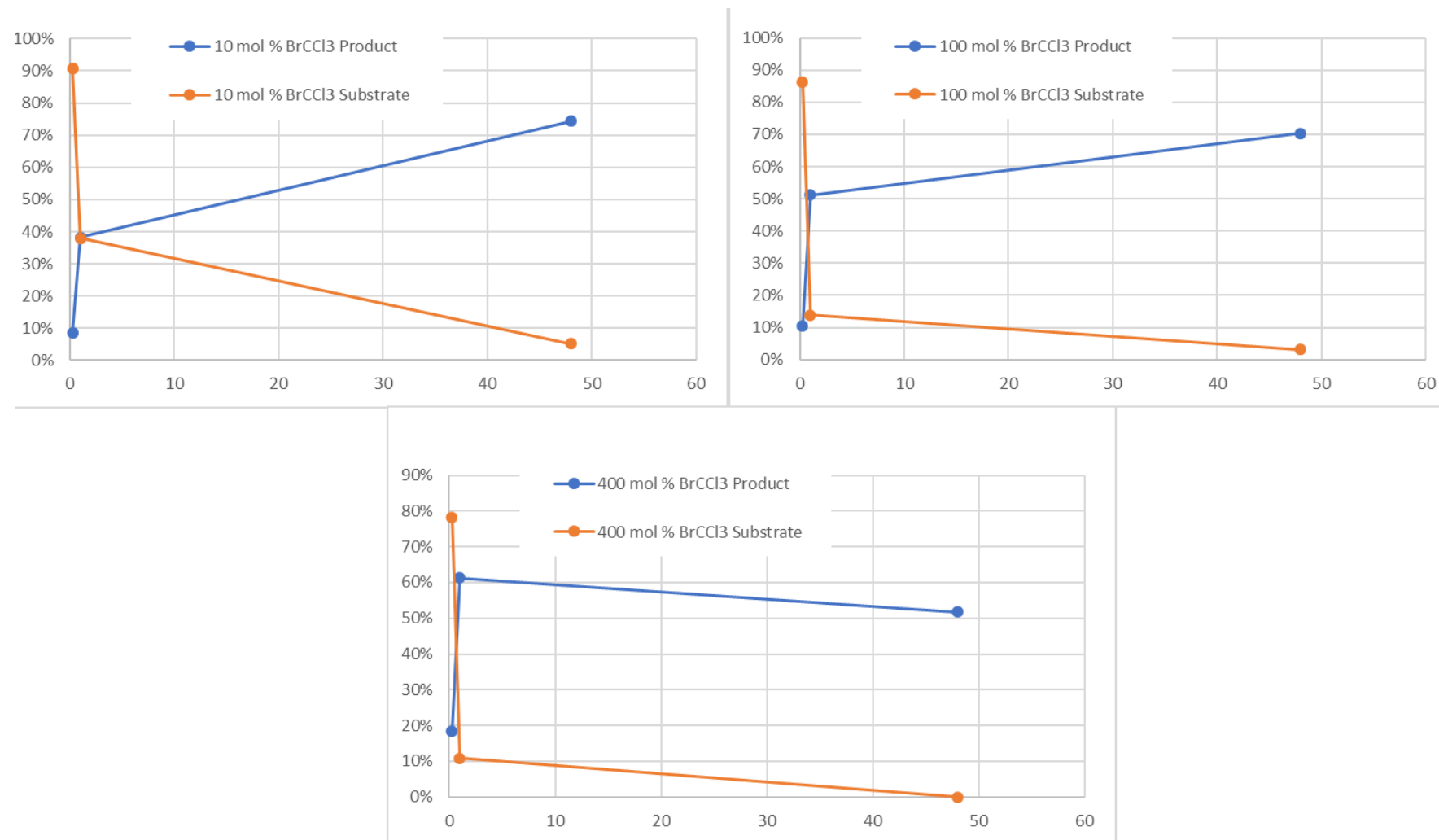

Figure S35. Time courses of reactions with different amounts of $\mathrm{BrCCl}_{3}$ added. 


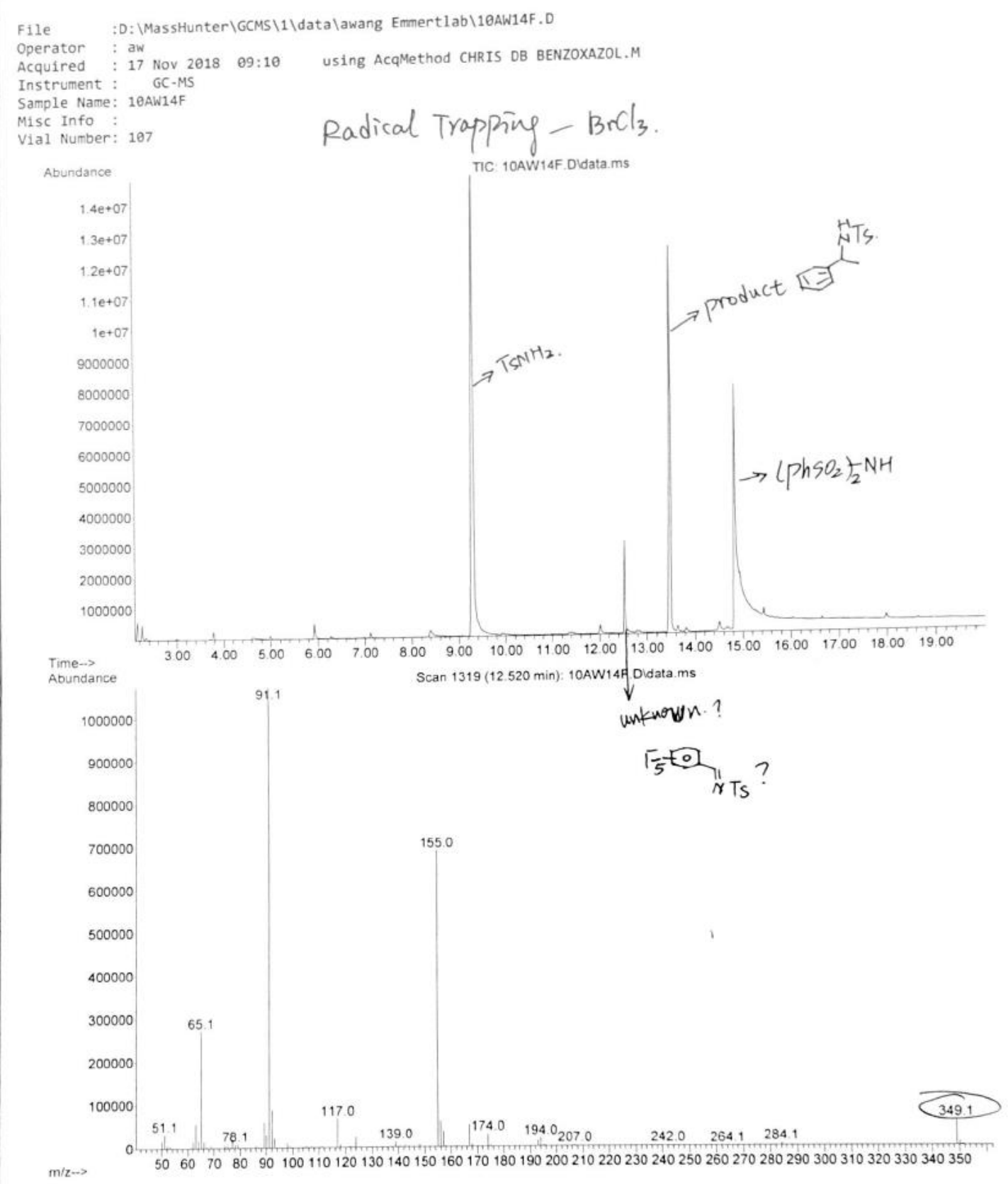

Figure S36. A GC-MS Spectrum for a $48 \mathrm{~h}$ Radical Trapping Experiment using $\mathrm{BrCCl}_{3}$ showing no new compound formed. 


\section{Reaction in Air}

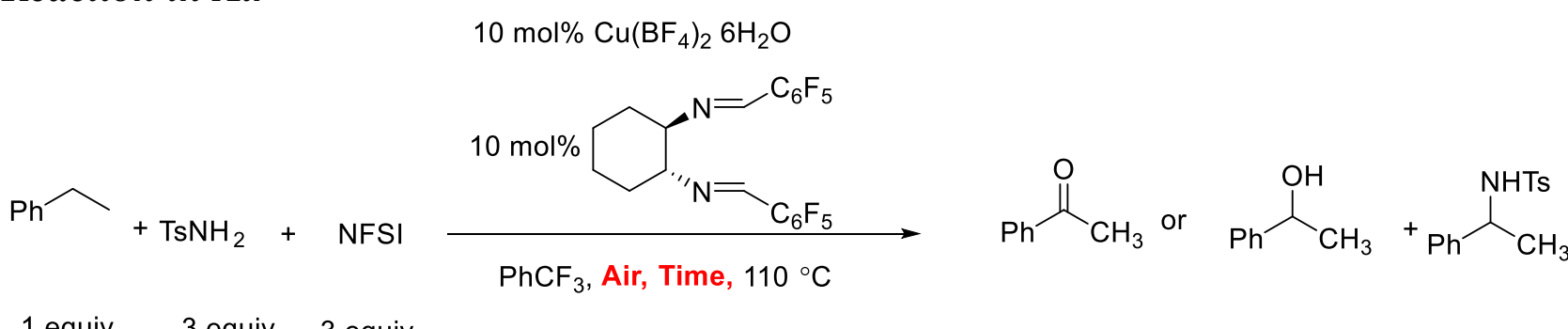

1 equiv. $\quad 3$ equiv. 3 equiv.

Table S16. Air Experiments. Ethylbenzene ( $16.0 \mu \mathrm{L}, 13.3 \mathrm{mg}, 0.125 \mathrm{mmol}, 1.00$ equiv.), $\mathrm{Cu}\left(\mathrm{BF}_{4}\right)_{2} \cdot 6 \mathrm{H}_{2} \mathrm{O}(4.6 \mathrm{mg}$, $12.6 \mu \mathrm{mol}, 10.0 \mathrm{~mol} \%)$, ligand A (12.6 $\mu \mathrm{mol}, 10.0 \mathrm{~mol} \%)$, amination reagent $1,(64.7 \mathrm{mg}, 0.375 \mathrm{mmol}, 3.00$ equiv.), $\mathrm{N}$-Fluorobenzenesulfonimide ( $118 \mathrm{mg}, 0.375 \mathrm{mmol}, 3.00$ equiv.) TEMPO and $1.5 \mathrm{~mL}$ benzotrifluoride $110{ }^{\circ} \mathrm{C}, 48 \mathrm{~h}$ under air. The reactions were prepared for GC-MS and NMR analysis according to the Representative Procedure for Catalytic Benzylic Amination Reactions.

\begin{tabular}{|l|l|l|l|}
\hline Reaction Time & $\begin{array}{l}\text { Oxidation Product (alcohol } \\
\text { or ketone) }\end{array}$ & $\begin{array}{l}\text { NMR Yield of Amination } \\
\text { Product }\end{array}$ & Remaining Substrate \\
\hline $15 \mathrm{~min}$ & Not detected & $5.8 \%$ & $94.7 \%$ \\
\hline $1 \mathrm{~h}$ & Not detected & $28.8 \%$ & $9.8 \%$ \\
\hline $48 \mathrm{~h}$ & Not detected & $60.3 \%$ & $10.4 \%$ \\
\hline
\end{tabular}

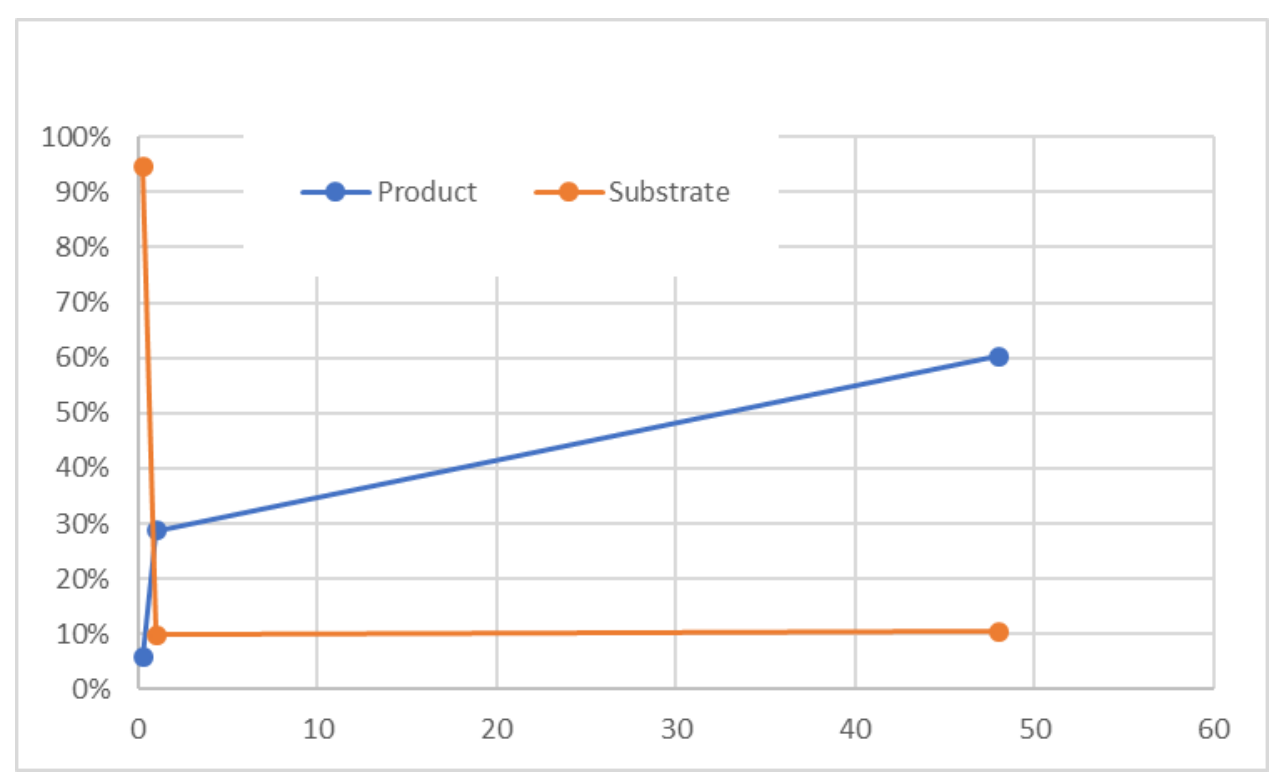

Figure S37. Time Course of Reaction in Air. 


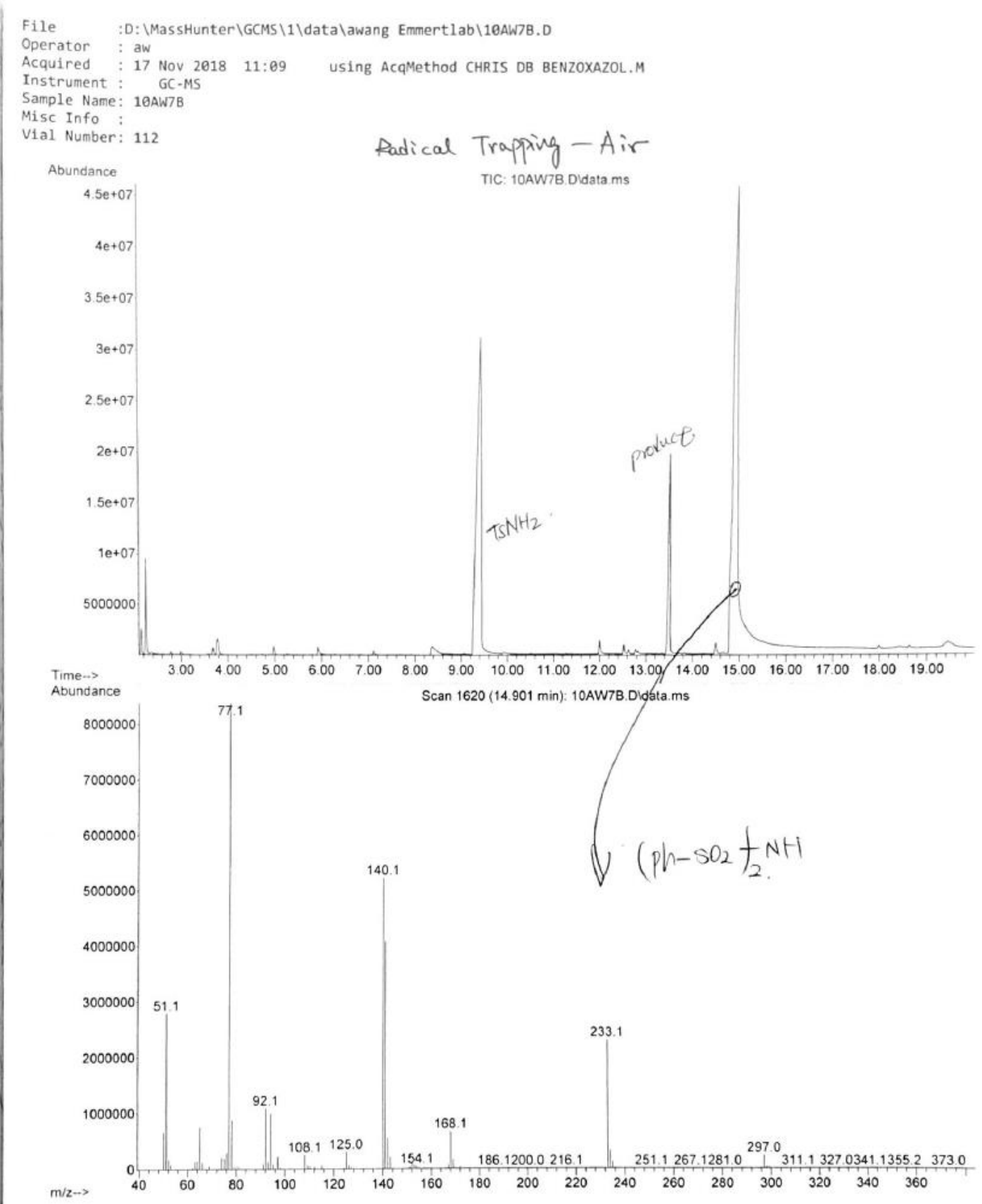

Figure S38. A GC-MS Spectrum for a reaction in air after $48 \mathrm{~h}$, showing no new product. 


\section{References}

1. Milne, J. E.; Storz, T.; Colyer, J. T.; Thiel, O. R.; Dilmeghani Seran, M.; Larsen, R. D.; Murry, J. A., Iodide-Catalyzed Reductions: Development of a Synthesis of Phenylacetic Acids. The Journal of Organic Chemistry 2011, 76 (22), 9519-9524.

2. Taylor, J. G.; Whittall, N.; Hii, K. K., Copper-Catalyzed Intermolecular Hydroamination of Alkenes. Organic Letters 2006, 8 (16), 3561-3564.

3. Zhang, J.; Yang, C.-G.; He, C., Gold(I)-Catalyzed Intra- and Intermolecular Hydroamination of Unactivated Olefins. Journal of the American Chemical Society 2006, 128 (6), 1798-1799.

4. $\quad$ Das, B. G.; Nallagonda, R.; Ghorai, P., Direct Substitution of Hydroxy Group of $\pi$ Activated Alcohols with Electron-Deficient Amines Using Re2O7 Catalyst. The Journal of Organic Chemistry 2012, 77 (13), 5577-5583.

5. Wallach, D. R.; Chisholm, J. D., Alkylation of Sulfonamides with Trichloroacetimidates under Thermal Conditions. The Journal of Organic Chemistry 2016, 81 (17), 8035-8042.

6. Harden, J. D.; Ruppel, J. V.; Gao, G.-Y.; Zhang, X. P., Cobalt-catalyzed intermolecular $\mathrm{C}-\mathrm{H}$ amination with bromamine-T as nitrene source. Chemical Communications 2007, (44), 4644-4646.

7. Wang, Z.; Zhang, Y.; Fu, H.; Jiang, Y.; Zhao, Y., Efficient Intermolecular Iron-Catalyzed Amidation of $\mathrm{C}-\mathrm{H}$ Bonds in the Presence of N-Bromosuccinimide. Organic Letters 2008, 10 (9), 1863-1866.

8. Ni, Z.; Zhang, Q.; Xiong, T.; Zheng, Y.; Li, Y.; Zhang, H.; Zhang, J.; Liu, Q., Highly Regioselective Copper-Catalyzed Benzylic C-H Amination by N-Fluorobenzenesulfonimide. Angewandte Chemie International Edition 2012, 124 (5), 1270-1273.

9. Chen, Y.; Gibson, J., A convenient synthetic route to sulfonimidamides from sulfonamides. RSC Advances 2015, 5 (6), 4171-4174. 